\title{
Abstracts of the papers presented in the international conference of Indian virological society, "Global viral epidemics: a challenging threat”, during 12-14 November, 2018, at PGIMER, Chandigarh, India
}

(C) Indian Virological Society 2019

\author{
Medical Virology \\ Complete Genome Sequencing of Measles Virus Isolates \\ Obtained during 2009-2017 from different parts \\ of India
}

Sunil R. Vaidya ${ }^{1}$, Sunitha M. Kasibhatla ${ }^{2,3}$, Divya R. Bhattad, Mukund R. Ramtirthkar ${ }^{4}$, Mohan M. Kale ${ }^{4}$, Chandrashekhar G. Raut ${ }^{5}$ and Urmila Kulkarni-Kale ${ }^{2}$

${ }^{1}$ ICMR-National Institute of Virology, Pune; ${ }^{2}$ Bioinformatics Centre, Savitribai Phule Pune University; ${ }^{3} \mathrm{HPC}-$ Medical \& Bioinformatics Applications Group, Centre for Development of Advanced Computing, Pune; ${ }^{4}$ Department of Statistics, Savitribai Phule Pune University; ${ }^{5}$ ICMR-National Institute for Research in Tribal Health, Nagpur Road, Jabalpur, India

Measles is a highly contagious viral disease of major public health concern. Measles virus $(\mathrm{MeV})$ is enveloped, single stranded negative RNA genome (15,894 nucleotides) belong to Pramyxoviridae family (genus Morbillivirus). $\mathrm{MeV}$ is serologically monotypic virus and genetically divided into 24 genotypes i.e. A, B1-B3, C1-C2, D1-D11, E, F, G1-G3 and H1-H2. World Health Organization has a target for elimination of measles and rubella control by 2020 . Government of India is committed on 'Measles Elimination' in a phased manner. Hence, a second dose of measles introduced in year 2010 through Universal Immunization Program. As per policy, suspected measles cases (fever with skin rash, cough, coryza and conjunctivitis) was monitored and laboratory confirmed by serological/molecular tests by the WHO accredited laboratory. Complete genome sequencing of thirty $\mathrm{MeV}$ isolates obtained during 2009-17 from six states of India were performed using standard RT-PCR and sequencing protocols. All these $\mathrm{MeV}$ isolates were grown on Vero hSLAM cells. Consensus $\mathrm{MeV}$ genomes/genes were subjected to phylogenetic and bioiformatic analysis. Phylogeny with global isolates revealed genotype-based spatio-temporal clustering. Estimated genome/gene-wide nucleotide substitution rates of Indian $\mathrm{MeV}$ are comparable with global isolates. Selection pressure analysis indicated modest role of positive selection in $\mathrm{MeV}$ evolution corroborating with its serologically monotypic nature. Though selection was found to be operational across all $\mathrm{MeV}$ genes except L-gene in global isolates, no positively selected sites were observed in Indian isolates. The mutation noted in the H-protein of Indian MuVs is not expected to alter the antigenicity and structural stability. Analysis of Indian and global $\mathrm{MeV}$ isolates indicates that the circulating strains during 2009-17 in India belong to genotypes D4 and D8, which have limited divergence with no potential impact on antigenicity and efficacy of the current vaccine strains in controlling $\mathrm{MeV}$ infection to meet elimination target by 2020 .

Denovo assembly of Hepatitis $\mathrm{C}$ virus full-length genome following next generation sequencing and genetic variations of strains circulating in north and north-east India

Sonu Kumar ${ }^{1}$, Renu Yadav ${ }^{1}$, Yogesh Kumar ${ }^{1}$, Chandreswar Prasad, Jyotish Kumar Jha', Kekungu Puro ${ }^{4}$, Sachin Kumar ${ }^{3}$, Anoop Saraya ${ }^{1}$, Shalimar', Suraj Nongthombam ${ }^{2}$, Baibaswata Nayak ${ }^{1}$

Email ID for Correspondence: baibaswat@gmail.com

${ }^{1}$ Department of Gastroenterology, All India Institute of Medical Sciences, Ansari Nagar, New Delhi; ${ }^{2}$ Jawaharlal Nehru Institute of Medical Sciences, Imphal; ${ }^{3}$ Department of Biosciences and Bioengineering, IIT Guwahati; ${ }^{4}$ ICAR Research Complex for NEH Region, Meghalaya

Introduction: Hepatitis $\mathrm{C}$ virus (HCV) infection is silent and infected patient develop chronic hepatitis with increased risk of cirrhosis, hepatocellular carcinoma and end stage live disease. Current treatment with direct acting antivirals (DAAs) is very effective in achieving sustained virological response (SVR). However, expected drug resistance and suboptimal activity against diverse HCV genotypes are major drawback. Next generation sequencing (NGS) with more coverage will be effective in studying genetic variations in both wild type and resistance associated variants (RAV) and immune response. HCV genotyping by commercial real time PCR assays escapes subtypic variations within genotype that need to be evaluated by direct sequencing for unusual subtype that may evade SVR. The serum samples from treatment naïve consecutive chronic hepatitis $C$ patients at AIIMS (North India) and JNIMS, Imphal were obtained to study genetic variation of HCV. Real time PCR for HCV quantitation and genotyping was carried out. The next generation sequencing and NS5b region Sanger sequencing of HCV genome was carried out 
Denovo assembly and phylogenetic analysis of strains were done using web based bioinformatics software.

Out of $90 \mathrm{HCV}$ samples collected at AIIMS, Genotype 1 was $21 \%$ and genotype $3 \mathrm{a}$ was $78 \%(\mathrm{n}=71)$ whereas among 142 north east sample, genotype 1 was $29 \%(n=42), 3$ was $60.5 \%(n=86), 4$ was $1.5 \%(\mathrm{n}=2)$ and genotype 6 was $8.5 \%(\mathrm{n}=12)$. HCV complete genome cDNA and library preparation was carried out for NGS. The sequence data were subjected for filtration and exclusion of human sequence by mapping with HG19 sequence. The remaining reads were used for denovo genome assembly. Three north-east strains from genotype 1 and 3 were sequenced and analyzed. Phylogenetic analysis of NS5b region of north Indian HCV strain have shown $3 \mathrm{a}, 3 \mathrm{~b}, 3 \mathrm{~g}$ and $3 \mathrm{i}$ subtypes within genotype $3 \mathrm{HCV}$.

\section{Delineating the role of DNA damage response during Rotavirus replication}

\section{Sarkar R, Chawla-Sarkar M}

Email ID for Correspondence: chawlam70@gmail.com Division of Virology, National Institute of Cholera and Enteric Diseases, Kolkata, India

Cellular DNA damage response (DDR) machinery is present in all eukaryotic cells to monitor and repair DNA in response to cellular DNA damage. Induction of cellular DDR results in the activation of cell cycle arrest, DNA repair pathway or apoptosis. Several studies have shown that both DNA and RNA viruses can activate the cellular DDR pathway and hijack this pathway to facilate their own replication. This study was conducted to check the activation of DDR following Rotavirus infection and find out its role in Rotavirus replication. Western blotting was performed to check the expressions of cellular and viral proteins following Rotavirus infection. Expressions of viral RNAs were quantified by qRT-PCR. Cellular DNA damage was measured by comet assay. Localization of cellular proteins were visualized by immunofluorescence technique. Infective virion particles formation was measured by plaque assay. Results have shown that Rotavirus infection induces the activation of the ATM branch of DDR pathway. ATM activation was found to be independent of cellular DNA damagebut dependent on double stranded break sensor MRN complex as knockdown of MRE11 and RAD50 by lentivirus prevented ATM activation. Activated form of ATM, CHK2 and MRN complex components, MRE11 and RAD50 were found to localize within the viroplasm. Inhibition of ATM and CHK2 proteins by chemical inhibitors block viral replication as observed by reduced viral protein synthesis, viral RNA synthesis and viral titers, suggesting a crucial role of ATM protein in viral replication. Overall, these results suggest that rotavirus infection induces the activation of ATM branch of cellular DDR and exploit this pathway for its own replication.

\section{Characterization of Human papillomavirus type 16 major capsid protein variants as markers of cervical neoplastic status with functional and phylogenetic analysis}

\author{
Mane $\mathbf{A}^{1}$, Patil $\mathbf{L}^{1}$, Limaye $S^{2}$, Kulkarni-Kale $\mathbf{U}^{2}$ \\ ${ }^{1}$ ICMR-National AIDS Research Institute, Bhosari, Pune, India; \\ ${ }^{2}$ Savitribai Pune University, Ganeshkhind Road, Pune, India
}

The etiological role of infection with high-risk Human papillomavirus (HPV) types and cervical cancer is well established, while HPV type 16 is most prevalent globally and in India. In a given HPV type, the genome is further classified into 'variants' if presence of nucleotide variations is $<2 \%$ in coding regions. Major capsid or L1 protein is corner stone in HPV structure. L1 variants can affect viral assembly and restrict immunological recognition. Developing information on $\mathrm{L} 1$ variants is imperative for rational design of newer diagnostics and vaccine. The present study was carried out to characterize HPV16 L1 variants by cervical intraepithelial neoplastic status, evaluate their impact on epitopes and determine their phylogeny. HPV16 L1 open reading frame was analyzed in 152 well characterized archival HPV16 positive cervical samples (48 with normal, 51 with low-grade and 53 with high-grade cervical status). Intratype variants were analyzed using PCR-directed sequencing, impact on experimentally validated epitopes was predicted in silico, while phylogenetic analysis was carried out using MEGA software with maximum likelihood method. A total of 58 nucleotide polymorphisms were detected, of which 26 were novel and 20 non-synonymous. Commonest L1 variants were A6432G (73.7\%), G7058T (17.8\%) and C6852T (16.4\%). L1 variants C5862T (H102Y), C6163 (T202N), A6178C (N207T), A6693C (T379P) and A6801T (T415S) showed significant association with high-grade cervical disease and an increasing trend with cervical disease progression. Predominance of European lineage $(84.2 \%)$ was observed, while non-European lineages were associated with high grade cervical intraepithelial neoplasia, $p=0.012$. Ten variants were part of experimentally validated $\mathrm{B}$ cell and three of $\mathrm{T}$ cell epitopes respectively. Five variants were located in the immunogenetic FG and HI capsid loops and could potentially impact antiHPV vaccine efficacy. Results suggest association of specific L1 variants and non-European lineages with cervical intraepithelial neoplasia.

\section{Development and evaluation of rapid field compatible sample to answer detection technique for rapid on-site detection of Chikungunya virus}

\section{Shashi Sharma ${ }^{1}$, Paban Kumar Dash' ${ }^{1}$, M.M.Parida ${ }^{1}$, Deepak Pardasani ${ }^{2}$, D.K. Dubey ${ }^{2}$}

Virology Division ${ }^{1}$ DRDE, Gwalior,Vertox Division ${ }^{2}$ DRDE, Gwalior,Defence food research laboratory, Mysore

Chikungunya virus has re-emerged as an important pathogen causing epidemics in several parts of the world since 2004. Predominantly, CHIKV is often associated with severe persistent arthralgia in small joints. The confirmed circulation of CHIKV in India, and South-East Asia is a serious concern and cases from all parts of the country are reported throughout the year. The rapid molecular detection is required for effective control measure against this viral infection, as the virus share similar symptoms so often misdiagnosed with Dengue and Zika virus infection. The current limitation in existing rapid detection assay is the lack of suitable rapid field compatible sample extraction platform. We report standardization of inhouse magnetic bead based nucleic acid extraction method and reverse Transcription Polymerase spiral reaction (RT-PSR), an innovative isothermal amplification technique for rapid detection of CHIKV. Subsequently both the methods were validated using clinical samples. In addition, dry down RT-PSR reagents were prepared and successfully evaluated.

The in-house magnetic bead based nucleic acid extraction followed by dry down RT-PSR assay was found to be highly sensitive and specific. The sensitivity was also compared with commercially available viral RNA extraction kit and real time RT-PCR. The detection limit of dry down RT-PSR was found to be 10 copies of 
RNA transcript. This novel methodology will help in developing a rapid, field compatible on-site detection platform as a concept for viral agents.

\section{One-step single tube accelerated quantitative nucleoprotein gene specific reverse transcription loop}

irradiation. Different UV doses were used to inactivate the CHIKV. The replication status of the inactivated virus was verified in cell lines. Western blot, electron microscopy (EM) and Immune fluorescence assay were used respectively to see the antigenicity, structural integrity and entry of the virus into cell lines. The inactivation was complete when a UV dose of $0.09 \mathrm{~J} / \mathrm{cm} 2$ for $3 \times 30 \mathrm{~s}$ was used and no change in antigenicity and integrity observed. The study concludes that the virus is antigenically stable and could be used in BSL-2 settings for different experiments.

\section{Immune Modulation of Macrophages during Chikungunya Virus infection}

Ebola virus (EBOV) causes severe hemorrhagic fever in humans and nonhuman primates with high mortality rates. The Centres for Disease Control and Prevention has classified them as "Category A Bioterrorism Agents" as they could be easily transmitted, result in high mortality, cause major public health impact and panic, and require special action for public health preparedness. The recent outbreak of Ebola virus in West Africa highlights the urgent need to develop a rapid and real-time detection system for early clinical diagnosis for better patient management. Isothermal amplification of nucleic acids has emerged as a promising alternative in which rapid and efficient amplification is achieved at a constant temperature without the thermal cycling required in PCR. A one-step single tube accelerated quantitative reverse transcription loop mediated isothermal gene amplification (RTLAMP) assay was developed by targeting the nucleoprotein (NP) gene of Ebola virus. The RTLAMP assay was found to be specific for Ebola virus, without having any cross reactivity with related haemorrhagic fever viral agents. The comparative evaluation of Ebola virus NP gene specific RTLAMP assay with RTPCR and Taqman real-time RT-PCR demonstrated that RTLAMP was $10-1000$ fold more sensitive than Taqman real-time RT-PCR and conventional RTPCR respectively with a detection limit of 1 copy number. Ebola virus RTLAMP assay for clinical diagnosis was evaluated with different kind of spiked body fluids including serum, urine, saliva, semen and stool samples of healthy human volunteers. The Ebola virus RTLAMP assay could correctly picked up the spiked samples up to 1 copy of viral RNA without having any matrix interference. The monitoring of gene amplification can also be visualized with the naked eye by using SYBR green I fluorescent dye. Thus, due to easy operation without a requirement of sophisticated equipment and skilled personnel, the RT-LAMP assay reported here is a valuable tool as a point of care diagnostic for the rapid and realtime detection of Ebola virus in resource limited health care settings of developing countries.

\section{Inactivation of Chikungunya virus by Ultraviolet Light}

\section{Asha Maria Mathew ${ }^{1}$, Amol Baburao Mun', Anukumar Balakrishnan ${ }^{1}$}

Email ID for Correspondence: mathewashamaria@gmail.com, anukumar74@gmail.com

National Institute of Virology ${ }^{1}$, Kerala Unit, T.D. Medical College Hospital, Vandanam, Alappuzha ${ }^{2}$, Kerala, India

Chikungunya virus (CHIKV) is a rapidly emerging arbovirus causing millions of infections in more than 40 countries. CHIKV is typically a Biosafety level 3 (BSL-3) pathogen in many countries and handling of CHIKV requires a high standard laboratory safety settings. Many studies require the whole virus and the virus to be handled in Biosafety level-2 setting. A potential solution for managing this problem is pathogen inactivation without affecting its antigenicity. In the present study, we attempted to inactivate the CHIKV by UV
Tapas K. Nayak' ${ }^{1}$, Prabhudutta Mamidi ${ }^{2}$, P Sanjai Kumar1, Subhransu S. Sahoo' ${ }^{1}$, Chandan Mahish ${ }^{1}$, Soma Chattopadhyay ${ }^{2}$, and Subhasis Chattopadhyay ${ }^{1}$

Email ID for Correspondence: subho@niser.ac.in

School of Biological Sciences, National Institute of Science

Education \& Research, Bhubaneswar ${ }^{1}$, HBNI, Jatni, Khurda, Odisha, Infectious Disease Biology, Institute of Life Sciences, (Autonomous Institute of Department of Biotechnology, Government of India), Nalco Square ${ }^{2}$, Bhubaneswar, Odisha, India

Re-emergence of Chikungunya virus (CHIKV) infection has referred as a major public health concern due to its recent worldwide epidemics and lack of treatment measures. Even though macrophages are known to harbour CHIKV infection, the concerned regulatory mechanism of the viral replication and the related immune responses are not well understood. Raw264.7 cells (mouse macrophage cell viral progeny release were assessed by flow cytometry and plaque assay, respectively. Furthermore, immune modulation of host macrophages and apoptosis were studied through Flow cytometry, Western blot and ELISA. Our current observations suggest that expression of CHIKV proteins were maximum at $8 \mathrm{hpi}$ and the release Macrophages undergo both extrinsic and intrinsic pathways of apoptosis. The pro-inflammatory mediators, MHCs and B7 were also found to be augmented during infection in indicating robust macrophage immune activation. Moreover, it was also observed that the MAPKs are activated during CHIKV infection and associated with TNF production in the host macrophages in $\mathrm{p}-\mathrm{c}-\mathrm{JUN}$ dependent pathway. Further, 17-AAG (a potential HSP90 inhibitor) was found to regulate CHIKV infection, apoptosis and pro-inflammatory cytokine/ chemokine productions of host macrophages significantly.

\section{Evaluation and determination of diagnostic performance of quantitative HBsAg in Chronic Hepatitis B virus infected patients of Bangladesh}

\section{S M Rashed UI Islam, Munira Jahan, Shahina Tabassum}

Email ID for Correspondence: smrashed@bsmmu.edu.bd, smrashed1620@yahoo.com

Department of Virology, Bangabandhu Sheikh Mujib Medical University (BSMMU), Dhaka, Bangladesh

Hepatitis B Virus DNA (HBV-DNA) viral load measurements are recommended for diagnosis and monitoring of patients on the treatment of Chronic Hepatitis B (CHB). In addition, quantitative $\mathrm{HBsAg}$ (qHBsAg) estimation adjunct to HBV-DNA is vital to assess the state line) were infected with CHIKV and viral replications as well as new of new viral progenies were significantly increased around $12 \mathrm{hpi}$. 
of HBV chronicity and therapeutic prognosis. Therefore, this study was aimed to estimate the qHBsAg and to compare its diagnostic performance with the HBV-DNA level in CHB patients at tertiary care set up. A total of $148 \mathrm{CHB}$ virus-infected patients were enrolled in this study. qHBsAg and $\mathrm{HBeAg}$ were performed using Chemiluminescent (LIAISON ${ }^{\circledR} \mathrm{XL}$ ) and Enzyme Immunoassay respectively and HBV-DNA were done using Real-time PCR (Applied Biosystems $\left.{ }^{\circledR} 7500\right)$. Comparisons among the groups and diagnostic performance (Receiver operating characteristic; ROC) were analyzed with SPSS v21. The median $\mathrm{qHBsAg}$ of the study population was $4.35 \times 10^{3} \mathrm{IU} / \mathrm{ml}$ and $45.9 \%$ were $\mathrm{HBeAg}$ seropositive. Out of the total $148 \mathrm{CHB}$ patients, $86.5 \%$ were found with detected HBV-DNA $\left(>5 \times 10^{2} \mathrm{IU} / \mathrm{ml}\right)$ with the median viral load of $1.91 \times 10^{4} \mathrm{IU} / \mathrm{ml}$. Among the patients with Undetected HBV-DNA $\left(<5 \times 10^{2} \mathrm{IU} / \mathrm{ml}\right)$, median qHBsAg was $2.05 \times 10^{3} \mathrm{IU} / \mathrm{ml}$. Significant differences were observed in both qHBsAg $(p<0.001)$ and HBV-DNA $(p<0.0001)$ according to $\mathrm{HBeAg}$ sero-positivity. Correlation analysis between qHBsAg and HBV-DNA revealed positive correlation $(\mathrm{r}=0.30$; $p<0.0001)$. ROC curve analysis showed, the area under the curve (AUC) for the qHBsAg level to distinguish high HBV-DNA $\left(>1 \times 10^{5} \mathrm{IU} / \mathrm{ml}\right)$ was $0.65(p=0.002)$ highlighting good diagnostic performance. The best cut-off of qHBsAg for predicting high HBVDNA level was $2.95 \times 10^{3} \mathrm{IU} / \mathrm{ml}$. This study is the first of its kind to quantitate HBsAg in $\mathrm{CHB}$ patients from Bangladesh. Use of this marker may bring about an important complementary evaluation to HBV-DNA among CHB patients during treatment and follow-up.

\section{Analysis of microRNA expression in Chronic Hepatitis C Virus Infected Patients in Bangladesh}

\section{Hossain Sa, Islam SMRb, Jahan Mb, Tabassum Sb, Yasmin Ma}

University of Dhaka (DU) Bangabandhu Sheikh Mujib Medical University (BSMMU), Bangladesh

MicroRNAs (miRNAs) belong to a class of non-coding RNAs that are secreted by the cells into the circulation. Aberrant circulatory miRNAs profile are associated with different infectious diseases including HCV-related liver diseases. This study has been designed to explore the miRNA profile among chronic HCV patients in Bangladesh with the goal of finding suitable candidate biomarkers. Expression levels of four miRNAs (hsa-miR-122, hsa-miR-92a, hsa- miR-200b and hsamiR-21) in serum samples from thirty-nine patients with HCV viremia and twenty healthy volunteers were analyzed using Real-time PCR. The relative expression level of miRNA and diagnostic performance of selected miRNAs were performed with SPSSv21. In addition, miRNA induced target prediction and pathway analysis were performed using miRSystem and Enrichr web based bioinformatics tools respectively. Relative expression analysis revealed that all the miRNAs except hsa-miR-21 were up-regulated $(P=0.002$ for hsa-miR-122 and $P<0.0001$ for both hsa-miR-92a and hsa-miR$200 \mathrm{~b}$ ) in the sera of HCV patients compared with that in healthy controls. Furthermore, the receiver operating characteristic curves showed that hsa-miR-122 (Area under curve $(\mathrm{AUC})=0.73$, $P=0.002)$, hsa-miR-92a (AUC $=0.95, P<0.0001)$, hsa- miR-200b (AUC $=0.88, P<0.0001)$ could distinguish HCV patients with desirable sensitivity and specificity. In addition, correlation analysis indicated serum hsa-miR-92a expression was positively correlated ( $\mathrm{r}=0.41, P=0.01$ ) with HCV viral load. Pathway analysis of putative target genes of hsa-miR-92a indicated the involvement of TGF- $\beta$ signal transduction, HIF-1 signaling, ECM-receptor interaction and mTOR signaling pathways indicating their role in $\mathrm{HCV}$ replication, chronic HCV mediated fibrogenesis and growth dysregulation of cells. This study revealed that, these miRNAs, hsa-miR-92a, in particular, are aberrantly expressed in chronic HCV patients and have the potential to serve as non-invasive biomarkers for HCV infection. Further functional analysis highlighted the possible association of host miRNAs targeted gene regulation during the course of $\mathrm{HCV}$ infection.

\section{Identification and characterization of potential dengue virus receptors in Aedes aegypti}

\section{Yadav K, Rana S V, Rajagopal R}

Email ID for Correspondence: karunayadavmh1993@gmail.com Gut Biology Laboratory, Department of Zoology University of Delhi110007, India

Aedes aegypti is a major insect vector for dengue virus transmission across the world. Dengue virus symptoms involve mild fever to haemorrhagic disorders in humans as well as non-human primates. The number of dengue disease incidences have increased dramaticallyover the past decade. Till date, no universal vaccine is available against all four serotypes, hence controlling the population of vector is the key strategy for regulating the transmission of dengue virus to humans. The molecular understanding of relationship of virus and vector is critical to design the vector based strategy for controlling the dengue epidemic. A cDNA library derived from the pool of A. aegypti mosquito was established using SMART (switching mechanism at $5^{\prime}$ end of RNA Transcript) in the combination of welldeveloped and efficient molecular machinery of Saccharomyces cerevisiae. Yeast two-hybrid (Y2H) assays were performed using DENV-2 Envelope protein as a bait for identifying the potential interacting proteins. Yeast clones growing on 3DO, 4DO and exhibiting the expression of alpha-gal assay were selected and sequenced. Sequencing data was searched for similarity on NCBI database. A number of potential mosquito proteins that interact with DENV-2 proteins were identified. Protein like apolipoprotein D, phosphatase were identified. The identified interacting proteins might involve in different biological activities that may assist in replication of virus inside the insect vector. Identification of different interacting proteins of dengue virus in the insect vector host is an crucial aspect for the understanding of its transmission process which can further be useful in designing vector based prevention strategies.

\section{A multi-centric hospital based study on epidemiology of keratoconjunctivitis in India}

\section{Email ID for Correspondence: rahuldhodapkar@gmail.com}

Viral keratoconjunctivitis which is the most commonly observed ocular conditions in clinical practice. Though self-limiting it is associated with morbidity, loss of man working hours and productivity. Most of the studies/surveillance programs for conjunctivitis are targeted towards one or more pathogens. There is paucity of published literature spanning entire viral spectrum causing viral conjunctivitis especially in India, most of the work carried out on keratoconjunctivitis is limited to outbreak investigation and characterization. This study was designed to conduct a nation-wide screening for the most common causes of viral conjunctivitis. For this purpose we had identified 4 centres PGIMER, Chandigarh; TNMC \&BYL NAIR HOSPITAL, Mumbai; NEIGHRIMS, Shillong; RMRC, Port Blair along with our centre JIPMER, Puducherry. The most common 
causative agent for viral keratoconjunctivitis includes Adenoviruses, Enteroviruses, Herpes Simplex viruses (HSV) in their descending order of disease causing frequency and Chlamydia. Adenoviruses (serotypes 3, 4, 7, 8, $19 \& 37$ ) are one of the most common causative agents for keratoconjunctivitis worldwide with recent outbreaks caused by Adenovirus serotype 8. Enteroviruses (EV71 and Coxsackie A24) which are known to cause CNS infections are also associated with haemorrhagic conjunctivitis. Herpes simplex virus, unlike the other two viruses does not cause outbreaks. It is primarily responsible for herpetic corneal involvement classically presenting as geographical ulcers. Chlamydial ocular involvement presents in form of Trachoma and Inclusion conjunctivitis which at times may be indistinguishable from viral and hence was included in the current study. At the end of first year a total of 452 samples have been collected from all the centres, of which the positives were Adenovirus - 154, Enterovirus - 11 and HSV - 7. Preliminary analysis shows that the major causative agent is Adenovirus (34\%), which when genotyped showed mostly genotype 8 .

\section{Role of poly A tail in hepatitis $\mathbf{E}$ virus replication efficiency}

\section{Bhise NA, Mustak SS, Lole KS}

\section{Email ID for Correspondence: bhisena@gmail.com}

Hepatitis E virus (HEV) is an important public-health concern and major cause of enterically transmitted hepatitis worldwide. HEV is a positive sense RNA genome with 7-methylguanosine cap at $5^{\prime}$-end and a poly (A) tail at $3^{\prime}$-end, lacking efficient cell culture system in spite of several attempts to adapt the virus. Present study aims to see the influence of poly A tail on HEV replication. HEV subgenomic replicon plasmid harbouring Renilla luciferase gene (pSK HEV-2 Rluc) with poly A tail of 16 A residues was used as template. Primers were designed to introduce deletions or insertions in poly A tail of the construct by site directed mutagenesis. Two deletion mutants were developed by removing 3- and 6-A residues from the (mut13: with 13-A residues and mut10: with 10-A residues) respectively and mutants were produced by adding 3 and 6-A residues to construct (mut19: with 19-A residues and mut22: with 22-A residues) to the original replicon. Plasmid constructs were used as templates to generate HEV RNA replicons by in vitro transcription and transfected into human hepatoma cells (S10-3). Replication of HEV was monitored by dual luciferase reporter assay. Sixfold reduction in replication of mut $13 \mathrm{HEV}$ replicon and nearly complete inhibition of replication of mut10 replicon was observed, indicating the requirement of at least 13-A residues in poly A tail for successful virus replication. Extension of poly A tail by 3- and 6- A residues in mut 19 and mut 22 resulted in twofold and threefold enhancement respectively in virus replication as compared to original replicon. Poly (A) tail length plays a very significant role in the HEV replication efficiency.
Screening for high risk Human Papilloma Virus (HPV) genotyping by multiplex Real Time PCR using TOCE technology in womens with cervical abnormalities: a tertiary hospital based study from Raipur, Chhattisgarh

\section{Negi SS*, Bhargava A, Singh P, Hussain N, Aggarwal S}

Department of Microbiology, AIIMS, Raipur, Chhattisgarh, India

Cervical cancer is the fourth most common malignancy in females worldwide. The screening of human papillomavirus (HPV) by molecular tests holds promise for reducing cervical cancer incidence and mortality in low resource countries like India. Accordingly in this study high risk (HR) HPV genotyping was evaluated against cytology and colposcopy for primary cervical screening in the womens with cervical abnormalities. A total 185 clinical sample of cervical scrapes obtained from womens attending gynecology department for routine cervical examination were processed by multiplex Real-time PCR using TOCE technology to target L1 gene to detect and identify the 14 HR-HPV genotype and compared with cytology and colposcopy examination. Among 185 samples, detection rate of HPV by RT-PCR was found $36.2 \%$ (67 cases) versus Colposcopy $21.08 \%$ (39 cases) and cytology $22.16 \%$ (41 cases) $(p<0.01)$. HPV 16 was found most prevalent with detection in 18 cases $(8.6 \%)$ followed by HPV 39 (7 cases, 3.78\%), HPV 18 (06 cases, 3.24\%), HPV 31 (04 cases, 2.16\%), 68(03 cases, $1.62 \%)$, HPV 59 ( 2 cases, $1.08 \%$ ), and HPV 52 (01 case, $0.54 \%) .26$ cases $(14.05 \%)$ were found with more than one HR HPV genotypes. PAP smear examination showed ASCUS in 26 cases (14.05\%), LSIL in 08 cases $(4.32 \%)$, HSIL in 02 cases $(1.08 \%)$, SCC in five cases $(2.70 \%)$. HPV genotyping with high detection rate over other conventional procedures may be used in routine cervical screening for higher probability of detection of HR-HPV genotypes to allow rapid and pragmatic subsequent management to prevent morbidity and mortality. HPV genotyping also provided the information of unusual higher prevalence of HPV genotype 39 in comparison to HPV18. The baseline data of prevalence of HR-HPV may help vaccine manufacturer to consider these genotypes before preparing future polyvalent vaccines.

\section{Recombinant adeno-associated virus mediated effective inhibition of human metapneumovirus in mammalian cells}

Prashant Kumar, Unnati Singh, Mansi Srivastava

Amity Institute of Virology \& Immunology, Amity University Noida Uttar Pradesh

Development of universal vaccines against human metapneumovirus (HMPV), a leading cause of respiratory infection, has been a challenge due to lack of long term memory in the infected/immunized individual. There is an urgent need for an alternative prophylactic/ therapeutic agent which may restrict the virus infection in human cells. Antisense oligonucleotides have been shown to be effective in down-regulating different virus replications in various models but lack of suitable delivery systems and toxicity due to constitutive expression limits their use for therapeutic/prophylactic purposes. We aimed to develop an adeno-associated virus based delivery system for induced expression of HMPV specific shRNA and ribozymes in target human cells. We developed chimeric constructs encoding shRNAs and ribozymes specific for human metapneumovirus genome and studied their efficacy and expression profiles in HEK293 cells. A 
recombinant adeno-associated virus (rAAV5), having a modified genome expressing the chimeric construct, was generated and its capacity to introduce the construct in human cells as an episome or integrated part of human chromosome was studied. We further investigated the ability of rAAV infected human cells to restrict the hMPV infection. Out of five shRNAs and five ribozymes designed to target the hMPV genome, two shRNAs and one ribozyme was effective in down-regulating the viral proteins in HEK293 cells. The shRNA-ribozyme chimeric construct could show the additive effect of shRNA and ribozyme in down-regulating the viral proteins. rAAV particles expressing the chimeric construct could infect the human cells and establish the episome in infected cells which could further express the shRNAs and ribozymes in these cells. These shRNAs and ribozymes could significantly reduce the hMPV load in rAAV infected cells. We concluded that rAAV particles could serve as a potent delivery vehicle for therapeutic nucleic acids to restrict the propagation of HMPV in human cells.

\section{Exploring rotavirus proteins to design B and T-cell multi-epitope vaccine using in silico approach}

\section{Damayanti Yengkhom, Arpita Devi, R. Doley, Nima D. Namsa}

Email ID for Correspondence: namsa@tezu.ernet.in Department of Molecular Biology and Biotechnology, School of Sciences, Tezpur University, Assam, India

Rotavirus (RV), a dsRNA virus in the family Reoviridae, is the leading cause of severe acute gastroenteritis in infants and young children of $<5$ years of age. The genome consists of 11 dsRNA segments which codes for six structural (VP1, VP2, VP3, VP4, VP6 and VP7) and six non-structural proteins (NSP1, NSP2, NSP3, NSP4, NSP5 and NSP6). Each viral protein has a specific role in viral infection and pathogenesis. There has been less extensive study of primary $\mathrm{T}$ - or $\mathrm{B}$-cell responsiveness to rotavirus infection and the functional role of these cells or their correlation to long term immune protection. In this study, a comprehensive immuno-informatics approach was applied to map the presence of immunodominant B and T-cell epitopes of rotavirus structural and nonstructural proteins. A multi-epitope based vaccine constructs was designed encompassing the predicted B and T-cell epitopes that were selected based on flexibility and accessibility. The allergenicity and antigenicity were predicted to ensure the safety and immunogenic behavior of final vaccine constructs. The physiochemical parameters such as theoretical pI, molecular weight, aliphatic index and GRAVY score was also calculated. Further, multiple-threading modeling followed by molecular dynamics simulation confirms the overall stability of the final vaccine constructs and codon optimization was performed to improve the expression of recombinant multi-epitope antigens in bacteria. The findings of this study may implicate in developing effective epitope-based vaccine in controlling rotavirus infection and diagnosis of rotavirus in epidemiological surveillance.

\section{Discovery and validation of Chikungunya antivirals targeting viral protease}

\section{Tomar S, Singh H, Mudgal R, Fatma B, Kumar R}

Email ID for Correspondence: shailfbt@iitr.ac.in Molecular Virology Laboratory, Department of Biotechnology ${ }^{1}$, Indian Institute of Technology Roorkee, Uttarakhand, India
Chikungunya virus (CHIKV), a mosquito born pathogenic virus, causes fever, arthritis and encephalitis in humans. Presently, there is no specific drug/antiviral available for CHIKV treatment. Upon entry into host cell, ssRNA of CHIKV is directly translated into nonstructural polyprotein precursor $\mathrm{P} 1234$ which is processed by virus specific nsP2pro. This nsP2pro mediated processing is an essential function in viral life cycle and thus nsP2pro constitutes an attractive target for antiviral drug development.

In this study, we have elucidated the $3 \mathrm{D}$ structure of CHIKV nsP2pro which revealed the presence of two subdomains; N-terminal protease subdomain and a C-terminal MTase-like subdomain. Active site of the protein consists of a catalytic dyad made up of cysteine and histidine and is located at the interface of the two subdomains. Detail structural analysis and comparison revealed the presence of a flexible loop gating the active site. This loop possesses conserved residues Asn547 and Trp549. Furthermore, by mimicking the intermolecular interaction between substrate peptide and the active site residues of nsP2pro, a series of peptidomimetic compounds were identified. The conformational stability of these compounds was assessed by molecular docking and MD simulation. We developed a FRET based nsP2 protease activity assay to assess the inhibitory potential of identified compounds. Interestingly, two of the identified peptidomimetic compounds were found to possess inhibitory effect on the nsP2pro enzymatic activity. Inhibition of CHIKV nsP2pro by the tested compounds Pep-I \& Pep-II was observed with IC50 values of $34 \mu \mathrm{M}$ and $42 \mu \mathrm{M}$, respectively. The inhibition kinetic studies revealed the inhibition constant $(\mathrm{Ki})$ to be $33.34 \pm 2.53 \mu \mathrm{M}$ for Pep-I and $45.89 \pm 4.38 \mu \mathrm{M}$ for Pep-II. Additionally, these two compounds were also validated by cell culture based plaque reduction assay and found to be significantly inhibiting CHIKV replication in BHK-21 cells at concentrations in $\mathrm{nM}$ range, which is much lower than their cytotoxic concentrations.

\section{Identification and characterization of differentially expressed host proteins modulated during Chikungunya virus infection}

\author{
Mamidi $\mathbf{P}^{\mathbf{1}}$, Nayak $\mathbf{T K}^{\mathbf{2}}$, Chatterjee $S^{\mathbf{1}}$, De $S^{\mathbf{1}}$, Datey $A^{\mathbf{1}}$, \\ Chattopadhyay $S^{2}$ and Chattopadhyay $S^{1 *}$
}

${ }^{1}$ Institute of Life Sciences, Bhubaneswar; ${ }^{2}$ School of Biological Sciences, National Institute of Science Education \& Research, Bhubaneswar, India

The emergence of Chikungunya virus (CHIKV) as a global pathogen emphasizes the need to understand the biology of this virus for the development of effective control measures. Earlier, it was reported by us that Indian outbreak strain (DRDE-06) exhibits faster replication than CHIKV prototype strain S 27 in vitro. Therefore, a comparative study was carried out between S 27 and DRDE-06 for identifying the host factors that are modulated during CHIKV infection. Microarray analysis was carried out for S 27 and DRDE-06 infected Vero cells to understand the modulation of host genes. Out of the many, few selected genes were studied further to explore their functional significance in CHIKV infection. Analysis of microarray data revealed that stress response, translational control and MAPK pathways related genes were significantly modulated during CHIKV infection. Out of which, MAP kinase activated protein kinase 3 (MK3) and its isozyme partner, MAP kinase activated protein kinase 2 (MK2), belonging to p38MAPK pathway were chosen for further analysis. Both, simultaneous gene silencing of MK2/MK3 through siRNA as well as CMPD1 (MK2a specific inhibitor) treatment reduced CHIKV progeny release significantly in vitro with respect to control. Furthermore, CMPD1 treatment, in CHIKV infected suckling C57BL/6 mice 
reduced both CHIKV RNA expression as well as expression of inflammatory cytokines in the serum and showed $100 \%$ survival in comparison to untreated mice. MK2 and MK3 were identified as novel host factors involved in CHIKV infection inside the host system which can be targeted to modulate the disease caused by CHIKV.

\section{The Association of BAX-248G $>$ A and BCL2- 938C $>$ A Polymorphism with the Risk of Epstein Barr virus associated Nasopharyngeal Carcinoma: Prediction of Biomarkers for the Detection of the Disease in North-Eastern Indian Population}

\author{
Chatterjee Koustav ${ }^{1}$, De $S^{2}$, Sahu $S^{1}$, Das $\mathbf{P}^{1}$, \\ Chattopadhyay NR ${ }^{1}$, Choudhuri $T^{1}$
}

Department of Biotechnology, Visva-Bharati, Santiniketan, ${ }^{1,2}$ WB, India, Institute of Life Science, NALCO Square, Bhubaneswar, Odisha, India

Pro-apoptotic BAX and anti-apoptotic BCL2 are two key proteins in the BCL2 family which are involved in apoptotic regulation. Single nucleotide polymorphisms (SNP) in the promoter region of BAX and BCL2 are reported to have altered expressions in many cancers. Here we investigated the association of BAX-248G > A and BCL2938C > A polymorphism with the prognosis of EBV associated Nasopharyngeal Carcinoma (NPC) in the North-Eastern Indian population. To detect the presence of EBV, PCR was done. PCR-RFLP and DNA sequencing were performed for the genotyping to detect polymorphism from both NPC samples and Healthy Controls, collected from different State Hospitals in North-Eastern India. Insilico study was conducted to inspect any alteration of transcription factors'(TFs) binding due to SNP. We also conducted meta-analysis to study the consequence of these SNP with the risk of the overall cancer susceptibility. We have found the presence of EBV in NPC samples. From the genotype and allelic distribution, we observed an increased risk of NPC in BAX -248 GA $(P=0.009)$, combined GA-AA genotype $(P=0.005)$ and A allele $(P=0.003)$. BCL2-938 AA $(P=0.03)$, combined CA-AA genotype $(P=0.02)$ and A allele $(P=0.004)$ have shown significant association with the increased risk of NPC. From the insilico approach, the BAX promoter polymorphisms have shown the reduced and unstable binding affinity for $\mathrm{p} 53$ and sp1 TFs towards the promoter. An increased binding affinity of STAB 1 and cyclic AMP response element (CRE) has observed due to BCL2 polymorphism. In meta-analysis study, besides some ethnic model no such significant association have found between BAX248G $>$ A or BCL2-938C $>$ A SNP and the overall cancer susceptibility. We concluded that promoter polymorphisms of BAX $248 \mathrm{G}>\mathrm{A}$ and BCL2 -938C > A could be used as a biomarker for the detection of EBV associated NPC in North-Eastern India.

\section{Mutational and structural Insights into West Nile virus RNA dependent RNA polymerase for antiviral discovery}

\author{
Kumar $\mathbf{R}^{\mathbf{1}}$, Rani $\mathbf{R}^{1}$, Kuhn $\mathbf{R} \mathbf{J}^{\mathbf{2}}$, Tomar $\mathbf{S}^{1, *}$ \\ Email ID for Correspondence: shailfbt@iitr.ac.in \\ ${ }^{1}$ Molecular Virology Laboratory, Department of Biotechnology, \\ Indian Institute of Technology Roorkee, Roorkee, Uttarakhand; \\ ${ }^{2}$ Markey Center for Structural Biology, Purdue Institute of
}

Inflammation, Immunology and Infectious Disease Department of Biological Sciences, Purdue University, Indiana

West Nile virus (WNV), a member of the Flaviviridae, is a positivesense single-stranded RNA virus. The replication of viral-RNA genome in flaviviruses is dependent of viral-RNA polymerase. Mutational analysis and residue swapping have been done to clarify the role of the Nuclear-Localization-Sequence residues in RNA-dependent-RNA-polymerase (RdRp) activity. Expression and purification of NS5 mutants of WNV-RdRp active-domain with wildtype were done using prokaryotic-expression-system and Hi-Trapnickel-immobilized-metal-ion-affinity-chromatography. The inactiveRdRp-mutant was made by site-directed-mutagenesis to substitute the active site GDD-motif with GAA using the QuikChange-mutagenesis protocol and confirmed by DNA-sequencing. Expression and purification of inactive-RdRp-mutant were also done. RdRp activity assay was assessed by the primer-extension assay. The reaction product was resolved in denaturing-RNA-PAGE and the bands were visualized using Typhoon-FLA-900scanner. The intensities of the observed bands were quantified using Quantity-one-1D analysis software. Further, to identify potential flavivirus NS5 inhibitors, in silico screening and molecular docking was done and three potential molecules have been identified. The RdRp assay was done for all five constructs with the optimal concentration of all required reagents, incubation temperature and time. Based on the cumulative fluorescence intensity analysis, all WNV-POL mutants are active while comparing to Wild-type $(100 \%)$, the RdRp activity is $88.18 \%$ of $69-70,54.32 \%$ of R398A, 39.76\% of AMC and $14.6 \%$ of inactivemutant. The molecular-docking results showed that all the three compounds with high binding energy and good affinity make molecular contacts with the Motif $\mathrm{F}$ of the finger domain.

All results conclude that mutants are active but the activity is lesser as compared to wild-type. Based on that study, the host factors which interact with RdRp for nuclear localization can be used to design small molecule inhibitors against the key-step in viral lifecycle. Molecular-docking analysis revealed that heterocyclic compounds identified in silico may be considered as the starting point for the development of antiviral inhibitors targeting.

\section{Pre-transplant seroprevalence and posttransplant reactivation of opportunistic viral infections in solid organ transplant recipients: a single tertiary care centre experience from North India}

\author{
Gupta $S^{1}$, Gupta $E^{1}$, Mahajan $S^{1}$, Pamecha $V^{2}$, Mathur R.P. ${ }^{3}$
}

Email ID for Correspondence: shefali.g2109@gmail.com ${ }^{1}$ Department of Clinical Virology; ${ }^{2}$ Department of Hepatobiliary Surgery, ${ }^{3}$ Department of Nephrology, Institute of Liver and Biliary Sciences, New Delhi, India

Herpes group of viruses' causes significant morbidity and mortality in solid organ transplant (SOT) recipients. Aim was to determine pretransplant seroprevalence of herpes viruses in liver (LTx) and renal transplant (RTx) recipients and donors and determine post transplant viral reactivation. We undertook this retrospective study for 177 patients $\{140$ (LTx) and 37 (RTx) \} from January' 16-March' 2018. Data regarding pre-transplant donor(D) and recipient(R) serological profiles for CMV IgG, EBV IgG, HSV1\&2 IgG AND VZV IgG and post transplant DNA viral loads were collected. Descriptive analysis was done and frequencies calculated. Pre-transplant serological profiles for the donors and recipients are as follows; (CMV IgG: 160 $(90.4 \%) \mathrm{D}+\mathrm{R}+, \quad$ 11(6.2\%)D-R + , $3(1.7 \%) \quad \mathrm{D}+\mathrm{R}-\quad$ and 3(1.7\%)D-R-)\}, \{EBV IgG: $159(89.9 \%) \mathrm{D}+\mathrm{R}+, 13(7.3 \%) \mathrm{D}-$ 
$\mathrm{R}+, \quad 3(1.7 \%) \mathrm{D}+\mathrm{R}-\quad$ and $\quad 2(1.1 \%) \mathrm{D}-\mathrm{R}-)\}, \quad\{(\mathrm{HSV} \quad \mathrm{IgG}:$ $78(44 \%) \mathrm{D}+\mathrm{R}+, \quad 55(31 \%) \mathrm{D}-\mathrm{R}+, \quad 13(7.3 \%) \mathrm{D}+\mathrm{R}-\quad$ and 31(17.7\%)D-R-) \} and VZV IgG: $120(67.8 \%) \mathrm{D}+\mathrm{R}+$, 32(18\%)D$\mathrm{R}+, 21(11.9 \%) \mathrm{D}+\mathrm{R}-$ and 4 (2.3\%)D-R.Post-transplant CMV DNA was positive in 41(23.1\%); [37(90.25\%) LTx and $4(6.75 \%)$ RTx recipients. Out of $41,7(17.7 \%)$ were high risk $(D+R-g), 28$ $(68.3 \%)$ intermediate risk $(\mathrm{D}+\mathrm{R}+)$ and for remaining 6 patients serostatus could not be determined. Post transplant EBV DNA was positive in $2(4.8 \%)$ LTx patients and both were high risk (D + R-). Median CMV viral load was higher in RTx $\left(3.5 \times 10^{3}\right)$ than LTx $\left(5.0 \times 10^{2}\right)$ copies $/ \mathrm{ml}(p$ value $<0.005)$. CMV disease was seen in 26/41(63.4\%) while 2 LTx patients had tissue invasive disease. Posttransplant CMV reactivation was highest in first month [24 (58\%)], followed by second [10(24.3\%)] and third month [7(17.7\%)]. Median time of occurrence of CMV was 25 days (IQR: 13.5-48 days). Pretransplant serological work-up and documentation of herpetic viral infections (CMV and EBV DNA viremia) as early as in the 1st month of post-operative period might alert clinicians and guide in effective patient management. However, more studies are needed to confirm our findings.

\section{Serum micro RNA-155 and microRNA-21 as a potential non-invasive biomarker to track Japanese Encephalitis virus infection among Acute Encephalitis syndrome patients}

Baluni $M^{1}$, Fatima $T^{1}$, Ghildiyal $S^{1}$, Singh $D^{1}, \operatorname{Kumar} P^{1}$, Zia $A^{1}$, Tiwari $\mathbf{R}^{1}$, Reddy $\mathbf{H}^{2}$, Dhole $\mathbf{T}^{2}$

Email ID for Correspondence: tndhole@sgpgi.ac.in

${ }^{1}$ Sanjay Gandhi Post Graduate Institute of Medical Sciences, Lucknow, Uttar Pradesh, India; ${ }^{2}$ King George Medical University, Lucknow, Uttar Pradesh, India

Japanese encephalitis virus (JEV) is a neurotropic mosquito-borne Flavivirus, mainly prevalent in Asia. It is the most important causative agent of acute viral encephalitis in humans. Recently, microRNAs (miRNAs) are discovered as a key regulator of inflammatory and immune responses in various diseases including neurological and viral infections. Thus, this study was proposed to explore the role of miRNAs in JEV pathogenesis and to check whether changes in cellular miRNA expression due to JE virus infection, can be detected in circulation which would be helpful in diagnosis and treatment. miRNAs (miR-155 and miR-21) were analyzed in the serum of JEV infected patients using quantitative reverse transcription polymerase chain reaction (RT-qPCR). PCR data was normalized using both exogenous (cel-miR-39) and endogenous (hsa-miR-93) controls and relative expression level of genes between groups was calculated using $2^{-\Delta \mathrm{CT}}$ method.

Expression of miR-21 was found significantly upregulated ( $p=0.003)$ in JEV infected patients as compared to healthy controls whereas, miR-155 showed a significant decrease in JE patients when compared to healthy controls $(p=p=0.004)$. A negative correlation was observed between miR-21 $(\mathrm{r}=-0.31)$ and miR-155 $(r=-0.22)$ levels and JE severity. Our finding of changes in serum miR-155 and miR-21 in JE patients reveals that microRNAs deserve investigations as potential biomarkers in disease activity which would be helpful in specific and accurate diagnosis. Correlation study between miRNAs and JE severity suggested that miR-155 and miR21 can serve as new component to develop possible therapeutic strategies for JE in near future.
Infographics vs. text: a survey on symptom based completion rates of test report forms

\section{Ganesan $M^{1}$, Sekar $S^{1}$, Swarna $S^{1}$ and Sudha $M^{1}$}

Email ID for Correspondence: drswarnasenthilkumar@gmail.com VRDL Department of Microbiology ${ }^{1}$, Govt. Theni Medical College, Theni

Successful diagnosis and treatment provided to patients is always associated with symptom based clinical investigations as these symptoms will offer critical clues to the diagnosis. The clinical history part of Test Request Form (TRF) is often incomplete or even sometimes unattended. There is a need to enhance the attention of physician and to allow paramedical personnel to complete the form as physicians are mostly engaged in clinical examination. Hence, an attempt to improve the completeness of clinical history in TRF with Infographics (TRF-I) was evaluated. In this study, equal chance for filling up of both TRF and TRF-I was given to clinicians (in ward) and paramedical persons (at sample receiving point). A total of 87 TRFs were compared for the completion rates of symptoms, of these, percentage of unattended forms were significantly reduced from $23 \%$ (TRF) to $6.2 \%$ (TRF-I). The percentage of filling each of the symptoms was found to be increased and while using TRF-I, greater proportion with more than one symptom per patient was recorded. Symptoms such as cough, chills, throat pain and knee pain was noted as $>80 \%$ which was almost neglected in TRFs. If the relative value of each symptom is considered, combinations of symptoms can provide greater reliability in the diagnosis of viral diseases. In general, visual content attracts the attention and motivates to complete the request form. Thus, the current infographics based filling up of clinical history showed a greater improvement and adopting infographics in request form will be better for future use in diagnostic laboratories, primary care and epidemiological studies.

\section{Role of mitochondrial changes in rabies pathogenesis: a study on post-mortem human brain samples}

\author{
Harsha PK ${ }^{1}$, Mani RS ${ }^{1}$, Srinivas Bharath $\mathbf{M M}^{2}$, Mahadevan $\mathbf{A}^{3}$ \\ ${ }^{1}$ Departments of Neurovirology; ${ }^{2}$ Neurochemistry and \\ ${ }^{3}$ Neuropathology, National Institute of Mental Health \& \\ Neurosciences (NIMHANS), Bangalore
}

India has the highest burden of human rabies accounting for almost a third of the 61,000 global human deaths due to rabies. The major hurdle in the development of effective therapy for rabies is the lack of understanding of the basic mechanisms involved in the pathogenesis of the disease. Recent studies on cell lines using laboratory-adapted rabies virus have indicated the possible role of mitochondrial dysfunction in rabies pathogenesis. This study was therefore undertaken to examine the role of mitochondrial changes in human brain samples obtained post-mortem from patients who succumbed to rabies. Postmortem brain tissues from ten confirmed cases of human rabies, and age-matched non-infected controls each were sourced from the Human Brain Tissue Repository, Department of Neuropathology, NIMHANS. Brain mitochondria were prepared from frontal cortex, hippocampus, cerebellum, and temporal regions of each brain. Mitochondrial respiratory enzymes complex assays (complex I, II, III and IV) were performed using standardized methods. Citrate synthase assay was performed and used as an indicator of mitochondrial mass internal control for complex assays. The activity of respiratory chain complex I activity and complex IV activity were significantly higher in all the four regions of rabies infected brains compared to non- 
infected controls. However, activity of complex II and III did not reveal a significant difference in brain samples from rabies-infected cases Vs controls. Results of this first study on rabies-infected human brain sample suggest a possible role of mitochondrial dysfunction in rabies pathogenesis. The findings of this study may help in developing novel therapeutic approaches for treatment of rabies.

\section{Comparison of influenza A H1N1 Pdm09 activity in central India between 2015 and 2017}

\section{Nema RK ${ }^{1}$, Gupta $S^{1}$, Agarwal $A^{1}$, Sharma $R^{1}$, Kudsia A, Nema S, Biswas D}

Email ID for Correspondence: ramkumar.rvl@aiimsbhopal.edu.in Regional Virology Laboratory, All India Institute of Medical Sciences Bhopal ${ }^{1}$, Saket Nagar, Bhopal, 462020 India

Influenza A H1N1 pdm09 strain accounted for the predominant Influenza activity in our region in 2015 and 2017; while it was relatively dormant in 2016. We surmised that emergence of novel viral strains are associated with increased pathogenicity and transmissibility of the virus and, hence, should reflect in the disease characteristics of the outbreaks occurring in 2015 and 2017. We, therefore, were interested in comparing the seasonality, demography, disease burden and clinical characteristics of these two outbreaks. Nasopharyngeal/oropharyngeal swab samples of Influenza-suspects were referred to our laboratory from 17 district hospitals and 4 tertiary care teaching hospitals of Madhya Pradesh throughout the study duration. These samples were processed using CDC RT-PCR protocol using CFX96 Connect Thermal Cycler (Bio-Rad). In 2015 and 2017, $22.18 \%(431 / 1934)$ and $23.24 \%(639 / 2749)$ of Influenza-suspects were positive for InfA, of whom InfA H1N1 pdm09 strain accounted for $88.86 \%$ (383/431) and $84.19 \%$ (538/639) of cases. In contrast, in 2016 only $10.92 \%(113 / 1034)$ of Influenza-suspects were positive for InfA H1N1 pdm09 strain. Comparing the seasonality of outbreaks, we observed that in 2015 peak Influenza activity was in March while in 2017 the same was recorded in September. Predominant age-group affected in 2015 was 21-30 years (20\%), while in 2017 patients belonging to $0-5$ years and 51-60 years were predominantly affected $(16.44 \%$ and $16.63 \%)$. There was a significantly higher proportion of severely ill patients (category C; guidelines of MoHFW Government of India) in $2017(72.72 \%$ vs. $48.95 \%)(P<0.001)$. The differences in the seasonality, demographic and clinical severity of the outbreaks suggest the likelihood of the emergence of novel viral variants in this region between 2015 and 2017. It would be prudent to verify this possibility through whole genome sequencing of a representative set of samples from the two outbreaks.

\section{Exotic human viruses of India}

\section{Poduri C D}

Email ID for Correspondence: cdpoduri@gmail.com Independent Researcher and Freelance Writer

Infections of a number of exotic human viruses have been reported from India. In the present study, epidemiology of seven virusesChandipura Virus (ChpV), Chikungunya virus (ChikV), CrimeanCongo Haemorrhagic Fever Virus (CCHFV), Ebola Virus (EbV), Middle East Respiratory Syndrome Corona Virus (MERS-CoV), Nipah Virus (NiV), Zika virus (ZikV)—is collated with respect to India. Search engines/databases Pro-Quest Central, JSTOR, PubMed, Google Scholar were used to retrieve appropriate articles reporting confirmed cases of the mentioned seven viruses. Only for ChikV this condition was not applied. Articles were also retrieved with an aim to identify the first confirmed report of the mentioned viruses. Except for MERS-CoV, all the studied viruses have at least one confirmed case in India. Viruses like ChikV, ChpV, CCHFV and NiV displayed multiple epidemics since their first report. Increasing prevalence of the studied viruses evidently suggests that India might be an endemic country with respect to some of these viruses. The reasons could be many. But the presence of right conditions and vectors for these viruses to spread visibly indicates that India can no longer ignore them. In this scenario, regular monitoring for these viruses becomes mandatory.

\section{Identification of potential circulatory microRNA as candidate biomarker for liver cancer in Hepatitis B virus infection}

\section{Neeti Nadda ${ }^{1}$, Sonu Kumar ${ }^{1}$, Shashi Paul ${ }^{1}$, Anoop Saraya ${ }^{1}$, Shalimar $^{1}$ \& Baibaswata Nayak}

Email ID for Correspondence: baibaswat@gmail.com Departments of Gastroenterology and Radio diagnosis, ${ }^{1}$ All India Institute of Medical Sciences, Ansari Nagar, New Delhi, India

Hepatocellular carcinoma (HCC) is the second leading cause of cancer-related mortality worldwide. Major risk factor for $\mathrm{HCC}$ is underlying Hepatitis B virus (HBV) infection as compare to other causes of chronic liver disease. HBV mediated liver cancer occurs due to chronic inflammation, insertional mutagenesis of viral genome or trans-activation of viral protein. The microRNAs (miRNAs) are small non-coding RNA released to circulation and aberrantly expressed many diseases including viral disease and cancer. These miRNAs can be considered as circulatory biomarker due to ease of detection and high stability in circulation. In HBV induced HCC, the potential of these miRNA as early cancer biomarker or marker for cancer progression need to be evaluated. We have screened 84 circulatory miRNAs that are differentially expressed in liver cancer using miRNA PCR Array. The miRNAs relevant to HBV-induced liver cancer were selected by differential expression in serum of healthy control, treatment naïve chronic hepatitis B (CHB) patient, HBV induced cirrhotic and HCC patients $(n=40)$. Total RNA isolated from serum were polyadenylated, reverse transcribed and amplified by real time PCR. The differential expression was analyzed by comparative $\mathrm{Ct}$ method after normalization using exogenous $\mathrm{Cel}$ miR39. Differential expression of 84 miRNAs in serum of $\mathrm{CHB}$ naïve, cirrhotic and HCC patients were analyzed by normalization with healthy control. Twelve miRNAs (miR-126, 17, 195, 19b,19a, 20a, 223, 222, 25, 30d, 92a and miR-16) were differential upregulated ((1.12 to 19 fold) and four miRNAs (miR-100, miR-210, miR-423 and let-7c) were down-regulated ( 0.4 to 1.18 fold) in $\mathrm{HCC}$ as compare to cirrhotic and CHB patients. Both upregulated (miR-25-3p and miR92a-3p) and down regulated (miR-let-7c and miR-100-5p) miRNA were evaluated in validation cohort of healthy, $\mathrm{CHB}$ and $\mathrm{HCC}$ patients. These circulatory miRNAs have potential for cancer marker in $\mathrm{HBV}$ infection. 
Activity of haemorrhagic fever viruses in North East Region of India

\author{
Prasad AK ${ }^{1}$, Phukan $\mathrm{AC}^{2}$, Barman $\mathrm{B}^{3}$ \\ ${ }^{1}$ Department of Microbiology; ${ }^{2}$ Department of Medicine; \\ ${ }^{3}$ North eastern Indira Gandhi Regional Institute of Health and \\ Medical Sciences, Shillong India
}

Viral Haemorrhagic Fever (VHF) is a major public health problem of North East (NE) India. The present study was undertaken with the objective to study the etiology of VHF of Dengue, Chikungunya and Crimean Congo Haemorrhagic Fever (CCHF) virus origin among patients attending tertiary health care centre in NE India and also to study the clinical profile of such patients. Serum was collected from 51 suspected VHF patients and IgM antibody capture ELISA was employed to detect anti-Dengue and anti-Chikungunya IgM antibody. The samples were also tested by real-time RT-PCR for detection of Dengue, Chikungunya and CCHF specific nucleic acid. The demographic, clinical and laboratory profile of the patient was noted in detail. Serum samples of 16 of 51 suspected cases were confirmed to be suffering from VHF. Among the confirmed cases, 12 were diagnosed with Dengue Haemorrhagic Fever, one was diagnosed with Chikungunya and there were three with Dengue and Chikungunya virus co-infection. However, CCHFV infection was not detected in our study. Male preponderance was seen among the infected patients where 20-30 year age group was found to be predominantly affected. Among confirmed cases fever (100\%), headache (93.8\%), petechial rashes $(68.8 \%)$, ascites (37.5\%) and conjunctival suffusion $(18.8 \%)$ were the major clinical findings. Two patients were diagnosed with Dengue Shock Syndrome who later succumbed to the illness. Most patients $(41.2 \%)$ were farmers by occupation from Meghalaya and nearby Arunachal Pradesh. Information generated from this study may be used for further in depth molecular epidemiology with characterization of the circulating strains of the viruses in the NE region of the country. This study further reiterates the fact that CCHF virus infection in humans is still probably absent in this region despite recent serological evidence of its activity in sheep, goat and bovine population of NE India.

\section{Phylodynamics of circulating genotypes in a hospital based rotavirus surveillance study during 2008-2017}

\section{Lo Ma, Banerjee Aa, Mullick Sa, Dutta Sa, Chawla-Sarkar Ma ${ }^{1}$}

Email ID for Correspondence: chawlam70@gmail.com Division of Virology, National Institute of Cholera and Enteric Diseases $^{1}$, Kolkata, India

Group-A human rotaviruses (GARV) are among the leading etiological agents responsible for acute infantile gastroenteritis worldwide. In an on-going hospital based diarrhoeal diseases surveillance in Kolkata, Eastern India (2008-17), GARV was identified as the most common cause of infantile gastroenteritis. The circulating strains were genotyped and characterized to understand their phylodynamics prior to introduction of vaccine in India. Stool samples were screened from children ( $\leq 5$ years) with diarrhea, seeking health care facilities at two hospitals in Kolkata. Screening for VP6 antigen was done by ELISA. GARV positive samples were genotyped by multiplex semi-nested PCR and subsequent nucleotide sequencing of VP7 (G- type) and VP4 (P- type) gene segments. Among 7814 stool samples, 2895 (37.04\%) were found to be GARV positive. Highest proportion of GARV positivity was found in children of 6-12 months age group. The rate of infection peaked during cooler months of November to February. G1, G2, G3, G9 and G12 in conjunction with $\mathrm{P}[4], \mathrm{P}[6]$ and $\mathrm{P}[8]$ genotypes were seen to cocirculate in the population. Incidence of G1 peaked in 2008-10 and 2013-15. Meanwhile, G9 was seen to be the preponderant strain of 2011-2013. A sharp deflection from G1 to G3 strains was observed during 2016-2017. Polymorphic G3 strains were first reported in Eastern India during this study period. All the strains clustered distantly from the vaccine strains in the phylogenetic dendrograms, depicting less genetic relatedness. Differences in amino-acid residues encompassing the antigenic epitopes of VP4 and VP7 capsid proteins between vaccine and Kolkata strains were observed. GARV shows a tendency towards directional selection under persistent immunological pressure that might promote natural selection of one genotype over another in a particular geographical area. Therefore, surveillance of the circulating GARV genotypes shall underscore the importance of introduction of rotavirus vaccine in this region.

\section{Changing pattern of Dengue infection in the state of Odisha, Eastern India}

\section{Subhra Subhadra, Jyotsnamayee Sabat, Bhagirathi Dwibedi,} Subrata K Palo Sanghamitra Pati

VRDL, ICMR- Regional Medical Research Centre, Bhubaneswar, Odisha

Dengue has emerged as a major public health challenge in terms of both clinical pattern and epidemiology. Odisha reported first dengue epidemic in year 2010 and continued to report cases each year in epidemic form during post monsoon period becoming an endemic phenomenon in the state. Present study depicts the changing pattern of Dengue infection with reference to its serotypes and genotypes. The study included 5320 suspected Dengue cases referred from different health facilities of the state during 2010-2017. Dengue NS1 antigen and IgM antibody was done through ELISA. Serotyping was done for the samples positive for NS1 antigen through RT PCR by amplifying a part of core-pre- Membrane gene (CprM) followed by sequencing and phylogenetic analysis. Dengue NS1 antigen was detected in $53.2 \%$ of cases and IgM antibody in $17.7 \%$ cases. Dengue serotype 2 (DEN-2) was the only serotype detected in 2010 and 2011 whereas all four serotypes, 1,2,3,4 (DEN-1, DEN-3, DEN-4) were detected among cases during 2012-2017. Mixed serotypes were detected in $15 \%$ of cases; DEN-2 being dominant (76\%). Phylogenetic analysis revealed genotype IV of DEN-2 and genotype III of DEN-1 and 3 are mainly circulating in this region. High prevalence of Dengue was observed with increased trend from 2010 to 2017 affecting both urban and rural areas. Severity of illness was found to be associated with mixed infection, however in majority of the cases, it was mild to moderate indicating the endemic nature of disease in most parts of the state.

\section{Adjuvant role of a NKT cell activating glycolipid with anti-rabies vaccine}

\section{Nagaraju R, Venkataswamy MM}

Email ID for Correspondence: ruthunagraj@gmail.com Department of Neurovirology, NIMHANS, Bengaluru

Rabies, a zoonotic disease caused by rabies virus, is a major public health concern in many parts of the world, particularly Asia. Worldwide, more than 55,000 human deaths occur per year due to 
rabies, of which 20,000 are from India. Stray dog populations serve as principal reservoirs and transmitters for rabies virus in India. The current pre-exposure anti-rabies vaccination for stray dogs involves two priming doses followed by annual boosters, which is a daunting task. Newer simple vaccination approaches are essential for improved control of rabies. In this proof of concept study, we investigated the role of Natural Killer T (NKT) cell based glycolipid adjuvant in enhancing anti-rabies vaccine immunogenicity in the murine model. C57BL/6 mice $(n=4)$, were immunized subcutaneously with either 3 doses of rabies vaccine alone or one dose of rabies vaccine with glycolipid adjuvant. After 3 weeks, serum samples were tested for Neutralizing antibody titers $(\mathrm{Nab})$ by the rapid fluorescent focus inhibition test (RFFIT). Antigen specific T- cells producing cytokines, IL-4 or IFN- $\gamma$, were estimated by ELISPOT assay. Nab titers in mice immunized with a single dose of vaccine with adjuvant were higher (twofold) than mice with 3 doses of vaccine alone (1.11 IU against $0.53 \mathrm{IU})$. The frequency of $\mathrm{T}$ cells secreting cytokines were higher in mice receiving combined vaccine and adjuvant, IL-4 (two-threefolds) and IFN- $\gamma$ (one-twofolds), as compared to mice receiving vaccine alone. This study suggests that the glycolipid adjuvant can potentially enhance the rabies vaccine specific antibody titers as well as Th1 and Th2 cellular immune responses in the murine model.

\section{Prevalence of hepatitis $B$ and $C$ virus in pregnant females in a tertiary care hospital}

\section{Tuli A, Singla N, Gill M, Chander J}

Email ID for Correspondence: arpantuli@googlemail.com Department of Microbiology GMCH-32 Chandigarh

Viral hepatitis in pregnant females through vertical transmission contributes to the compromised prognosis in the new-born along with increased risk of maternal complications. Perinatal transmission of hepatitis B occurs if the mother has had acute infection during late pregnancy, or if she is a chronic HBsAg carrier while hepatitis $\mathrm{C}$ transmission occurs mostly around time of delivery. This information about prevalence of viral hepatitis during pregnancy is essential for the health and programme planners. Thus, the current study aimed at investigating their prevalence amongst antenatal women attending the tertiary care hospital. This study was a hospital based cross-sectional study that included 3070 pregnant women who attended the antenatal clinic of the tertiary care hospital, during the periods of Jan 2017June 2018. The sera collected from the patients were tested for $\mathrm{HBsAg}$ and anti-HCV using ELISA kit. Positive and negative control serum samples were run alongside test. Out of total 3070 pregnant females, 100 were detected positive for HBsAg which accounts for $3.3 \%$, and 40 were detected positive for HCV which accounted for $1.3 \%$. Most common age group with $\mathrm{HBV}$ and $\mathrm{HCV}$ infection was 20-29 years. Most of the positive pregnant females belonged to rural background. Universal free screening of all pregnant women for $\mathrm{HBV}$ and $\mathrm{HCV}$ should be done to prevent the next generation from being grappled by chronic hepatitis, cirrhosis and hepatocellular carcinoma. Screening will reduce the prevalence, risks of infection and its transmission to the new-born.

\section{Congenital CMV infection: a report from a tertiary care center in Chandigarh}

Singla $\mathbf{N}^{1}$, Jain $\mathrm{S}^{\mathbf{2}}$,Kaur $\mathbf{P}^{\mathbf{1}}$, Chander $\mathbf{J}^{1}$

Email ID for Correspondence: nidhisingla76@gmail.com Department of Microbiology ${ }^{1}$, Neonatology ${ }^{2}$ Government Medical College Hospital, Chandigarh

Human cytomegalovirus is increasingly being recognized as important causes of congenital infection. The incidence of congenital CMV ranges from 0.5 to $3.0 \%$ in all live births. Intrauterine transmission of CMV to the child can occur irrespective of prior maternal exposure. Most of the congenitally infected infants (85-90\%) are asymptomatic at birth but $5-15 \%$ of them develop sequelae mostly as sensorineural hearing loss, visual impairment or delay of psychomotor development. Dense population, overcrowding, low literacy, poor sanitation, unhygienic conditions, etc., are among the various reasons predisposing people of developing countries and even poorer society to be exposed to CMV. In children suspected of congenital CMV infection, the seroprevalence of CMV IgM has been found to be $12.5-20 \%$ by various authors. The blood samples were received from the infants especially those with severe intrauterine growth retardation or for small for age neonates for determining the $\operatorname{IgG}$ and $\operatorname{IgM}$ antibodies against CMV infection over the last 1 year and 6 months (January 2017 to June 2018). The tests were put up by $\mu$-capture ELISA for CMV IgM and IgG antibodies as per the manufacturer's instructions for qualitative detection. Interpretation of the results was done on basis of controls provided with the kits. A sample was said to be positive for IgM or IgG antibodies if the absorbance value was more than cut-off value. Eleven infants were positive for CMV IgM antibodies during the time period. They were suspected with having congenital CMV infection. The case histories of these infants were studied retrospectively and the details were noted. The age of the children varied from as less as 5 days to 1 year. Congenital CMV is more prevalent than we think and the clinicians should be on lookout for the disease as early identification of the congenital or perinatal CMV infection.

\section{Association of interleukin-1/ (-511 C/T) and interleukin-10 (-1082 $\mathrm{A} / \mathrm{G})$ gene polymorphism with Japanese encephalitis disease in North Indian population}

Ghildiyal S, Baluni M, Fatima T, Singh D, Zia A Gaur P, Tiwari R, Dhole T N

Email ID for Correspondence: tndhole@sgpgi.ac.in Sanjay Gandhi Post Graduate Institute of Medical Sciences, Lucknow,UP, India

Japanese encephalitis (JE) is the most common form of viral encephalitis in many parts of Asia. It is caused by Japanese encephalitis virus which belongs to the family Flaviviridae. JE is associated with neuroinflammation in central nervous system and disruption of Blood brain barrier (BBB). Pro-inflammatory cytokines and anti-inflammatory cytokines play a vital role in JE as well as other neurotropic viral diseases. This study evaluated the association of single nucleotide polymorphisms of interleukin (IL)-1 $\beta(-511)$ and IL$10(-1082 \mathrm{~A} / \mathrm{G})$ with JE virus susceptibility in northern Indian population. We performed a case-control study using 85 patients and 85 healthy controls. The polymorphism study has been performed by restriction fragment length polymorphism (RFLP). We analyzed the 
$\mathrm{C} / \mathrm{T}$ and $\mathrm{C} / \mathrm{C}$ genotypes of IL- $1 \beta$ were significantly associated with higher risk of JE infection ( $p=0.000$ and $p=0.007$ respectively) whereas A/G genotype of IL-10 was associated with reduced risk of JEV infection $(p=0.001)$. Among severe cases of JE, only CT genotype of IL-1 $\beta$ was has been found to be associated with disease severity $(p=0.02)$. IL-1 $\beta(-511 \mathrm{C} / \mathrm{T})$ may affect the host susceptibility to Japanese encephalitis in North Indian population. IL-1 $\beta$ (-511C/T) and IL-10 (1082 A/G) polymorphism may be associated with severity of Japanese encephalitis disease.

\section{Association of TLR8 gene polymorphisms in two different groups with the risk of Cytomegalovirus infection among pregnant women in North Indian population}

\author{
Tiwari $R^{1,2}$, Fatima $T^{1}$, Baluni $M^{1}$, Ghildiyal $S^{1,2}$, \\ Srivastava J.K ${ }^{2}$, Dhole T.N. ${ }^{1}$ \\ Email ID for Correspondence: tndhole@gmail.com \\ ${ }^{1}$ Sanjay Gandhi Post Graduate Institute of Medical Sciences, \\ Lucknow, ${ }^{2}$ Amity University, Lucknow
}

Human cytomegalovirus (HCMV) causes the most common intrauterine infections, affecting approximately 40-100\% of pregnant women. Taking into account the immune response to HCMV, the Toll-like receptors (TLRs) have been reported to play important role. Data from various studies suggested that the ability of certain individuals to respond properly to TLR ligands may be impaired by single nucleotide polymorphisms (SNPs) within TLR genes, resulting in an altered susceptibility to, or course of, infectious or inflammatory disease. In TLR8, the common Met1Val missense polymorphism has been implicated in a number of diseases such as tuberculosis and progression of HIV infection. However, data on the relation between HCMV infections and TLR8 polymorphisms are absent in the literature. Therefore, the current study was aimed to describe the role of TLR-8 (Met1Val and $129 \mathrm{C} / \mathrm{G}$ ) gene SNPs in the occurrence of HCMV infection among pregnant women and healthy control subjects in Northern India. In this case-control study, blood samples were collected from 400 pregnant women, including 150 women infected with HCMV, and 250 control women without HCMV infection. Infection in pregnant women was determined by HCMV DNA detection. Further TLR8 allele-specific polymorphism using PCRRFLP was performed and result was analysed using $\chi^{2}$ test. In the current study, G/G genotypes at TLR-8 Met1Val genotype was significantly associated with risk for HCMV infection $(p=0.003)$ whereas, TLR-8 -129 C/G genotype did not show any such association. Similarly, the frequency of TLR- 8 Met $1 \mathrm{Val} G$ allele was higher in case group as compared to control group and is associated with risk of disease progression $(p=0.013)$. The current study demonstrates that G/G genotypes of TLR 8 met1 val increase the risk of development of HCMV infection therefore we can say that possibly predispose pregnant women to $\mathrm{HCMV}$ infection, thus increasing the risk of congenital cytomegaly development.
Evaluation of antiviral efficacy of halogen-substituted flavonoids in Chikungunya virus

\author{
Rani $\mathbf{R}^{1}$, PuranikNinad $V^{2}$, Singh $V^{1}$, Srivastava $P^{2}$, Tomar $S^{1}$ \\ Email ID for Correspondence: ruchirani022@gmail.com \\ ${ }^{1}$ Department of Biotechnology, Indian Institute of Technology \\ Roorkee, Uttarakhand,India; ${ }^{2}$ Bioprospecting Group, \\ Agharkar Research Institute, Pune, Maharashtra
}

Chikungunya virus (CHIKV) is a positive, single-stranded RNA virus of $\sim 11.8 \mathrm{~kb}$ long.It belongs to the family of Togaviridae and genus Alphavirus that is typically transmitted by mosquitoes. It has three genotypes depend upon the geographical distribution-Asia, West African and East Central South African. CHIKV contains 2 ORFs, one on the $3^{\prime}$ end and other on the $5^{\prime}$ end. The $3^{\prime}$ end ORF producing the structural proteins while the $5^{\prime}$ end producing four non-structural proteins (nsP1-4).After 2016, CHIKV causes the major outbreak in India.

Flavonoids possess various biological and pharmacological activities that are being exploited to develop antiviral and antibacterial drug molecules.In this study, Flavonoid based inhibitors against infectivity and replication of CHIKV has been investigated.Three flavonoid compounds (Compounds- $\mathrm{ABr}, \mathrm{BCl}$, and $\mathrm{CF}$ ) that are halogenated at various positions were used and the inhibition potential of these compounds against clinical strain of CHIKV has been investigated. The relative antiviral activities of these flavonoids compounds against CHIKV were determined by observing the Cytopathic Effect(CPE) followed by the plaque reduction assay and then by quantitative real-time PCR.These assay proved to be reliable and rapid for determining inhibitory concentration as well as inhibitory effect well with clinically achievable drug levels. Compound $\mathrm{ABr}$ shows 92, 86 and $45 \%$ inhibition in the $70 \mu \mathrm{M}, 50 \mu \mathrm{M}$, and $30 \mu \mathrm{M}$ concentration range respectively while in the same concentration range compound $\mathrm{BCl}$ shows $95 \%, 88 \%$ and $56 \%$ inhibition respectively but Compound $\mathrm{CF}$ had no antiviral activity in these test system. Very similar results were deduced from the CPE images. So, as the concentration of inhibitor increase the inhibitory effect on $\mathrm{CHIKV}$ increases as compared to control where there is no inhibitor.Thus, the compound $\mathrm{ABr}$ and $\mathrm{BCl}$ may be considered as a good inhibitor target against CHIKV.Further biochemical and structural based investigations to identify the molecular target of these compounds is in progress.

\section{Modulation of Herpes Simplex Virus-1 infection by MBZM-N-IBT}

\author{
Gangamma $S^{1}$, Jheelam Sarkar ${ }^{1}$, Veeksheetha ${ }^{1}$ \\ and Krishnaja Saseendran ${ }^{1}$
}

Email ID for Correspondence: ashirbadsaikatde@gmail.com National Institute of Technology Karnataka Surathkal ${ }^{1}$, Mangaluru, India

Life-long latency is usually established for HSV-1 infection and that leads to recurrence of the infections. It is known to come central nervous system infection leading to serious illness, in the immunocompromised population. It is also known to cause central nervous system infection leading to serious consequences. Emergence of drug resistance to Herpes simplex viruses (HSV) and cross resistance across nucleoside inhibitors has warranted development of new nonnucleoside antivirals. MBZM-N-IBT (1-[(2-Methyl benzimidazole-1yl) Methyl]-2-Oxo-Indolin-3-ylidene] Amino] Thiourea (MBZM-NIBT)), was investigated in this study for its capacity to inhibit HSV- 
1.As molecular docking analysis suggested its inhibitory capacity against HSV-1. In order to understand the effect of MBZM-N-IBT in HSV-1 infection, plaque assay and confocal microscopy were carried out. Viral RNA and protein levels were assessed by RT-PCR Western blot and FACS analysis.Time of addition experiment was performed to understand the possible stage of HSV-1 infection that is affected by MBZM-N-IBT. We have shown that the HSV-1 infectious viral particle formation was abrogated by $99 \%$ in presence of MBZM-NIBT. Viral mRNA was also reduced by $72 \%$ and $84 \%$ for UL 9 and gC respectively. MBZM-N-IBT also reduced the protein synthesis for $\mathrm{gC}$ and ICP8 significantly. While mRNA of ICP8 was not significantly affected, its protein synthesis was reduced by $47 \%$. Further, time of addition experiment revealed the capacity of MBZM-N-IBT to inhibit HSV-1 at early, middle and late stages of infection. Other than inhibiting ICP8, this was attributed to the probable inhibition of multiple targets including ICP27, UL42, UL25, UL15 and gB which are involved in various stages of HSV-1 infection. This is a significant observation considering the fact that unlike nucleoside inhibitors (acyclovir), MBZM-N-IBT is found to target the multifunctional viral protein (ICP8). Hence, this can further be validated and optimized for the development of a non-nucleoside inhibitor as an alternative HSVantiviral.

\section{Abrupt change in distribution of Dengue virus serotypes over a period of two consecutive years in central India}

\author{
Yadav AK ${ }^{1}$, Agarwal A ${ }^{1}$, Ansari $K^{1}$, Prakash $A^{2}$, Biswas $D^{1}$ \\ Regional Virology Laboratory, All India Institute of Medical \\ Sciences, Bhopal ${ }^{1}$, Department of Microbiology, Barkatullah \\ University Bhopal, M.P ${ }^{2}$, India
}

The serotype distribution of Dengue demonstrates significant geographical and temporal variation across the globe. The frequency with which this distribution changes in a particular geographical area is not exactly known and data from central India on the serotype distribution of Dengue and its association with different clinical correlates has not been widely reported so far. This study was conducted to compare the relative distribution of Dengue virus serotypes between 2016 and 2017 among cases of Dengue reporting to a tertiary care teaching hospital in Central India. Clinically suspected Dengue cases were confirmed serologically through the performance of NS1 antigen and/ or Dengue IgM capture ELISA. Dengue-confirmed cases were subjected to RT-PCR using genus-specific primers and thereafter serotyped using serotype-specific primers. In 2016 out of 201 Dengue cases, 73 patients were positive for RT-PCR and the circulating serotype found were predominantly DENV-1 (39.6\%) followed by DENV-2 (34\%), DENV-3 (22\%) \& DENV-4 (4.4\%). In the subsequent year of 2017, the distribution of Dengue serotypes abruptly changed from the previous year and predominantly comprised of DENV-3 (43.2\%) followed by DENV-4 (32.8\%), DENV-1 (15.1\%) \& DENV-2 (8.9\%). Mixed infection with multiple serotypes was observed in $21.9 \%(16 / 73)$ and $19.2 \%(42 / 219)$ of cases in 2016 and 2017 respectively. Comparing the duration of illness with the infecting serotype, we observed relatively prolonged viremia beyond 5 days of illness in significantly higher proportion of DENV-2 infected patients $(p=0.001)$. The present study reports a sudden change in the distribution of DENV serotypes over two subsequent years in Central India, which could possibly be the reason for the outbreak in 2017 being almost 3 times larger. We also observed significantly prolonged viremia in DENV-2 infected patients compared to patients infected with other serotypes.
Air pollution and health: modulation of antiviral gene expression and viral entry processes

\section{Gangamma S, Jheelam Sarkar, Veeksheetha and Krishnaja Saseendran}

National Institute of Technology Karnataka Surathkal, Mangaluru', India

According to a World health organization (WHO, 2016) report, acute lower respiratory infections are associated with ambient air pollution exposure and cause significant mortality among children $<5$ years of age. Lower respiratory tract infection is one of the leading causes of death among children in India (Bassani et al. 2010). Viral infection was identified in $49 \%$ of pneumonia cases in children (Ruuskanen et al. 2011). Increased rates of viral infection have been associated with both gaseous and airborne particulate (Cienciwicki and Jaspers 2007). However, much less is known about the underlying mechanisms that link air pollution exposure and increase in susceptibility to respiratory infections (Gangamma 2012). Moreover, there is a relative scarcity of studies on air pollution associated respiratory infections in India (Gangamma 2018). Airborne particulate matter (PM-10) were collected from Indian cities. PM extracts were used for stimulation of A549 and monocytes. After stimulation, viral related host gene or antiviral gene expression was assessed using real time PCR. There is significant evidence that viral infections can stimulate secondary infection by colonized bacteria. Therefore, we also examined the effect of PM and a viral mimic (R848) exposure on phagocytosis. PM treatment up regulated expression of several key host genes such as vATPC, vATPD, TMPRSS2, Furin and Annexin V. Endosome acidification genes such as vATPCandvATPD are essential for fusion and viral entry processes. Taken together our results indicate that PM exposure may enhance viral particle entry into lung epithelial cells. But further experimental confirmation is necessary to understand the mechanisms and biological pathways. Further, our experiments have shown that, R848 exposure significantly reduces phagocytosis. Our preliminary experiments have shown that air pollution exposure may modulate host genes involved in viral entry and innate immunity pathways. Implication of such modulations due to air pollution exposure should be further investigated.

\section{Can rapid dengue diagnostic kits be trusted? A comparative study of commercially available rapid kits}

\section{Atul Garg, ${ }^{1}$ T N Dhole}

Email ID for Correspondence: atulgargsgpgi@gmail.com Department of Microbiology ${ }^{1}$ Sanjay Gandhi Post Graduate Institute of Medical Sciences, Lucknow

Dengue virus infection is an important emerging disease of the tropical and sub-tropical regions and is mainly diagnosed by serological detection of NS1 antigen and IgM anti dengue antibodies. Since ELISA facilities are not easily available at most diagnostic centres, so most of them use various commercially available Rapid Diagnostic Tests (RDTs) kits. This study was designed to access the diagnostic accuracy of 4 commercially available and widely used RDTs for serodiagnosis of dengue virus infection in Indian laboratories. The study was conducted at Department of Microbiology, SGPGIMS, Lucknow and GSVM Medical college, Kanpur to estimate the sensitivity and specificity of following RDTs: (1) Dengue cassette (Panbio, Australia), (2) Bioline Dengue Duo (S D Diagnostics, Korea), (3) Dengue day 1 test (J Mitra and Co, India), (4) 
Dengucheck duo (Tulip Diagnostics, India) on 72 confirmed dengue serum samples that were positive by dengue RT-PCR, dengue NS1 \& IgM ELISA along with 80 serum samples from nondengue febrile illness patients. The majority of the RDTs demonstrated good sensitivity and specificity for detecting NS1 antigen. Detection of antidengue IgM antibodies showed low sensitivity ranging from 27.8 to $77.7 \%$. However specificity was generally moderate (50-100\%) and more consistent across the assays. We conclude that NS1 antigen detection by RDTs is reliable for diagnosis of early acute dengue fever; however RDTs are unreliable to detect IgM antibodies for diagnosing late acute dengue fever and ELISA should be performed as far as possible for serodiagnosis of acute dengue virus infection.

\section{Comparative evaluation of in-house developed and commercial PCR kit for diagnosis of human polyomaviruses (BKPyV, JCPyV)}

\section{Seetha Dayakar, Heera R. Pillai, Radhakrishnan R. Nair}

Email ID for Correspondence: dayakar@rgcb.res.in Laboratory Medicine and Molecular Diagnostics, Srinivasa Ramanujan Institute for Basic Sciences ${ }^{1}$, Rajiv Gandhi Center for Biotechnology,Bio-Innovation Center (BIC), KINFRA Film \& Video Park, Thiruvananthapuram, Kerala, India

The human polyomaviruses BK (BKPyV) and JC (JCPyV) are nonenveloped circular double-stranded DNA viruses, members of the Polyomaviridae family. BKPyV reactivation is mainly related to a renal nephropathy and JCPyV reactivation can induce the progressive multifocal leukoencephalopathy. The aim of this study was to investigate and to compare a novel, rapid multiplex nested PCR and Real-time PCR using samples from patients with suspected nephropathy requiring a renal transplant or immunocompromised patients. Specimens were tested for human polyomaviruses and the results were compared with the commercial assay. The newly developed PCR was used to screen EDTA blood samples from 87 patients attending Government Medical College, Thiruvananthapuram. The method was compared with a commercially available kit employing real-time PCR, for its sensitivity and specificity. Similar pathogen profiles were obtained using both assays for samples positive for any or both viruses. $14(16 \%)$ samples were positive for BK PyV, 7(8.0\%) samples positive for JC PyV and 3 (3.5\%) sample showed co-infection. Based on Cohen's kappa test, the strength of substantial agreement (0.85) of commercial assay was good compared to in-house assay. Overall, our results show that both the Commercial and in-house assays performed similarly in clinical validation. Commercial assays are far more expensive in terms of costs associated with reagents and consumables. The in-house developed PCR assay is fast and reliable and provides results with high specificity and sensitivity. Benefits of use of this in-house assay outweigh the costs of the perhaps more accepted commercial assay.

\section{Comparison of HiMedia's Insta Q series with applied biosystems step one plus real-time PCR machine}

\section{Gohil D}

Email ID for Correspondence: devanshi.gohil@gmail.com Consultant, Research and Development Virology and Molecular Biology ${ }^{1}$ HiMedia Laboratories Pvt. Ltd. Ghatkopar (West), L.B.S. Marg, Mumbai, Maharashtra, India
Real-time polymerase chain reaction (PCR) is considered as a gold standard method for characterization of influenza. HiMedia Laboratories has developed Insta Q series of real-time PCR detection system (Insta Q48 M Series \&Insta Q96). The present study aimed to compare Insta Q series with Applied Biosystems Step One Plus Real-Time PCR system for influenza virus diagnosis. Method: A total of 15 cell culture supernatant samples of influenza A (H1N1) pdm 09, influenza $A$, influenza B viruses and 05 confirmed negative cases of influenza A (H1N1) pdm 09 infections were included in this study. Fifteen (15) healthy volunteer samples were collected and used as controls. Viral RNA extraction and one-step real-time PCR was performed in triplicates using reagents approved by Centre for Disease Control (CDC) for diagnosis of influenza A (H1N1) pdm 09 virus across all nodal centres in India. Results: The Insta Q series suitably diagnosed and typed influenza A (H1N1) pdm 09 virus, influenza A and influenza B virus. The Insta $\mathrm{Q}$ series also had a very good sensitivity with a difference of up to 3 cycle threshold $(\mathrm{Ct})$ values earlier as compared to Applied Biosystems for the detection and quantification of influenza viruses. Conclusion: This preliminary study demonstrates the usefulness of Insta Q series for diagnosis of influenza infections. Greater detection sensitivity of influenza virus using Insta $Q$ series can be attributed to superior bottom-based detection, scanning of individual wells using the flexible robotic arm, LED based excitation source with advanced fibre optics transmission technology and reliable photoelectric detection technology. The Insta Q series is a Truly Open Real-time Platform i.e. compatible with competitor's kits and reagents. Overall, the Insta $\mathrm{Q}$ machines can be used to monitor the dynamics of influenza infections during routine testing and for outbreak preparedness of influenza virus in India and globally.

\section{Etiologic Involvement of Enterovirus and Human Bocavirus in Acute Flaccid Paralysis Cases in India}

\section{Sahu C, Singh D V, Baluni M, Ghildiyal S, Dhole T N}

Email ID for Correspondence: sahu.chinmoy@gmail.com Department of Microbiology, Sanjay Gandhi Post Graduate Institute of Medical Sciences, Lucknow, UP, India 226014

Acute flaccid paralysis (AFP), characterized by the rapid onset of asymmetric paralysis, can be caused by a variety of viral infections or coinfections. Besides wild-type and revertant vaccine strains of polioviruses, several nonpolio enteroviruses (NPEV), Echovirus ECV), Human Bocavirus ( $\mathrm{HBoV}$ ) and Cardiovirus (CV) have also been associated with AFP. Total 586 stool specimens were collected in one year from children suspected for AFP. Molecular method for targeting untranslated region (UTR) and VP1 capsid region was used for detection of human enteroviruses (HEV), human boca viruses (HBoV) and saffold viruses in direct clinical specimen. HEV RNA was detected in 103 (17.6\%) of 586 stool specimens by real time RTPCR targeting the highly conserved UTR region. Out of them, 71 $(12.11 \%)$ were NPEV, partially sequenced by VP1 which revealed the prevalence of echovirus $(\mathrm{ECV}) 19(\mathrm{n}=6), \mathrm{ECV} 11(\mathrm{n}=7), \mathrm{ECV} 18$ $(\mathrm{n}=4)$, ECV $33(\mathrm{n}=5)$, Cardiovirus $(\mathrm{CV})$ A10 $(\mathrm{n}=2)$, CV A9 $(n=1), \operatorname{EV} 80(n=1), \operatorname{EV} 83(n=1), \operatorname{EV} 97(n=2)$ in the pathogens. Total $63(10.75 \%)$ HBoVs were detected by real time PCR which were further sequenced by VP1. Out of them 9 (1.5\%) were detected as co infection with NPEVs. Phylogenetic analysis showed 0.9-5.6\% divergence at nucleotide level among HBoVs. ECV and $\mathrm{HBoV}$ were found as the main etiologic agent in children suspected with AFP. Molecular typing of these viruses is useful for characterizing emerging serotypes and their epidemiological investigation. 
To study the pattern of interleukin-21 production during the clinical course of primary and secondary dengue virus infection

\section{Malik N, Devi B, Oberoi L}

Email ID for Correspondence: diayana23@gmail.com Department of Microbiology, GMC, Amritsar

Dengue is the most important arthropod borne viral infection and due to lack of any commercialized vaccines or accepted pharmacological treatments for DENV disease, patient management becomes the real challenge. Several studies have revealed the clinical relevance of proinflammatory cytokine production during DENV infection, the present study was conducted to evaluate the production of Interleukin 21 , a key soluble mediator mainly produced by CD4 + T cells, during the clinical course of primary and secondary DENV infections and its potential association with disease pathogenesis. The study was carried out in the Department of Microbiology of Government Medical College, Amritsar. Clinically suspected patients of dengue fever presenting to the emergency, outpatient and indoor the Guru Nanak Dev Hospital were included in our study from 1st January 2017 to 30th June, 2018. 100 Samples testing positive for NS1 antigen, designated as confirmed dengue fever cases were included in this study. To differentiate among primary and secondary infections, commercial ELISA kits were used for detecting of titres of IgM and IgG antibodies in patients. All the primary and secondary dengue fever cases were then subjected to quatitative IL-21 analysis by Human IL-21 ELISA Kit. Approval of Institutional Ethics Committee was taken. Confirmed 100 dengue cases were subjected to serological profiling and were differentiated as primary $(50 \%)$ and secondary $(50 \%)$ dengue cases. On the basis of symptomatic profiling as per WHO guidelines, $59 \%$ classical Dengue fever cases, $29 \%$ were DHF and $12 \%$ were DSS. Mean IL-21 levels were significantly raised in the diseased group which was $716 \pm 544 \mathrm{pg} / \mathrm{ml}$ as compared to the healthy controls $75.18 \pm 32 \mathrm{pg} / \mathrm{ml}$. Mean IL-21 levels were more in the secondary dengue cases $(939 \pm 561 \mathrm{pg} / \mathrm{ml})$ as against the primary $(492 \pm 425 \mathrm{pg} / \mathrm{ml})$. It was logical to conduct this study, since an insight into the understanding of disease pathogenesis helps combat the disease in a profound manner. The vision regarding choosing this research was to look for better treatment module for the disease using Interleukin 21.

\section{Isolation of a novel bacteriophage lytic against $E$. faecalis strains}

\section{Bhardwaj S, Mehta M, Sood S, Sharma J., Singh H}

Email ID for Correspondence: sbbhardwaj2002@yahoo.com Institute of Dental Sciences and Hospital, Panjab University, Chandigarh

Enterococci particularly E. faecalis is now recognised as one of the major causes of nosocomial infection. Bacteriophages have effective bactericidal activity and have various advantages over antimicrobial agents. The objective of the study was to isolate a novel bacteriophage against E. faecalis isolates from periodontitis patients.

Gingival crevicular fluid sample was obtained by paper point from periodontitis patients. 46 isolates were identified as Enterococci [39 were E. faecalis $(84.78 \%)$ and 7 were E. faecium (15.21\%)]. Bacteriophage against $E$. faecalis was isolated from sewage sample. The phage was propagated and identified using transmission electron microscope (TEM). TEM microscopy showed that the phage had a hexagonal head $(73 \mathrm{~nm})$ and a $100 \mathrm{~nm}$ long tail resembling phages belonging to Siphoviridae family. The isolated phage was capable of infecting a spectrum of Enterococcal clinical isolates. The role of bacteriophages as strong biotechnological and natural therapeutic agents for Enterococcus faecalis in chronic periodontitis can be considered. Key- Words Bacteriophage, Enterococci, E.faecalis,

\section{Susceptibility towards NPC requires EBV infection and specific host factors}

Nabanita Roy Chattopadhyay, Koustav Chatterjee, Piyanki Das, and Tathagata Choudhuri

Email ID for Correspondence: mailnabanita@gmail.com ${ }^{1}$ Department of Biotechnology, Siksha Vhabana, Visva Bharati, Santiniketan, Bolpur, India

Nasopharyngeal carcinoma (NPC) is a rare, confusing, and commonly misdiagnosed variety of head and neck cancers. Known risk factors for this disease include host genetic factors, Epstein-Barr virus (EBV) infection, and environmental and dietary factors. Though rare worldwide, NPC shows a strong ethnic and geographic bias for its significantly higher occurrence in mongoloid populations including those of North-East India. Therefore, a possible major role of specific host genetic factors is indicated for the generation and promotion of this disease. EBV infection might accelerate the process by its cancer causing properties; whereas other external factors like diet and environmental inhalants, add to the neoplasticity.

\section{Seroprevalence of viral hepatitis in men who have sex with men from Pune, India}

\author{
Mane $\mathbf{A}^{\mathbf{1}}$, Patil $\mathbf{L}^{\mathbf{1}}$, Panchal $\mathbf{N}^{\mathbf{1}}$, Yelgate $\mathbf{R}^{\mathbf{1}}$, Nair $\mathbf{V}^{\mathbf{2}}$, \\ Gangakhedkar $\mathbf{R}^{3}$
}

Email ID for Correspondence: linata.pati1195@gmail.com ${ }^{1}$ ICMR-National AIDS Research Institute, Pune; ${ }^{2}$ Udaan Trust, BudhwarPeth, Pune; ${ }^{3}$ Indian Council of Medical Research, Ansari Nagar, New Delhi, India

Men who have sex with men (MSM) have higher chance of acquiring viral hepatitis. Furthermore a high prevalence of HIV infection among Indian MSM is reported. HIV coinfected individuals, exhibit more rapid progression to liver cirrhosis and hepatic failure. The present study was undertaken to determine the seroprevalence and risk factors associated with acute viral hepatitis among MSM from Pune, India. During 2017-2018, a total of 158 MSM, aged 18-56 years, who selfreported having had oral or anal sex with another man in last 12 months were enrolled in collaboration with Udaan Trust nongovernmental organization, Pune. Blood samples were collected for laboratory detection of HBV surface antigen (HBsAg), anti-HBs antibody, anti-HBc antibody (anti-HBc), anti-HCV antibody, antiHAV IgG and IgM, anti-HEV IgG and anti-HDV IgG. Laboratory results were available for 156/158 samples including, 51 HIV seropositive MSM and 105 HIV seronegative MSM. The mean age for HIV-positive MSM was $29 \pm 8$ years, while for HIV-negative MSM was $29 \pm 8.3$ years, with no significant difference $(p=0.341)$. For HIV seropositive MSM, the mean CD4 count was $560.8 \pm 349.7$ cells $/ \mu \mathrm{L}$. Seroprevalence rates of antiHAV, HBsAg, and anti-HCV in HIV seropositive MSM were $96.1 \%, 5.9 \%$, and $0 \%$, respectively. All $\mathrm{HBs} A g$ positives were also positive for anti-HBc indicating chronic 
Hepatitis B infection. The seroprevalence rates of anti-HAV in HIV seronegative MSM was $95.6 \%$, while all were negative for $\mathrm{HBsAg}$ and anti-HCV. An increasing seropositivity for anti-HAV with advancing age was noted. The prevalence of syphilis was $27.4 \%$ in HIV seropositive and $10.5 \%$ in HIV seronegative MSM. Results show that Hepatitis A is endemic among MSM, however there is a low prevalence of Hepatitis B and C infection indicating that prophylactic hepatitis $\mathrm{B}$ vaccination and safe-sex counseling may help prevent transmission of hepatitis viruses among MSM who may be engaged in high-risk behaviours.

\section{Seroprevalence of HIV/HCV-coinfection among patients at a tertiary care center in Eastern India}

\author{
Prasad N ${ }^{1}$, Singh $\mathbf{N}^{2}$, Shahi S K $\mathbf{3}^{3}$ \\ Email ID for Correspondence: prasadnidhi72@gmail.com \\ ${ }^{1}$ Department of Virology, ${ }^{2}$ Department of Microbiology, IGIMS \\ Patna; ${ }^{3}$ Professor and HOD, Department of Microbiology, IGIMS \\ Patna
}

The human immunodeficiency virus (HIV) causes immunosuppression and if the patient is coinfected with Hepatitis C Virus (HCV), the probability of the person coinfected with HIV/HCV developing cirrhosis increases manifold (1). Studies on HIV/HCV-coinfected patients is highly recommended as it felicitates better counseling, treatment and follow up care of $\mathrm{HIV} / \mathrm{HCV}$-coinfected patients. Keeping this in mind we decided to do a study to determine the seroprevalence of $\mathrm{HIV} / \mathrm{HCV}$-coinfected patients in a tertiary care center in eastern India. A retrospective study from June 2017 to June 2018 was carried out in the virology lab of the tertiary care center of Eastern India in which records of all patients whose sera was screened for detection of Anti HCV antibody and HIV antibody by BenespheraMicrowell ELISA (IHF) were obtained and analyzed. A total of 37845 samples were screened during the above mentioned period out of which $142(0.37 \%)$ were found to be positive for HCV antibodies and $166(0.43 \%)$ were found to be positive for HIV antibodies, but none was found to be positive for HIV and HCV coinfection. Future studies with larger sample sizes are needed to estimate the seroprevalence of HIV/HCV-coinfection and to understand and address the risk factors associated with its acquisition.

\section{Ribavirin inhibits the Chandipura virus replication in Vero cells}

\author{
Anukumar B, Mun AB, Amalmol P \\ ICMR-National Institute of Virology (Kerala unit), Govt. T.D. \\ Medical College Hospital, Alappuzha, Kerala
}

Chandipura virus is an emerging tropical pathogen in India. The virus is reported to be associated with encephalitis syndrome in young children. The case fatality rate is around $55-75 \%$ and death ensues within $48 \mathrm{~h}$ of infection. Clinical management and symptomatic treatment is an only option available to treat the infected patients. Antivirals are preferable for exposed patients. As per the available literatures, no antivirals were reported for treatment of chandipura infection. This study aimed to test some antiviral drugs, which are available in the market and approved for human use, against chandipura virus. Seven antiviral drugs were screened for its effect on Chandipura virus replication in vero cells. Dose and time kinetics of drugs also studied. Differential expression of viral genes post treatment also explored in this study. Out of seven antiviral drugs tested against chandipura virus replication in vero cells, ribavirin showed some promising results. At $50 \mu \mathrm{g}$ extracellular concentration, ribavirin inhibited $50 \%$ of viral plaque formation in cells. Concentration dependent inhibition was noticed. More than $90 \%$ plaque reduction was observed at higher concentration $(150 \mu \mathrm{g})$ of ribavirin used in this study. The time dependent plaque reduction was also noticed in ribavirin treated cells. The drug was very effective when the cells were treated within an hour of post infection. Similarly, significant inhibition of plaque observed when the cells treated with ribavirin $4 \mathrm{~h}$ before infection. Ribavirin significantly inhibited the viral gene expression in infected cells. This study concludes that ribavirin effectively inhibits the chandipura virus replication and is a drug of choice for chandipura virus treatment. However, the detailed animal study is required before introducing into treatment for Chandipura virus infection.

\section{Proportion of Japanese Encephalitis, Dengue and Chikungunya virus infections among patients with acute febrile illness in a Tertiary care centre}

\author{
$\operatorname{SarkarA}^{1} *$, Majumdar $\mathrm{T}^{2}$, Debnath A, Majumder $\mathrm{S}^{4}$ \\ Email ID for Correspondence: apurbasarkar020@gmail.com
}

Arboviral diseases are on the rise, especially in the north-eastern region of India. After establishment of Viral Research and Diagnosis Laboratory in Tripura, initiative has been taken to diagnose the arboviruses Japanese Encephalitis (JEV), Dengue (DENV) and Chikungunya (CHIKV) by serology. Aim \& Objective: The aim of this study was to determine the proportion of JEV, DENV and CHIKV virus infections among patients with acute febrile illness in a tertiary care centre with objective 1 . To diagnose JEV, DENV and CHIKV by Immunoglobulin M capture enzymelinked immunosorbent assay (MAC - ELISA). 2. To determine the clinico-demographic profile of the study group. Materials and Methods: Duration of the study is three and half years (January, 2015 to June, 2018). Serum samples were collected from the patients with acute febrile illness and tested for JEV, DENV and CHIKV. MAC - ELISA was preformed as per the protocol of the manufacturer provided by NIV, Pune. Results: Out of 3357 subjects 32\% (1063) were MAC - ELISA positive. Among the viruses JEV predominated with $44 \%$ followed by DENV (31\%) and CHIKV (25\%). Moreover year wise percentage of positivity shows a steady increase in all the three viruses. JEV and DENV are prevalent in males with $62 \%$ and $64 \%$ over females i.e. $38 \%$ and $36 \%$ respectively. However CHIKV is predominated in females with $57 \%$, followed by males $(43 \%)$. In rural areas JEV positivity percentage is $43 \%$, which is more than DENV (32\%) and CHIKV (25\%). While in urban areas DENV is leading with $47 \%$ than JEV (28\%) and CHIK (25\%). Statistical Analysis: Graph pad prism 7 data analysis package was used to analyse the data. Conclusion: This study highlighted the importance of continuous surveillance for circulating Arboviral agents with molecular typing to look for the circulating genotypes/serotypes effective management and prevention. 


\section{Evaluation of the Indian TrueNAT micro Real-Time PCR device for case detection of High Risk Human Papilloma Virus (HR HPV) for cervical cancer screening in India}

\section{Singh P*, Negi SS, Bhargava A}

Department of Microbiology, AIIMS, Raipur, Chhattisgarh, India

High risk Human Papillomavirus particularly HPV type 16 and 18 play a cardinal role in the etiology of the cervical cancer. Truenat TM HPV-HR, a chip-based rapid Real-Time Polymerase chain reaction test requiring less than an hour in DNA extraction and amplification of specific E6 \& E7 gene for the semiquantitative detection of high risk HPV type 16,18,31,45 in female cervical specimens was evaluated for its diagnostic efficiency. 50 cervical intraepithelial neoplasia grade 2 or worse (CIN2) cervical specimens along with 10 Negative control samples were processed by TruenatTM HPV HR system targeting E6 \& E7 gene, and compared against standard GP5/GP6 PCR targeting L1 gene. All the 50 CIN grade 2 or worse samples were found positive for HPV by GP5/GP6 PCR. TruenatTM HR HPV showed a sensitivity of $86 \%$ (43/50) for CIN2+, specificity found $100 \%(10 / 10)$ and $100 \%$ positive predictive value and $58 \%$ Negative predictive value. $68 \%(34 / 50)$ samples showed HPV genotype 16/31 and $18 \%(9 / 50)$ belonged to HPV genotype 18/45. Laboratory test have a key role in preventing HPV driven carcinomas and guiding therapeutic interventions. TruenatTM Real-Time Micro PCR system being semi-automated technique provide rapid and effective means to identify HPV genotype.

\section{Recent Nipah Outbreak and global preparedness: Need of One Health approach}

\author{
Kumar $\mathbf{S}^{1}$, Saxena $\mathbf{S}^{2}$ \\ ${ }^{1}$ Center for Advanced Research (CFAR)-Stem Cell/Cell Culture Unit, \\ King George's Medical University (KGMU), Lucknow, India; ${ }^{2}$ CSIR- \\ Centre for Cellular and Molecular Biology, Uppal Road, Hyderabad, \\ India
}

Resurgence of Nipah virus (NiV) in South-East Asia has earned the global attention in last two decades. Approximately $>2$ billion of people are currently at risk of NiV infection. Recently NiV has struck again in India with at least $19 \mathrm{NiV}$ positive cases including 17 deaths and 753 suspected cases. Kozhikode, Kerala was the epicenter of the outbreak which appeared to be a localized occurrence of NiV. The emergence of $\mathrm{NiV}$ in India is speculated to be instigated by bats. The treatment is based only on the supportive care and needs isolation of the patients to reduce nosocomial infections. In order to cure, results from the antiviral perspectives are suggesting the most efficacious drugs are nucleoside based analogs which targets NiVRdRp. Most of the candidate vaccines for $\mathrm{NiV}$ are in the pre-clinical stages and have been tested in various animal models. The rationale of virus transmission from bats to animals or human has not been fully understood. Few studies are available in order to anticipate the mode of transmission and risk management strategies to prevent the emergence of $\mathrm{NiV}$ in future. Moreover, all the preceding outbreaks of NiV in South-East Asia have been reported in between month of December and May. The seasonal pattern of outbreaks might be correlated with the breeding season bats which may results in increased virus shedding and date palm sap harvesting period. Geographically, about two billion people subsist in vicinity inhabited by the Pteropus bats. Small, containable and isolated outbreaks have always a possibility of large epidemics in future, as we have learned from the recent outbreaks of Ebola and therefore we need to proceed with a sense of urgency in this regards.

\section{Molecular characterization of the common viral etiological agents causing acute diarrhea among children up to 5 years in a tertiary care setup of Tripura}

\author{
Ankurita Bhowmik ${ }^{1}$, Tapan Majumdar ${ }^{2}$ \\ ${ }^{1}$ Agartala Government Medical College (AGMC), Agartala, Tripura; \\ ${ }^{2}$ Associate Professor, Department of Microbiology, Agartala \\ Government Medical College (AGMC), Agartala, West Tripura
}

Acute viral gastroenteritis is one of the leading cause of mortality among children below 5 years worldwide. In India the mortality rate is $13 \%$ per year. The most notable viral agents causing diarrhoea are Rota virus, Adenovirus, Astrovirus, Sapovirus and Norovirus. This study was done to find out the circulating viral strains in Tripura. The aim of this study was to determine the role of viruses attributing to the cause of diarrhea among children $<5$ years of age with the following objectives; a) To estiate the proportion of diarrhoea attributable to viral cause, b) Socio-demographic profile of the study subjects and c) Genotypic characterization of the Rota viral agents. Samples were collected with proper labelling in a sterile container from cases during the acute illness. All the samples were tested for presence of rota virus antigen by ELISA (ROTACLONE) as per the manufacturer protocol. All the samples were stored at $-80{ }^{\circ} \mathrm{C}$ for molecular analysis by PCR. A total of 505 stool samples were collected from January, 2017 to July,2018, out of which 185 (27\%) samples were positive for Rotavirus, $0-1$ year of age being the highly prevalent age group and the overall prevalence was higher among the males than females. All though the infection was encountered throughout the year, there was an elevation in the diarrhoeal cases in the months of December to March. The most common genotypes of Gr-A rotavirus observed were G3P[8] followed by G1P[8], comprising of $62.9 \%$ and $31.45 \%$ respectively. Some other combinations of $\mathrm{G}$ and $\mathrm{P}$ serotypes were also observed such asG2P[8],G1P[4],G9P[8],G8P[10] along with some untyped strains. Out of 320 Rota ELISA negative samples, 93 samples were subjected to molecular screening that showed Adenovirus $(21 \%)$,Sapovirus $(6 \%)$, Norovirus (5\%) and Astrovirus (3\%). To the best of our knowledge this is the first molecular study of human rotavirus, adenovirus, norovirus, sapovirus and astrovirus in North East India. This study calls for a continuous surveillance and comprehensive diarrhoeal disease control strategy to reduce the burden of diarrhoea among children in Tripura, NE India.

\section{In-silico 3-D structural prediction and characterization of Hepatitis E Virus 'X-Domain'}

\section{Thakur V, Ratho R. K}

Email ID for Correspondence: rathopgi@yahoo.com Department of Virology, PGIMER, Chandigarh India

The macro domain $(\mathrm{X})$ is found to be ubiquitously present from Bacteria to humans and in many positive-strand RNA viruses like Rubella, Sindbis and SARS CoV. So far, HEV ORF1 X domain is known to interact with cellular ADP-ribose protein (involved in host pathogenesis). However, the detailed physiochemical characterization and putative refined structure of HEV X-domain with ligand binding active sites is not reported yet. So we proposed in silico 3-D structure 
and functional characterization of HEV X-domain which will significantly improve our understanding of $\mathrm{HEV}$ pathogenesis and replication. HEV X-domain sequence was retrieved from NCBI and characterized by ExPASY server. Crystallization probability was predicted by XtalPred, solvent accessibility by Raptor-X and disulphide linkages was predicted by DiANNA and DISULFIND server. Secondary structure was predicted by PredictProtein, SOPMA, PROFsec and Raptor-X Property server. 3-D structure was predicted by Phyre2, SwissModel, ITASSER and RaptorX and refined by ModRefiner server. Refined structure was validated by SAVES, RAMPAGE, QMEAN, Verify3D and ERRAT server. Finally the model was visualized in PyMol and the active binding site and ligands were predicted using RaptorX Binding tool and 3D ligandSite server. The predicted HEV $\mathrm{X}$ domain model was found to be more stable $\left(S^{2}>0.8\right)$, ordered and compact. HEV X-domain represented high crystallization probability (score 1), two disulfide bond linkages (Cys16-Cys145 and Cys34-Cys91), higher percentage of alpha helical (34\%) and extended strand providing thermodynamically stable nature. RaptorX predicted 3D structure and identified HEV X-domain as putative phosphatase (resemblance with 1spvA). Refined structure with $98.1 \%$ residues in favoured region (Ramachandran plot), verified with Verify3D $(85.44 \%$ residues 3D/1D score $\geq 0.2)$ server was acceptable predicted HEV X-domain. Multiplicity of 51 represented a deep binding pocket with 19 binding.residues for three different ligands viz. MES, APR and AR6. Physiochemical properties suggested that the model is stable and in ordered form and has higher probability for crystallization which could be tried experimentally. Three ligands are predicted to bind with active binding site of HEV $\mathrm{X}$-domain might prove to be a potential inhibitor to limit the HEV pathogenesis.

\section{Varicella outbreak in a residential child care institution in North India - Time to revisit Immunization guidelines under the Juvenile Justice Act 2015}

\section{Aditya Sood, Bhavneet Bharti and Mini P Singh}

Varicella outbreaks are common in India as varicella vaccine is not included in the Universal Immunization schedule for India. Also, a low seroprevalence of varicella antibodies has been reported among Indian children (Balraj and John 1994, Lokeshwar et al. 2000, Singh et al. 2015, Singh et al. 2011). The outbreaks are common in residential child care settings where children are in close contact with each other leading to increased transmission among peer groups. Outbreak occurred in a govt owned residential child care institution with 103 children. Case of varicella was defined as a child with a macula-papulovesicular rash without any apparent cause in children residing in the centre. Swabs collected from the easily accessible vesicular lesion in two affected children were found to be positive for VZV-DNA. We line listed the cases and collected detailed clinical information on age, sex, date of onset, number of lesions (severity), treatment taken, number of days school missed and out of pocket expenditure. Vaccination of all the susceptible children in addition to isolation of cases, quarantine of suspects and proper screening for new cases was the major control strategy adopted. However necessary sanctions and permissions took time resulting in delay in the outbreak response. Qualitative data collection included narrative information related to the problems faced by caregivers in management of these children. Out of the 103 children we identified around 30 cases of varicella. The index case was first identified on 27th January 2018. However it was only in April when the number of cases peaked that the vaccination as control strategy was initiated. All the children presented with rash. Severity of the varicella as measured by the number of skin lesions $<50(\mathrm{n}=10), 50-100(\mathrm{n}=5), 100-150$ $(\mathrm{n}=15)$. No child however required hospitalization. All children missed the school for 2 to 3 weeks. The burden of care was significantly increased for the caretakers and isolation policy was difficult to maintain in the presence of common washroom facility. $83 \%$ of the children received Acyclovir treatment and their mean cost of treatment was Rs $597 \pm 228$. Residential care settings are emerging as susceptible niche area for rapid spread of varicella infection with high cost of medical care. Keeping in mind the burden of care of these children, we propose mandatory inclusion of Varicella vaccination under the Juvenile Justice Act 2015 for children in need of care and protection in residential child care settings.

\section{Rapid decline of anti-measles antibodies in a cohort of infants from birth to 12 months of age, calls for an urgent change in vaccination strategy in India}

Laxmi Makam, Joseph L. Mathew, Radha Kanta Ratho, Mini P. Singh, Sourabh Dutta, Bhavneet Bharti, Vanita Suri, Deepika Rechal Massey

\section{Departments of Pediatrics and Virology, PGIMER Chandigarh}

Since 1985, India has been administering a single dose of measles vaccine to all infants at 9 months of age. This age was chosen to balance the disappearance of maternal (transplacental) antibodies with the increasing risk of developing measles. Measles infection occurs before the age of vaccination in 10-15\% cases, suggesting the absence of immunity well before the age of vaccination. This study was designed to estimate the level of measles specific IgG antibodies in a cohort of term infants followed from birth to 12 months of age; and the pattern of antibody decline in them.

We enrolled a cohort of 168 term infants in a prospective longitudinal study and measured serum IgG anti measles antibody levels (AMAL) at birth, 3 months, 6 months, 9 month (pre-vaccination) and 12 months of age. Maternal serum AMAL was also measured at the time of delivery. Antibody levels $>12 \mathrm{U} / \mathrm{ml}$ were considered protective. The median (IQR) maternal AMAL at delivery was 66.15 (26.23 to 138.80) and the level in infants at birth was 66.83 (29.04-128.79). At birth, 142/168 (84.5\%) mothers and 149/168 $(88.7 \%)$ infants had protective levels. The proportion of protected infants was $25 / 90(27.8 \%)$ at 3 months, $2 / 84(2.38 \%)$ at 6 months and $1 / 34(2.90 \%)$ at 9 months (pre-vaccination). 3 months after vaccination, $81.2 \%$ infants had protective levels of antibodies whereas $18.8 \%$ did not.Infant protection at birth correlated with maternal protection status (Spearman's correlation coefficient $0.849, p=0.0001$ ). Similarly, protection at 3 months of age correlated with birth antibody level (Spearman's correlation coefficient 0.642, $p=0.0001$ ). Measles IgG antibody levels rapidly decline after birth and reach levels far below protective level well before 9 months of age. The majority of infants are susceptible as early as 3 months of age, while all are susceptible by 6 months of age. This requires consideration of earlier vaccination to protect these infants, which could have significant public health implications. 
Prevalence of viral hepatitis in multitransfused thalassemic population of Haryana: a seven-year retrospective study

\section{Lall S, Gill PS, Gupta T, Rathee P, Chaudhary U}

Email ID for Correspondence: sujatamed@gmail.com Department of Microbiology, VRDL lab, PGIMS Rohtak, Haryana

Thalassemia is one of the common genetic diseases in the world.Thoughregular blood transfusion improvesthe overall survival of patients; it carries a definite risk of acquisition of transfusion transmitted infections. The present study was done to assess the prevalence of viral hepatitis in multitransfused patients of thalassemia major at our centre. Prospective analysis of retrospectively maintained database of 227patients of thalassemia major receiving transfusion at our institute was done over a period of 7 years from Jan 2011-Jan 2018. Demographic details of thepatients were noted. Results of periodic screening of their sera for hepatitis B surface antigen ( $\mathrm{HBsAg}$ ) and $\mathrm{HCV}$ antibody ( $\mathrm{HCV} \mathrm{Ab}$ ) by ELISA were analyzed. The study sample consisted of 165 boys and 62girls suffering from thalassemia major and receiving blood transfusion (BT) at our centre. Most of these patients had received BT from other hospitals also before enrolling at our Centre. The median age was 12 years. Twenty (8.8\%) patients (14boys and 6 girls) were $\mathrm{HCV} \mathrm{Ab}$ positive by ELISA and one $(0.44 \%)$ was HBsAg positive. Out of 20 $\mathrm{HCV} \mathrm{Ab}$ positive patients, three were also HBsAg positive by ELISA (coinfection rate 1.32\%). The prevalence of acquisition of $\mathrm{HCV}$ and HBV infection was directly related to number of blood transfusions received by the patients. The prevalence was significantly less compared to blood samples before $2014(p<0.001)$. A large number of $\mathrm{HCV}$ infected patients remain undiagnosed clinically due to the absence of symptoms specific to hepatic injury like jaundice. Also use of screening tests of blood in blood banks based on antibody detection in infections with high incubation periodmay eventually lead to iatrogenic transmission of such infections to patients receiving BT.Thus there is a compelling need to implement the policy of nucleic acid based screening tests in blood banks to reduce transmission of BTrelated viral infections.

\section{Clinico-epidemiological profile of respiratory syncytial virus (RSV) in children less than five years of age: first report from Central India}

\author{
Nema $S^{1}$, Nema $R K^{1}$, Bhatt $G^{2}$, Shrivastava $V^{2}$, Kudsia $A^{1}$, \\ Raghuwanshi $\mathrm{A}^{1}$, Malik $\mathrm{S}^{2}$, Biswas $\mathrm{D}^{1}$
}

Email ID for Correspondence:

snema.microbiology@aiimsbhopal.edu.in

Deptt. of Microbiology ${ }^{1}$,Deptt. Of Paediatrics ${ }^{2}$, AIIMS Saket Nagar, Bhopal, India

RSV is the most common cause of bronchiolitis and pneumonia in young children in both the community and hospital setting and is associated with high mortality and morbidity in under five children. Ongoing surveillance of the clinical and epidemiological parameters of RSV is important for development of preventive and management strategies. In view of scarcity of data in central India, the present study was planned to assess the clinical and epidemiological profile of RSV infection in children $<5$ years of age in a tertiary care hospital. This is an ongoing study which included children $<5$ years of age who visited our hospital with complaints of lower respiratory tract infection. Nasopharyngeal aspirates were collected and transported to microbiology department in viral transport media. The specimens were processed for RSV reverse-transcriptase polymerase chain reaction. Severity of respiratory tract infection was evaluated by PRESS score in RSV positive cases. A total of 30 children were recruited in the study [Till Date]. RSV was detected in $9(30 \%)$ cases. $8 \mathrm{RSV}$ positive cases were male and 5 were $<1$ year of age. Respiratory infections due to RSV were categorised as mild, moderate and severe in 3, 4 and 2 cases respectively. Results till November 2018 will be presented during the conference. Initial findings of our study demonstrate a prevalence of approximately $30 \%$ for RSV infection in under five children with manifestations of lower respiratory tract infection.

\section{Seasonality trends of rotavirus associated diarrhea in Indian children $<5$ years of age in a multisite hospital based surveillance study; 2012-2016}

\section{Karthick.J, Archana Sriraman, Sidhartha Giri, Gagandeep Kang}

Email ID for Correspondence: karkeyan1987@gmail.com Department \& Institution: Wellcome Trust Research Laboratory, Christian Medical College, Vellore

Rotavirus is the leading cause of severe diarrhea in young children $<5$ years of age worldwide, and causes approximately 78,000 deaths in Indian children annually.

Aim: To assess the seasonality trends of Group A rotavirus strains in children $<5$ years of age in a four year multisite hospital based surveillance study from 2012 to 2016.

Stool samples were collected from children $<5$ years hospitalized with diarrhea from seven sites across India during 2012-2016, which included 5 southern (Vellore, Trichy, Kolenchery, Hyderabad, Tirupati) and 2 northern (Delhi, Ludhiana) sites. Samples were collected after obtaining informed consent from parents/guardian. All stool samples were screened for group A rotavirus by ELISA (Rotaclone). A total of 5834 samples were collected during the time period of four years (2012-2016) from seven sites. 2069 (35.4\%) samples were positive for group A rotavirus by ELISA (Rotaclone). The proportion of rotavirus associated diarrhea increased during November to February of each year across the northern and southern sites. However, there was a difference in the proportion of rotavirus diarrhea between the two regions during the peak months (range: $41.2-65.5 \%$ in the north, $31.7-54.9 \%$ in the south). Although rotavirus associated diarrhea was seen throughout the year, we found that that the proportion of rotavirus diarrheal episodes was more during the colder months of the year (November-February), and this trend was seen in north as well as south Indian sites.

\section{RNA-Seq analyses of short- and long-term antiretroviral treated patients with Kaposi's sarcoma}

\section{Rajput A, Kumar Archit ${ }^{1}$ and Kumar $\mathbf{M}^{1}$}

Email ID for Correspondence: manojk@imtech.res.in Virology Discovery Unit and Bioinformatics Centre ${ }^{1}$, Institute of Microbial Technology, Council of Scientific and Industrial Research (CSIR), Sector 39A, Chandigarh, India

Kaposi's Sarcoma-associated herpes virus (KSHV) is a member of Herperviridae and known to cause Kaposi's sarcoma (KS). A recent study by Tso et al. performed the differential expression gene (DEG) analysis. They utilized DESeq2 method using hg19 version of human 
genome to compare KS lesions against normal tissues among four patients i.e. p22, p23, p32, and p83. However, they haven't explored the effect of short-term (2-3 months, p22 \& p23) and long-term ( $>2$ years, p32 \& p83) antiretroviral (ART) treatment. In the present study, we have investigated the DEG pattern among above two groups of patients. We performed RNA-Seq analysis using tophat $2 /$ cufflink pipeline against latest human reference genome hg38. The DEG profiling was carried out through cuffdiff method between two categories and mapped on GO processes, and KEGG pathways. On aligning the transcriptomic data of KS patients with the human and KSHV genomes (hg38 and NC_009333), we found maximum reads were aligned against human genome, with at least $91.20 \%$ and $86.70 \%$ alignment rate for lesion and control tissues, respectively. Whereas we observed more reads aligned with KSHV genome from KS lesion (ranges from 158 to 1285 reads) as compared to normal tissues (0-36 reads). Further, the DEG analyses (cutoff \pm 1.5 fold) revealed that most of the differentially regulated genes $(762 \mathrm{Up}+$ 1093Down) during the short-term treatment, primarily involved in extracellular matrix and metabolic processes were stabilized after long-term treatment. Contrary, because of the long-term treatment, the genes involved in cell cytoskeleton become up-regulated and the genes for the hair cycle and keratin filament formation become downregulated. These findings provide the regulation of the genes during long-term ART in KS patients, which could assist in understanding the role of host-factors in disease biology and may be helpful in diagnostics or therapeutic interventions.

\section{Computational prediction of integrase targeting antiviral peptides}

\section{Bhardwaj A, Patel PK, Rajput A, and Kumar M}

Email ID for Correspondence: pradeep@imtech.res.in

During the past three decades world has seen many viral diseases outbreaks these include Zika, Ebola, Influenza, Dengue etc. These viral diseases are emerged as a great threat to public health due to shortage of the effective therapeutics against viruses. Peptides based therapeutics are very promising due to their low toxicity in the host and hence many are in various stages clinical evaluation. Antiviral peptides can target various stages of the viral life cycle including integration of viral genome to host cell.

In the present study, we developed a prediction method using 225 experimentally validated peptides targeting integrase from "AVPdb" and "HIPdb" repositories. The prediction models were developed using supervised learning by support vector machine through five-fold cross validation approach. The overall data set includes 76 highly effective and 127 least or non effective peptides. We used various peptide features like amino acid composition, dipeptide composition, five amino acid positions at $\mathrm{N}$ and $\mathrm{C}$ terminals, and their hybrids. The overall dataset was sub-divided into training/testing T203(76p + $127 n)$ and independent validation V22 $(14 p+8 n)$ data sets for performing internal and external cross-validations. Best performing hybrid model achieved sensitivity, specificity, accuracy, Matthews' correlation coefficient and receiver operating characteristics of $63.16 \%, 73.23 \%, 69.46 \%, 0.36,0.3$ for training/testing while $78.57 \%$, $62.50 \%, 72.73 \%, 0.41,0.74$ for independent validation datasets respectively. We have also observed differences in the amino acids preferences between effective and non-effective peptides. This in silico method would be helpful for prediction of viral integrase inhibitng peptides.
Prediction and analysis of viral and bacterial MHC class II immunogenic and non-immunogenic epitopes restricted to human host

\section{Mohd. Shoaib Khan, Amit Kumar Gupta and Manoj Kumar}

Email ID for Correspondence: manojk@imtech.res.in Virology Discovery Unit and Bioinformatics Centre, Institute of Microbial Technology, Council of Scientific and Industrial Research (CSIR), Sector 39A, Chandigarh, India

Identification and designing of better immunogenic epitopes is crucial for effective vaccine development and remains an important area in the field of immunoinformatics. There are attempts to predict the binding affinity of peptides to MHC class II molecules. However, very few attempts were made to predict the immunogenecity of the peptides and there is a need and scope for better algorithm or organism specific immunogenicity predictor. In this, MHC class II immunogenic (positiveP) and non-immunogenic (negativeN) epitopes belonging to virusesvir (P7181 and N4675) and bacteriabac (P2907 and N2329) specific to human host were retrieved from the IEDB v3.10.0. Further 15-mer epitope subset datasets were also prepared to develop prediction models. We have employed Support Vector Machine (SVM) to develop prediction model using different datasets i.e. $\quad$ vir $(\mathrm{P} 7181+\mathrm{N} 4675)$, bac $(\mathrm{P} 2907+\mathrm{N} 2329)$, vir-vs-bac $($ P7181 + N2907), vir_15mer (P2416 + N2409), bac_15mer (P931 + N1289), vir-vs-bac_15mer (P2416 + N931). These datasets were divided into training/testing $(\sim 80 \%)$ and validation $(\sim 20 \%)$ dataset. Training/testing datasets $(\sim 80 \%)$ were used for the fivefold cross validation to develop predictive models employing different sequence features viz. amino acid composition (AAC), di-peptide composition (DPC), AAC + DPC hybrid, binary (BIN) and AAC + DPC + BIN hybrid. Validation dataset $(\sim 20 \%)$ is used for the independent performance validation. During fivefold cross-validation on training/testing datasets, SVM achieved the maximum accuracy, Matthew's correlation coefficient (MCC) and Receiver operating characteristic (ROC) of $67.1 \%, 0.33,0.72$ for $\operatorname{vir}(\mathrm{P} 7181+\mathrm{N} 4675) ; 77.58 \%, 0.55,0.85$ for $\operatorname{bac}(\mathrm{P} 2907+\mathrm{N} 2329)$; $86.39 \%, 0.69,0.93$ for vir-vs-bac(P7181 + N2907); 68.16\%, 0.36, 0.73 for vir_15mer $(\mathrm{P} 2416+\mathrm{N} 2409) ; 75.84 \%, 0.51,0.82$ for bac_15mer(P931 + N1289); 85.84\%, 0.66, 0.93 for vir-vs-bac_15mer(P2416 + N931) respectively. All developed models performed equally well on their respective independent validation datasets. We have developed improved MHC class II epitope prediction method and also model to differentiate virus vs bacterial immmunogenic epitopes specific to human host. We hope this would help in identifying organism specific immunogenic epitopes as putative vaccine candidate.

\section{Computational search for antiviral drugs against emerging Nipah virus}

\section{Vinay Randhawa, Shivalika Pathania\#, and Manoj Kumar}

Email ID for Correspondence: manojk@imtech.res.in Virology Discovery Unit and Bioinformatics Centre, CSIR-Institute of Microbial Technology (IMTECH), Chandigarh India

Nipah virus (NiV) is member of the genus Henipavirus in the family Paramyxoviridae, which is considered as an emerging deadly virus due to its pathogenicity, has caused several outbreaks in Asian countries. While no therapeutics are currently approved against this virus, we aimed to prospect for small molecule antagonists against $\mathrm{NiV}$. We considered $\mathrm{NiV}$-nucleoprotein $(\mathrm{NiV}-\mathrm{N})$ as potential target 
based on its prime role in transcription and replication of viral mRNA and genomic RNA. NiV-N structure was obtained from PDB database (4CO6) and a library of FDA-approved drugs from DrugBank. After quality assessment and pre-processing, information of interactions between ligands and receptor were obtained by docking-based virtual screening. As a post-docking filter, only those ligands that occupy binding pocket and interacting with functionally important residues, inferred on the basis of 3D structure overlap with Respiratory syncytial virus ribonucleoprotein, were considered. After molecular docking and assessment of toxicity risks and drug-relevant properties, several potential candidates for $\mathrm{NiV}$ treatment were identified such as Bromocriptine and Paritaprevir, and Simeprevir, which are already reported for their antiviral activity against Zika Virus and Hepatitis $\mathrm{C}$ Virus, respectively. Importantly, this analysis also provided some novel candidates against NiV-N. Structure-based clustering indicated identified molecules to be structurally very distinct, except for Simeprevir and Paritaprevir that were present in one common group. Our analysis identified FDA approved drugs as potential inhibitors of Nipah Virus and we expect these leads to be successful in further experimental evaluation.

\section{Etiology of viral hemorrhagic fever in UP West in tertiary care hospital}

\section{Teerathanker Mahaveer University in Moradabad Delhi road,TMMC \& RC, Moradabad, India}

Viral hemorrhagic fever (VHF) is characterized by severe febrile illness along with hemorrhagic manifestations. These infections can progress to high fever, multi-organ failure, shock and death in many cases.Many viruses cause viral hemorrhagic fever but Dengue and Chikunguniya virus are more commonly seen in India however the exact prevalence of these infections is not known in UP West is not known. This study was aimed to assess the prevalence of Dengue and Chikungunya virus in UP West. Cases presenting with acute onset of fever with with two or more of the following symptoms including nausea, vomiting, abdominal pain, dirrhoea, weakness, skin rash, headache, lesions on soft palate, unexplained bleeding from any site or joint pain were included in the study. Serum sample from these cases were tested for Dengue virus IgM antibody, Dengue NS1 Antigen, Chikunguniya virus IgM by ELISA. A total 200 cases were enrolled of which a definite etiology was confirmed in 85 cases. Out of the 85 positive cases, 28 cases were reactive for Dengue NS1 Antigen, 46 were reactive for Chikunguniya virus. Males were more commonly affected as compared to females (69\% vs. $31 \%$ ). Dengue virus is the most common cause of VHF in west UP India followed by Chikunguniya virus. Both Dengue and Chikunguniya virus are included in NVBDCP (National vector borne disease control program). Regular surveillance, monitoring and preventive measures are required to curtail these diseases thereby reducing the mortality and morbidity associated with these diseases.

\section{Frequency of Candida infection in HIV patients and its antifungal susceptibility in TMMC \& RC Moradabad}

\section{Farha, Dr.Shewta R. Sharma, Dr. Umar Farooq}

AIDS (Acquired Immunodeficiency Syndrome) is a syndrome caused by a virus called HIV (human Immunodeficiency virus). The disease alters the immune system, making people more vulnerable to infections and diseases. Candida is an opportunistic fungal pathogen in patients with HIV. Candidiasis commonly affect the skin and mucus membrane. Its incidence significantly worldwide. Identification of Candida isolates up to the species level is important. There is increased resistance to antifungal agents and some species are intrinsically resistant to azoles. The present study was undertaken to describe the various Candida species causing Candidiasis in HIV patients and its antifungal susceptibility among all age groups. A total 26 Candida isolates were obtained from all age groups population in HIV positive samples. These isolates were identified on the basis of Gram Staining. SDA, CHROM Agar, Germ tube test. Out of 32 HIV positive patients 26 were positive for Candida infection in which 18 were Candida albicans and 8 were non Candida albicans (NAC). The isolated Candida species were highly sensitive to amphotericin B, Nystatin, Voriconazole and resistant to Fluconazole. Candida albicans was the most prvalant organism isolated from the HIV patients in tertiary care hospital. Variation in susceptibility pattern to antifungal agents were seen.

\section{Differential gene expression pattern of host immune genes in Japanese encephalitis infected patients}

\section{Purvita Chowdhury, Siraj A. Khan}

Email ID for Correspondence: sirajkhanicmr@gmail.com Arbovirology Division, ICMR-Regional Medical Research Centre, NE Region, Dibrugarh, Assam, India

One of the deadliest viral encephalitis caused by Japanese encephalitis (JE) virus shows clinical manifestations ranging from asymptomatic to severe neurological symptoms and even death. The precise pathophysiology for the diverse clinical spectrum of JE has not yet been defined. Studies have postulated that during JE infection, inflammatory cytokines and chemokines are produced after the initial recognition of viral antigens through the engagement of TLR pathways. However, there is paucity of knowledge on the expression levels of chemokines and TLRs among mild and severely affected JE patients. Therefore, the present study aims to understand the expression pattern of chemokines, chemokine receptors and TLRs among mild and severe JE cases. Gene expression levels of chemokines (CCL2, CCL5), its respective co-receptors (CCR2, CCR5) and TLRs (TLR 3, TLR7, TLR8 and TLR9) was assessed in peripheral blood mononuclear cells (PBMCs) among mild and severe JE patients as well as in healthy individuals. RNA extraction and cDNA synthesis was performed as per the manufacturer's guidelines. mRNA expression levels were quantified using real-time PCR. Our study showed significant down-regulation of chemokines (CCL2, CCL5), their co-receptors (CCR2, CCR5) and TLR3 in mild JE cases as compared to controls. Significant difference of gene expression was observed among mild and severe JE cases for CCL2 $(p<0.001)$, CCL5 $(p<0.01)$ and TLR7 $(p<0.05)$.

In conclusion, our results suggest that the variation in gene expression of chemokine CCL2, CCL5 and TLR7 is associated with JE severity and may be used as a probable marker for JE severe pathogenesis. 
Hepatitis B core antibody positive liver grafts can be safely given in core antibody positive and negative recipients with thorough virological evaluation and monitoring

Dr. Nitin Kumbhar' ${ }^{1}$ Dr. Reshu Agarwal ${ }^{1}$, Dr Ashok Choudhury ${ }^{2}$, Dr Viniyendra Pamecha ${ }^{3}$, Dr. Ekta Gupta ${ }^{1}$

Department of Clinical Virology ${ }^{1}{ }^{2}$ Department of Hepatology; Department of Hepato-Pancreato-Biliary Surgery ${ }^{3}$, Institute of Liver and Biliary Sciences, New Delhi

Hepatitis B core antibody ( $\mathrm{HBcAb})$ is a marker of exposure to the virus. The use of $\mathrm{HBcAb}$ positive grafts for liver transplantation has potential to expand the donor pool especially in hepatitis B endemic areas. In this retrospective study, medical records of consecutive living donor liver transplant recipients from January 2015 to August 2018 were analysed for HBV markers (HBsAg, HBcAb, HBV-DNA) in both donors and recipients and correlated with post-transplant occurrence of HBV infection in recipients. During the study period, 311 liver transplants were conducted. $\mathrm{HBcAb}$ was found to be positive in $9.6 \%$ of the donors $(30 / 311)$. These $30 \mathrm{HBcAb}+$ ve liver grafts were transplanted to $15 \mathrm{HBcAb}$ positive (4 anti-HBs +ve; 11 anti$\mathrm{HBs}-\mathrm{ve}$ ) recipients and $15 \mathrm{HBcAb}$ negative (5 anti-HBs +ve, 10 anti$\mathrm{HBs}-\mathrm{ve}$ ) recipients. In $\mathrm{HBcAb}+\mathrm{ve}$ group none of the recipients developed $\mathrm{HBV}$ infection during the follow up period (mean 9 months; range 2-22 months). In HBcAb -ve anti-HBs +ve group no recipient developed HBV infection (mean follow up 10.2 months; range: 2-32 months). However, in the $\mathrm{HBcAb}$-ve anti-HBs -ve group one recipient $(1 / 30 ; 3.33 \%)$ developed HBV infection documented at 32 months after transplantation. HBV DNA was $6.8 \times 10^{7}$ $\mathrm{IU} / \mathrm{mL}$.

The sole post-transplant HBV infection was seen in $\mathrm{HBcAb}$ negative group and the recipient had pre-transplant anti-HBs titer of $<10$ $\mathrm{mIU} / \mathrm{mL}$; below the protective levels recommended. HBcAb positive liver grafts can be safely given in core positive recipients and core negative recipients with protective anti-HBs titres. The pre-transplantation anti-HBs titre in recipients, especially $\mathrm{HBcAb}$ negative, is very critical and should be evaluated as anti-HBs defines protection against HBV infection. Careful virological monitoring is recommended in recipients who demonstrate low levels of anti-HBs titres and in non-responders. Further, anti-HBV prophylaxis can be recommended in select cases.

\section{Identification of potential candidates to design therapeutic vaccine against HPV 16 infection: molecular docking study}

\section{Kinikar Ma \& Kulkarni-Kale Uaa}

Email ID for Correspondence: urmila.kulkarni.kale@gmail.com Bioinformatics Centre, Savitribai Phule University of Campus, Ganeshkhind Road, Pune, India

Human papillomavirus (HPV) infection causes genital warts and anogenital, head and neck cancers. It is a sexually transmitted infection found in almost all sexually active individuals. HPVs are classified as high-risk (cancer inducing) and low-risk (cause genital warts). HPV type 16 is a high-risk HPV responsible for causing cervical cancer in $70 \%$ of women. Prophylactic vaccines are available against some types of infectious HPVs which have resulted in prevention of HPV infection. But, due to lack of awareness and vaccination, there are cases of women suffering from cervical cancer due to HPV type 16 infections. Hence, there is a need to develop therapeutic vaccine against HPV type 16 infections. The HPV 16 genome codes for 8 proteins (E1, E2, E4, E5, E6, E7, L1, and L2) out of which E6 are E7 are oncoproteins. Epitopes derived from oncogenic proteins could be considered potential targets for designing therapeutic vaccine. This study aims to identify such targets from E6 protein of HPV 16 using docking simulations. Experimentally validated T-cell epitopes of E6 were retrieved from IEDB online ( https://www.iedb.org/). These epitopes were docked onto MHC class I and MHC class II molecules using ZDOCK-RDOCK, Glide (Schrödinger software) and ClusPro. Analysis of the docked poses was done using Discovery Studio. Eight epitopes were docked onto HLA*A0201, HLA*B5703, HLA*B2709, HLA*DPA1*0103 MHC alleles based on their affinity to a particular allele.The molecular docking results showed significant interactions between the anchor residues of MHC class I molecules and epitopes. Therefore, epitopes were ranked on the basis of respective docking scores and experimental validation of CTL responses curated from publications. On the basis of the ranking, epitopes'22-TIHDIILECV-31' and'11KLPQLCTEL-19' are identified as potential targets for therapeutic vaccine design.

\section{Prevalence of cytomegalovirus infection in patients with inflammatory bowel disease at a tertiary referral centre: a 12 year retrospective study}

\section{Gupta $\mathbf{M}^{1}$, Dhole $\mathbf{T N}^{2}$}

Email ID for Correspondence: dreamsmaterialise@gmail.com Senior resident, Clinical Virology, Department of Microbiology ${ }^{1}$ Professor and head -Clinical Virology ${ }^{2}$ Sanjay Gandhi Post Graduate Institute of Medical Sciences, Lucknow

Cytomegalovirus (CMV) infection exacerbates IBD refractory to immunosuppressive therapies. Outcome of patients with IBD and CMV infection is likely to be worse, if treatment with immunosuppressive drugs is continued without treating the CMV infection. The current ECCO guidelines recommend colonic tissue PCR as the preferred test for screening for CMV colitis. We examined the diagnostic yield of PCR in suspected CMV infection from intestinal biopsies in IBD patients. Retrospective study wherein patients who underwent testing for $\mathrm{CMV}$ on intestinal biopsies were identified from January 2006-March 2018. Patients with serum CMV PCR or antibody testing were not included. From 2006 to 2018, at our institution $55.89 \%(109 / 195)$ of patients with IBD tested positive for CMV by tissue PCR. of PCR + patients, majority were on steroids either alone or in combination with immunomodulator and/or biologic therapy. CMV DNA was detected in inflamed colonic tissue $40.81 \%(20 / 49)$, rectal tissue $62.4 \%(78 / 125)$ sigmoid tissue $70 \%(7 / 10)$, rectosigmoid tissue $50 \%(1 / 2)$, ileal tissue $67 \%(2 / 3)$, duodenal tissue in $33.3 \%(1 /$ 3 ) and not detected in caecal tissue $(0 / 1)$. Year wise distribution of CMV-positive patients and frequency of testing was found to be markedly increasing over the years. CMV reactivation potentially causes severe colitis in these patients who are more likely to become hospitalized, have longer lengths of stay and higher mortality rates. Patients with CMV infection are more prone to develop steroid refractoriness and a cut-off value of CMV DNA to predict the steroid refractoriness is yet to be identified but taken as 250 copies $/ \mathrm{mg}$. Histology is quite specific but is of low sensitivity.Therefore, an early detection of CMV DNA in intestinal biopsy is of utmost importance for the correct and timely management of IBD patients with CMV infection and reduction of colectomy rates. 


\section{Characterization of E7 gene of HPV16 in Indian population: an evolutionary bioinformatics study}

\section{Email ID for Correspondence: sanket.limaye22@gmail.com}

Human papillomaviruses (HPV) belong to Papillomaviridae family. HPV are non-enveloped, circular DNA viruses with $7.9 \mathrm{kbp}$ genome encoding 7 genes. HPV are classified on the basis of its capsid protein L1 into 5 genera. HPV16, a member to Alphapapillomavirus 9, is considered a high risk type known to cause cervical cancer in women. E7 gene codes for an oncogenic protein which targets Retinoblastoma tumor suppressor $(\mathrm{Rb} 1)$ and cause cell proliferation. The study aimed at characterization of variations observed in E7 gene of HPV16 isolates circulating in Indian population. The sequencing of 125 isolates was carried out at National AIDS Research Institute (NARI). The sequences thus obtained were compared with 50 reference sequences of global isolates in public domain databases with an objective to understand extent of evolutionary forces operational in E7. Methodology: The bioinformatics pipeline included multiple sequence alignments, mutation detection, phylogenetic analysis (alignmentbased and alignment-free), mapping known antigenic sites and mutations on 3D structure. Results: The nucleotide sequences of E7 gene of Indian isolates showed 10 mutations of which 2 are nonsynonymous mutations. Phylogeny of E7 gene helped identify circulating lineages of HPV in Indian population. Majority of Indian isolates belonged to 'A' lineage. A total of 5 B-cell and 15 T-cell experimentally validated epitope of E7 were obtained from IEDB which were mapped onto the E7 protein structure. Conclusion: E7 gene undergoes a few mutations and depicts a clustering pattern similar to the lineages obtained using L1 gene, which is used as a marker for HPV classification. With no recombination and in the absence of positive and pervasive selection E7 gene is highly conserved. Of the two amino acid mutations found at positions 29 and 57 in Indian isolates, the mutation $\mathrm{F} 57 \mathrm{~V}$ is part of a T-cell epitope.

\section{Study of Chikungunya virus infection in the state of Meghalaya}

\section{Sapam $\mathrm{S}^{1}$, Phukan AC ${ }^{2}$, Prasad $\mathrm{AK}^{3}$}

Email ID for Correspondence: sanasapam31@gmail.com Department of Microbiology ${ }^{1,2,3}$, North Eastern Indira Gandhi Regional Institute of Health and Medical Sciences, Shillong, India

Chikungunya is becoming a major health problem in North-East India and its prevalence is gradually increasing in this part of the country. This study was undertaken to detect the prevalence of chikungunya in patients attending a tertiary care centre in North east India and also to study the demographic and clinical profiles of such patients. This was a 1 year study conducted from January 2017 to December 2017. Whole blood was the sample collected from suspected cases (as per the selection criteria mentioned in the National guidelines for Clinical Management of Chikungunya, 2016). Serum was separated from these samples and MAC ELISA was employed to detect antiChikungunya IgM antibody. The demographic and clinical profile of the patients was noted in details. Serum sample of 47 out of 307 suspected cases were confirmed to be suffering from Chikungunya. A whooping $66(21.5 \%)$ samples was found to be give equivocal results. These patients with equivocal results were asked to give repeat samples after 10 days but none of them followed up. Male $(57.45 \%)$ preponderance was seen among the infected patients where 21-30 years age group (29.7\%) was found to be predominantly affected. Majority of the cases were from Meghalaya (93.6\%) followed by the adjoining state of Assam (4.25\%). The most affected district in Meghalaya was West Garo Hills (93.1\%). Most of the cases presented within 1-5 days since the onset of symptoms $(78.72 \%)$. Among the confirmed cases fever $(85.10 \%)$, myalgia $(72.34 \%)$, polyarthralgia $(31 \%)$, headache $(46.80 \%)$ and skin rashes $(4.25 \%)$ were the major clinical findings. Information generated from this study may be used for further study in depth the molecular characterisation of the circulating strains of the viruses in the NE India. Further the study will be useful to detect any new emerging endemic area of chikungunya and to adopt preventive strategies in districts where Chikungunya cases are reported.

\section{Study of Japanese encephalitis viral infection among the patients attending tertiary care centre in North-east India}

\section{Biswas $\mathrm{D}^{1}$, Phukan $\mathrm{AC}^{2}$, Prasad $\mathrm{AK}^{3}$}

Email ID for Correspondence: drdeepayanbiswas@gmail.com Department of Microbiology ${ }^{1,2,3}$, North Eastern Indira Gandhi Regional Institute of Health and Medical Sciences, Shillong, India

Acute encephalitis syndrome caused by Japanese Encephalitis (JE) is a major public health problem of North East (NE) India. The present study was undertaken with the objective to study the prevalence of Japanese Encephalitis among the suspected patients attending tertiary health care centre in NE India and also to study the demographic and clinical profile of such patients. Cerebrospinal fluid (CSF) and serum samples were collected from 357 suspected acute encephalitis patients from September 2016-August 2018. CSF was collected from 233 patients, serum from 66 patients and both CSF \& serum was obtained from 58 patients. These samples were subjected for detecting anti-JE IgM antibody employing IgM antibody capture ELISA (MACELISA) supplied by National Institute of Virology (ICMR), Pune. The epidemiological and clinical profile of the patients were noted in detail. In a total of 357 suspected acute encephalitis patients 87 (24.4\%) were confirmed to be suffering from JE. Among the confirmed cases, anti-JE IgM antibody was found in $49(56.3 \%) \mathrm{CSF}$ samples and $21(24.1 \%)$ serum samples. In $17(19.5 \%)$ cases both CSF \& serum samples were found to be positive. Male preponderance $(64.4 \%)$ was seen among the infected patients where majority $(61 \%)$ of patients were below 15 years of age. Infection was mostly predominant (95.4\%) during the rainy season (June-October). Among confirmed cases fever $(95.4 \%)$, altered sensorium $(51.8 \%)$, seizure (32.9\%), coma (22.9\%), headache $(25.3 \%)$ and neck rigidity $(6.9 \%)$ were the major clinical findings. Most positive cases were from the state of Meghalaya (68.9\%; mainly from East Khasi Hills \& Ri-Bhoi Districts) followed by the neighbouring states of Arunachal Pradesh $(17.2 \%)$ and Assam (10.3\%). Information generated from this study may be used for further analysis of molecular epidemiology with characterization of the circulating viral strains in various parts of the NE region. Further the study will be helpful for designing suitable control and prevention strategy in this region. 
Development of single tube multiplex Reverse Transcriptase Loop Mediated Isothermal Amplification Method for simultaneous detection of West Nile \& Japanese encephalitis virus

\section{Email ID for Correspondence: priya05120@gmail.com}

West Nile virus (WNV) is an arthropod-borne virus that belongs to the genus Flavivirus of the family Flaviviridae and is a member of the Japanese encephalitis virus (JEV) serocomplex that includes JEV. West Nile virus (WNV) and Japanese Encephalitis virus are neurotropic Flaviviruses that have emerged globally as a significant cause of viral encephalitis. Currently, there are no effective therapies or vaccines against WN and JEV infection for use in humans. Therefore, the prevention of WNV and JEV invasion is an important public health concern in regions that have close links with areas in which $\mathrm{WN}$ and JEV is endemic.The early confirmatory diagnosis of WNV and JEV infections are important for timely clinical management and epidemiologic control in areas where multiple Flaviviruses are endemic. Present study was aimed to develop (mLAMP) assay targeting env gene for detection of West Nile and Japanese Encephalitis virus. We therefore established a multiplex real- time Loop Mediated Isothermal Amplification assay to simultaneous detect and distinguish West Nile and Japanese Encephalitis RNA in single reaction. Two target sequences, one specific for West Nile and the other specific for Japanese Encephalitis virus were amplified by specific LAMP primers in the same reaction tube. After amplification at $650 \mathrm{C}$ for $60 \mathrm{~min}$ the amplified products were subjected to melting curve analysis and thus could be distinguished based on the different melting temperatures (Tm values) of the two specifically amplified products.

\section{Biocontrol of cholera using Vibrio phage Vmj1 in fresh water microcosm of North India}

\section{Naveen Chaudhary, Balvinder Mohan, Neelam Taneja}

Email ID for Correspondence: chaudharynaveen.999@gmail.com Department of Medical Microbiology ${ }^{1,2,3}$ PGIMER, Chandigarh160012, India

Biocontrol of cholera using Vibrio phage Vmj1 in fresh water microcosm of North India. Cholera remains an important public health problem in India. Cholera outbreaks frequently occur in our region in North India One-third of India's population lives under the threat of cholera. Vibrio cholerae serogroups $\mathrm{O} 1$ is the currently associated serogroups with epidemic and pandemic cholera. Cholera outbreaks frequently occur in our region in North India.In our previous work on ecology of V.cholerae in fresh water environments, we could demonstrate $11.1 \%$ of water samples from outbreak affected areas to be positive for $V$. cholerae O1.We collected 117 environmental samples including water and sewage samples from choleraaffected areas during the time of outbreaks, viable but nonculturable (VBNC)V.cholerae O1 was demonstrated in $40.69 \%$ samples from cholera-affected areas.It has been previously demonstrated that cholera stools contain lytic vibriophages and they also are important in environmental biocontrol of cholera.

Forty five samples of community sewage water and drinking water from five different cholera affected sites in North India including Manimajra, Ramdarbar, Chandigarh Ludhiana and Ambala were collected Bacteriophage isolation and purification was done by filtration and ultracentrifugation. We isolated total five vibriophages
(11.1\%) acting against $V$. cholerae O1 Ogawa serotype Out of five,4 phages were isolated from sewage (Ramdarbar,Manimajra, Ambala,Ludhiana,one phage from each site). One phage was isolated from drinking water (Ludhiana). Only one phage (VMJ1) could be propagated which was further characterised.by transmission electron microscopy and SDS -PAGE.

We studied the killing efficiency of VMJ1against V. cholerae O1 Ogawa (isolated from a recent outbreak of 2015) in Sukhna Lake fresh water microcosm.Phage VMJ1 and V. cholerae O1 were grown in continuous culture by using lab scale fermenter. Growth kinetics was studied by using spectrophotometer and readings were taken after every $5 \mathrm{~min}$ for $22 \mathrm{~h}$ automatically. A significant reduction in the Vibrio growth was seen as compared to control $(p<0.01)$ suggesting that this phage may be a good candidate for biocontrol of $V$. cholerae infection.

\section{Molecular Mapping of Enteroviruses causing HFMD from South India}

Ferdinamarie Sharmila $\mathbf{P}^{1}$, Christi $\mathbf{R}^{1}$, Rahul Dhodapkar ${ }^{1}$

Department of Microbiology, JIPMER Puducherry ${ }^{1}$

Hand, foot and mouth disease (HFMD) is a communicable disease affecting infants and children and rarely adults. It is an acute viral infection caused by a group of Enteroviruses, including Coxsackievirus A16 (CA16) and Enterovirus 71 (EV71). The disease is usually mild characterized by fever, painful sores in the mouth, and a rash with blisters on hands, feet and also buttocks. Infection with EV71 can sometimes cause mortality in children.

In recent years the incidence has increased in South East Asia including India. HFMD was first reported in India from Calicut (Kerala) in 2003, thereafter outbreaks were reported from Maharashtra, Assam, West Bengal, Odisha, Rajasthan, Jammu and Kashmir. Not much reports are available from Tamilnadu \& Pondicherry, hence this study.

Mass polio vaccination has eliminated polio viruses from the gut, thereby increasing the chances of coxsackie viral and echoviral infections. This could be one of the possible reasons for the increased incidence especially in India. A total of 132 cases were identified and included from the dermatology and Venereology clinics JIPMER. Throat swab specimens were used for RNA extraction. qRT PCR was done to confirm the presence of Enteroviruses following which conventional PCR was done for EV71, CV6, and CV16 separately targeting the VP1 region. Sequencing was undertaken to identify the presence of any mutation and to build a phylogenetic tree using MEGA. Out of the 132 samples collected 60 samples turned out to be positive for Enteroviruses by qRT PCR targeting the 5'UTR region. The conventional PCRs showed positivity for EV71-13, CV6-19, CV16-1, and co-infection of EV71 and CVA6 in 10 cases respectively. Sequence analysis revealed few variations and the strains obtained from our study was closer to South East Asian strains. 


\section{Norovirus infection and disease in a cohort study in Vellore, India}

\section{Maheswari K, Hanusha J, Nirmal Kumar, Sidhartha Giri, Gagandeep Kang}

Department \& Institution: Wellcome Trust Research Laboratory, Christian Medical College, Vellore

Norovirus are the second most cause of acute viral gastroenteritis after rotavirus infection in all age groups worldwide. Majority of the studies on norovirus associated diarrhea are hospital-based and very few are community-based studies. To compare the prevalence of norovirus infection in symptomatic and asymptomatic stool samples collected from children from birth up to the age of 3 years in Vellore. A total of 1711 diarrheal and 1400 control stool samples were tested from a birth cohort of 373 children, followed up for 3 years during 2002-2006. In the cohort study, surveillance stool samples were collected once every 2 weeks during the 3 years of follow up. For each diarrheal episode, we selected a non-diarrheal/control sample from the same child with no diarrhea in the \pm 2 weeks period. All the samples were screened for norovirus genogroups GI \& GII using real time RT-PCR (qRT-PCR) targeting ORF 1 and 2 region of the genome. The samples positive for norovirus GI and/or GII by qRT-PCR were genotyped by conventional PCR followed by Sanger sequencing. Of 1711 diarrheal episodes, $19.1 \%$ (327/1711) stool samples were positive for norovirus (56 GI, 263 GII, 8 mixed). 59.4\% (38/64) of samples positive for GI, and $58.3 \%$ (158/271) of samples positive for GII were genotyped. Of the 1400 control samples, $18.4 \%$ (258/1400) stool samples were positive for norovirus (51 GI, $197 \mathrm{GII}, 10$ mixed) by qRT-PCR. A total of $145(54.1 \%)$ qRT-PCR positive control samples were genotyped. GI.3 and GII.4 were the most common genotypes in both diarrheal and control samples. In this reports, this study re-emphasizes high rate of symptomatic as well as asymptomatic norovirus infections in the community, and found GI.3 and GII.4 as the most common strains circulating in the community.

\section{Role of Viral Hemorrhagic Fever (VHF) laboratories network in strengthening of VHF surveillance in India}

\section{Yadav PD, Sahay RR, Shete AM, Pradip Barde, Amita Jain, Sapkal GN, Bharti Malhotra, Kakru DK, P Vijayachari, Bhagirathi Dwibedi and Mourya DT}

Email ID for Correspondence: dr.rima.sahay@gmail.com ICMR-National Institute of Virology, Pune, Maharashtra, India

Viral Hemorrhagic Fever (VHF) diseases caused by RNA viruses belonging to the families; Filoviridae, Arenaviridae, Nairoviridae and Flaviviridae. Emergence and re-emergence of VHFs are major public health concern. Under Global Health Security Agenda (GHSA), ICMR-NIV, Pune is continuously working in collaboration of Centers for Disease Control and Prevention (CDC), Atlanta, USA since 2015. Multisite six VHF laboratories network were established in India to understand the presence of Kyasnur Forest Disease virus (KFDV) and Crimean Congo Hemorrhagic Fever virus (CCHFV) in the region where the presence is not known and also to screen the other VHF causing viruses like DENV, CHIKV and ZIKV. 6479 Acute Febrile Illness (AFI) cases enrolled from September 2016 to December 201. Tested for DENV, CHIKV and ZIKV. Negative samples were tested for CCHFV and KFDV. SPSS and Epi info software were used for analysis. Positivity observed for DENV (16.48\%), CHIKV $(8.52 \%)$ and dual positivity $(2.75 \%)$. No positivity for ZIKV. Negative samples were tested and found to be negative for CCHFV and KFDV, but looking at small denominator of tested samples, it will be difficult to say that no presence was detected from these centres. Diagnosis of DENV and CHIKV is mainly based on the clinical case definitions of suspected, probable \& confirmed cases given by NVBDCP. Screening of DENV \& CHIKV positive cases had increased to 789 with $63.32 \%$ sensitivity and 644 with $88.22 \%$ sensitivity respectively by new proposed case definition. Varying seasonal trends for CHIK and DEN were observed. Study provide VHF network based prevalence of DENV/CHIKV. KFDV and CCHFV screening on non-dengue, nonchikungunya cases helped in strengthening the surveillance. The capacity building of these six biomedical laboratories had helped in preparedness of country. Slight modification in standard case definition, higher sensitivity was achieved for screening of DENV and CHIKV.

\section{Clinically significant substitutions for genotype specific Direct acting antivirals}

\section{Poonam Kanta, Varun Chauhan, Mini P Singh}

Department of Virology, Post Graduate Institute of Medical Education and Research, Chandigarh, 160012

Recent development of potent direct antiviral (DAAs) for HCV have revolutionized the treatment of Hepatitis $\mathrm{C}$ with $>90 \%$ patients achieving Sustained Virological Response (SVR 12) with these newly emergent pangenomic antivirals. However, recently resistant associated substitutions (RASs) have been observed to occur which are significantly correlated with the failure of treatment. In such scenario it will be pertinent to explore the exact mechanism of interaction patterns between the available DAAs and various known mutants of NS5A protein to answer the mechanism for their development of resistance to DAAs. The aim of present study was to determine the difference in the interaction patterns of available DAAs and various combinations of RASs in NS5A protein well known for genotype 1 (1a and 1b). All the reference sequences were obtained from nucleotide database of Pubmed (https://www.ncbi.nlm.nih.gov/ pubmed/nucleotide) for HCV genotype 1 (1a and 1b).The well known hot spots which are clinically correlated with the resistance for Ledipasvir in NS5A were selected (amino acid position 28, 30, 31, 58,93 ) for genotype 1 and the reference was changed to mutant type according to the genotype specific RASs such as M28A/T/V/G, Q30/ $\mathrm{K} / \mathrm{E} / \mathrm{L} / \mathrm{H} / \mathrm{R}, \mathrm{L} 31 \mathrm{I} / \mathrm{M} / \mathrm{V}, \mathrm{H} 58 \mathrm{D}, \mathrm{Y} 93 \mathrm{~F} / \mathrm{H} / \mathrm{N} / \mathrm{C}$ for genotype 1a, L28 M, R30G/H/Q, L31F/I/M/V, P58A/L/S/D, Y93C/H/I/R for genotype $1 \mathrm{~b}$. The models were generated for the wild type and mutants using Galaxy WEB online web server. Further the models generated were refined and the quality of the models were checked by Ramachandran Plot analysis.Interacting patterns were studied with three DAAs (ledipasvir and velpatasvir) and wild and mutant proteins using molecular docking approach. The Ramachandran plot analysis revealed that the models generated were of good quality showing almost more than $90 \%$ of the amino acids in favored region. Further the molecular docking analysis revealed significant differences in the free energy of the NS5A gene on acquiring such mutations, thus conferring resistance to these drugs. Among the hotspots included in the present study, the amino acid substitutions at position 58 and 93 were identified to be most significant in terms of energy change in the $3 \mathrm{D}$ model of the protein. The study provides an in depth understanding of drug resistance mutations in the NS5A gene of the HCV genotype 1 and will further help the researchers in developing an effective strategy for $\mathrm{HCV}$ treatment. 
Comparison of rotavirus associated diarrhea in children $<5$ years of age in an Outpatient and Inpatient Setting in Christian Medical College, Vellore, India

\section{Priya Hemavathy. R, Archana Sriraman, Rajesh.A, Sidhartha Giri, Gagandeep Kang}

Department \& Institution: Wellcome Trust Research Laboratory, Christian Medical College, Vellore

Diarrhoeal diseases are a major cause of hospitalizations and child deaths globally. Of India's 78,000 rotavirus associated annual deaths of children, about 59,000 occur in the first 2 years of life. To compare the prevalence of rotavirus associated diarrhea in children $<5$ years of age in the outpatient and inpatient settings in Christian Medical College (CMC), Vellore, over a 4 years period (July 2012-June 2016), and also to evaluate the genotype diversity of rotavirus causing diarrhea in the two settings. Stool samples were collected from the enrolled children (under five age group) from inpatient and outpatient settings of CMC Vellore, after obtaining informed consent from parents/guardian. The samples were initially screened for rotavirus VP6 antigen using commercial Enzyme Immunoassay (Rotaclone) kit. For the EIA positive samples, genotyping was performed to determine the G (VP7) and P (VP4) types using conventional RTPCR. A total of 1337 children from the inpatient setting and 775 children from the outpatient setting, who had provided a stool sample, were included in the 4 years data analysis. Of the 775 children from the outpatient setting, $91(11.7 \%)$ were positive for rotavirus by EIA, compared to $29.3 \%(392 / 1337)$ rotavirus positivity in the inpatient setting. The common rotavirus genotypes in the outpatient setting were; G1P[8] (48.4\%), G2P[4] and G9P[4] (13.2\% each). In the inpatient setting, G1P[8] (62.6\%), G2P[4] (8.4\%), and G9P[4] (8.2\%) were common. Mixed genotyped were found in $5.5 \%$ and $7.9 \%$ in the outpatient and inpatient settings respectively. Partially typed and untyped samples comprised $5.5 \%$ and $3.3 \%$ in the outpatient setting, compared to only $1.1 \%$ and $1.8 \%$ in the inpatient setting respectively. Rotavirus associated diarrhea was 2.5 times more common in the inpatient settings compared to outpatient, suggesting that rotavirus associated diarrhea causes more severe episodes resulting in hospitalization.

\section{GFP tagging enables expression of human $T$ cell lymphotropic virus-1 p30 protein in bacterial system}

\section{Namdev $\mathbf{P}^{1}$ and Anupam $\mathbf{R}^{\mathbf{1}^{*}}$}

Email ID for Correspondence: priyankanamdev01@gmail.com Department of Biotechnology ${ }^{1}$, Dr. Harisingh Gour University, Sagar (M.P.), India

Human T lymphotropic virus type -1 (HTLV-1) is a complex deltaretrovirus and the only human malignant retrovirus. HTLV-1 is the causative agent of an aggressive malignancy called Adult T Cell Leukemia (ATL). pX region of the viral genome encodes an accessory protein $\mathrm{p} 30$ which is reported to be involved in promoting the viral spread, persistence and also in viral gene regulation at the transcriptional and post transcriptional levels. p30 competes with Tax for p300 binding at transcriptional level and involved in nuclear retention of tax/rex mRNA to curb the viral gene expression promoting latency. p30 modulate cellular environment to aid cellular transformation by binding various host proteins such as ATM, REG $\gamma$, PRMT5 and by altering DNA damage repair mechanisms, cell cycle regulation. However, the low expression level and instability of p30 in vivo have been major hurdles in studying its structure-function relationship in the context of HTLV-1 pathobiology. HTLV-1 p30 protein expression in large quantities was carried out in E. coli using a bacterial expression vector pRSET/EmGFP which expressed p30 by itself (Swap clone) and p30-GFP fusion protein (Insert clone). The expression was tested by immunoblotting. MALDI-TOF mass spectrometry analysis was performed to confirm the identity of p30. Expression studies of the p30 protein led us to conclusion that fusing GFP stabilizes p30 and enables its expression in bacterial system. However, the expressed p30 is going into insoluble fraction. SDS and urea were used to solubilize p30 and was purified for the first time using the His-tag affinity chromatography. The mass spectrometry analysis confirmed the identity of HTLV-1 p30. Large amounts of p30 protein will enable better insights into host protein interactions, molecular and cellular roles of p30 in HTLV-1 life cycle and pathogenesis which would be instrumental to develop novel treatments for ATL.

\section{Distribution of rotavirus genotypes in Indian children $<5$ years of age hospitalized for diarrhoea}

\section{Divya P, Priya Hemavathy R, Archana Sriraman, Sidhartha Giri, Gagandeep Kang}

Email ID for Correspondence: divyamicrocmc@gmail.com Department \& Institution: Wellcome Trust Research Laboratory, Christian Medical College, Vellore

Rotavirus causes an estimated 11.37 million episodes of acute gastroenteritis (AGE) in children $<5$ years annually in India, and approximately 78,000 deaths. The diversity of rotavirus genotypes causing diarrhoea varies across geographical regions. To compare the genotype diversity of Group A rotavirus causing diarrhoea in children $<5$ years of age in north and south India during a four year multisite hospital based surveillance study from 2012 to 2016. Stool samples were collected from children $<5$ years hospitalized with diarrhoea from seven sites across India during 2012-2016, which included 5 southern (Vellore, Trichy, Kolenchery, Hyderabad, Tirupati) and 2 northern (Delhi, Ludhiana) sites. Samples were collected after obtaining informed consent from parents/guardian. All stool samples were screened for group A rotavirus by ELISA (Rotaclone). The EIA positive samples were genotyped by reverse-transcription polymerase chain reaction. Of the 5834 samples from the 7 sites during the four years surveillance, 2069 (35.5\%) were positive for rotavirus by EIA. Genotyping was performed for 2010 (97.1\%) samples. G1P[8](56.3\%), G2P[4](9.1\%), G9P[4](7.6\%), G9P[8](4.2\%), and G12P[6](3.7\%) were the common genotypes in southern India and G1P[8](36\%), G9P[4](11.4\%), G2P[4](11.2\%), G12P[6](8.4\%), and $\mathrm{G} 3 \mathrm{P}[8](5.9 \%)$ in northern India. Mixed genotypes were more common in the north $(10.6 \%)$ than the south $(6.6 \%)$. The proportions of partially typed and untyped samples were more common in the southern sites $(1.3 \%$ and $2.5 \%$ in the south, compared to $0.5 \%$ and $1.2 \%$ in the north respectively). The study highlights the high burden of rotavirus gastroenteritis in India and the diversity of rotavirus genotypes across different geographical regions. 
Association of NKG2A Gene Polymorphism $(A>G)$ in chronic Chikungunya infection in Western Indian population

\author{
Anuradha S Tripathy, Mohini A Ganu, Sonam Lata, Sneha \\ Senapati
}

Email ID for Correspondence: sonam.bdu@gmail.com

Hepatitis Group, ICMR-NIV, Pune

Studies on humans have shown the participation of NK cells in the early control of Chikungunya, association of higher NK cell inhibitory receptors expression and lack of NK cell functionality with chronic Chikungunya infection. Hence we aimed to investigate an association between NKG2A (a NK cell inhibitory receptor) gene polymorphism $(\mathrm{A}>\mathrm{G})$ and chronic Chikungunya infection in western India. Methods: one hundred and sixty three patients with chronic Chikungunya and 163 Chikungunya negative healthy controls from western India were studied for NKG2A gene polymorphism $(A>G)$ by PCR and RFLP methods and analysis was done using SPSS software. Results: The SNP rs2734440 in NKG2A was associated with an increased susceptibility risk for chronic chikungunya.The AA genotype was more frequent in healthy controls than the patients with chronic Chikungunya $(52.14 \%$ vs. $32.51 \%$ ) with a p-value 0.003 , suggesting that subjects with showed AA genotype were protective from chronic Chikungunya indicating their resistance from progression to Chikungunya chronicity. The frequency of GG genotype was more in patients with chronic Chikungunya than the healthy controls ( $26.99 \%$ vs. $11.65 \%)$ with a p-value 0.0005 indicating the susceptibility of the genotype. Conclusion: Association of GG genotype of NKG2A gene as a susceptible gene and the emergence of AA genotype as a resistant gene towards Chikungunya virus infection is being reported for the first time. Our results suggest that genetic susceptibility and/or resistance to Chikungunya infection may be modulated by genes coding NK cell inhibitory receptors.

\section{Hepatitis E virus seroprevalence among blood donors in Pune, India}

\section{Tripathy $\mathrm{AS}^{1}{ }^{*}$, Puranik $\mathrm{S}^{2}$, Sharma $\mathbf{M}^{1}$, Chakraborty $\mathrm{S}^{\mathbf{1}}$, Dekate} U2 ${ }^{1}$

Email ID for Correspondence: anuradhastripathy@hotmail.com, sharmameenal169@gmail.com

Hepatitis Group, National Institute of Virology, Pune,Sus Road, Pashan, Pune, Maharashtra, India Department of Pathology, B.J. Medical College and Sassoon General Hospitals ${ }^{2}$, Pune, India

Blood transfusion is a recently reported route of HEV transmission. It is a bigger concern in regions where large scale HEV genotype 1 infections occur causing more severe disease. The present study aims to assess the prevalence and rate of HEV infection in the blood donors of Pune, India. A total of 2447 healthy blood donors were screened for anti-HEV IgG and IgM antibodies. Anti-HEV IgM antibody positives were further subjected to ALT measurement, HEV RNA detection, viral load quantification and phylogenetic analysis. AntiHEV seroprevalence rate was $17.70 \%$, while IgM prevalence rate was $0.20 \%$. An age dependent increase in $\mathrm{IgG}$ seropositive rate was observed. Two of 5 IgM-positives tested positive for HEV RNA. The viral load ranged from $3.5 \times 104$ to $4.6 \times 105$ copies $/ \mathrm{ml}$ and belonged to HEV genotype 1 . HEV prevalence rate of $17.70 \%$ in the blood donors of Pune, India, a developing country, goes at par with the developed countries. Current data of $0.20 \%$ [5 of 2447] blood donors positive for anti-HEV IgM and 2 of them being HEV RNA positive suggest a need for consideration of cost-effective evaluation towards pooled HEV RNA testing in blood banks. Key words: Hepatitis E virus, blood donors, age, seroprevalence, western India.

\section{Role of antigen-antibody combination ELISA in the diagnosis of Hepatitis $\mathrm{C}$ virus disease}

\section{Jayakar $\mathbf{S}^{1}$, Dhodapkar $\mathbf{R}^{2}$, Hamide $\mathbf{A}^{3}$, Parameshwaran $\mathbf{S}^{4}$}

Email ID for Correspondence: jayakar_sunil@yahoo.com Department of Microbiology ${ }^{1,2}$ Department of Medicine ${ }^{3}$ Department of Nephrology ${ }^{4}$ JIPMER, Puducherry,India

To assess the diagnostic yield of "Antigen-Antibody" combination ELISA assay versus the antibody only ELISA assay in detection of HCV among Acute Hepatitis patients and Hemodialysis patients. Currently available antibody detection assays have a long window period and detects Hepatitis $\mathrm{C}$ virus infection only when $\operatorname{IgG}$ antibodies are present.Antibody assays also have a high false reactivity.Hence the need of an improved diagnostic kit in early detection of the hepatitis $C$ virus infection. Serum samples were collected from patients with Acute Hepatitis and End Stage Renal Disease (ESRD) on Hemodialysis. The samples were subjected to "Antibody"(3rd generation ELISA) and "Antigen-Antibody" combination(4th generation ELISA) assay using commercial kits. Positivity of Hepatitis C by using combination "Antigen-Antibody"(4th gen ELISA) and only Antibody assay (3rd gen ELISA) is expressed as proportion with $95 \%$ confidence interval.Comparison of proportion of positivity between combination "Antigen-Antibody" assay and only Antibody assay was done using Chi square test among CKD and viral hepatitis patients. From the 417 samples collected, 243 belonged to CKD and 174 were acute hepatitis patients.Out of the 243 CKD patients 12 were positive by Antigen-Antibody assay and 8 were positive by Antibody assay, Acute hepatitis group, 6 were positive by "Antigen-Antibody" kit while only 1 was positive by Antibody assay. None of the samples which showed positive by Antibody assay were negative by "Antigen-Antibody" assay. Among the CKD patients comparison of both kits showed a difference of $0.017 \%$ with $95 \%$ CI of -1.5 to $1.6(p=0.92)$ while acute hepatitis patients had a difference of $5 \%$ with $95 \% \mathrm{CI}$ of $1.1-9.6 \%(p=0.011)$. The study states the higher diagnostic yield of $\mathrm{Ag}-\mathrm{Ab}$ assay among acute hepatitis patients.

\section{Hepatitis C Virus coded proteins modulate cellular metastasis suppressor $\mathrm{Nm} 23-\mathrm{H} 1$ and promote cell migration and invasion}

\section{Lohit Khera $^{1,2}$, Catherine Paul ${ }^{1}$, Rajeev Kaul ${ }^{3 *}$}

Department of Microbiology,University of Delhi South Campus ${ }^{1,3}$ New Delhi, Department of Molecular Cell Biology,Weizman Institute of Science ${ }^{2}$, Israel

Hepatitis C Virus (HCV) is the major etiological agent of Hepatocellular carcinoma (HCC) which is the third most common cause for cancer related deaths. HCV induced HCC is a multi-step process which involves alteration of several of host regulatory pathways. One of the key features of HCV associated hepatocellular carcinoma is the metastasis of cancer cells to distant organs. Human Nm23-H1 is one of the best studied metastasis suppressor protein which has been shown to be modulated in many human cancers. Our work shows that $\mathrm{HCV}$ envelope protein E1 protein expression as well as $\mathrm{HCV}$ 
infection induces pro-metastatic effect on cancer cells which is simultaneous to Nm23-H1 transcriptional down-regulation and Nm23-H1 protein degradation. Moreover, Nm23-H1 intracellular localization is significantly altered in cells expressing HCV E1 protein. Importantly, overexpression of $\mathrm{Nm} 23-\mathrm{H} 1$ can rescue the cancer cells from pro-metastatic effects of HCV E1 and HCV infection. We further show that $\mathrm{HCV}$ capsid protein 'Core' can co-localize and interact directly with $\mathrm{Nm} 23-\mathrm{H} 1$ within the cancer cells. This interaction results in modulation of anti-metastasis properties of Nm23$\mathrm{H} 1$. HCV core promotes $\mathrm{Nm} 23-\mathrm{H} 1$ protein sumoylation, degradation, as well as transcriptional downregulation. Our study now provides evidence for role of $\mathrm{HCV}$-Core and E1 proteins as pro-metastatic proteins which can modulate functions of cellular metastasis suppressor Nm23-H1 in HCV mediated cancer metastasis.

\section{Circulation of two Mumps virus (MuV) Genotypes in Assam, India during 2016 and 2017}

\section{Sarmah K, Borkakoty B, Sarma K, Bora PK}

Email ID for Correspondence: kimmi.sarmah@gmail.com Regional Medical Research Centre, N.E. Region, Dibrugarh, Assam, India. Pin- 786001

Mumps is a vaccine preventable disease and therefore Mumps vaccine has been used as a component of the trivalent Mumps-measlesrubella vaccine. Recent reports suggesting the re-emergence of Mumps infection worldwide in the vaccinated populations signifies the declining efficacy of the vaccine. Waning of immunity attributed to the variations between the circulating and the vaccine strains remains one of the major cause of Mumps outbreaks. Moreover, Mumps is not recognized as a public health problem in India due to lack of comprehensive reports on its infection, documentation of clinical cases and published studies though it causes high degree of morbidity and severe complications in young adults. The present study was aimed to characterize the circulating $\mathrm{MuV}$ strains in Dibrugarh district of Assam, India during 2016 and 2017. A total of 66 (throat swab/blood) samples were collected from patients who presented with fever and unilateral/bilateral parotitis. Blood samples $(n=23)$ were investigated for IgM antibody against Mumps and molecular detection was carried out in throat swab samples $(n=66)$ utilizing TaqMan assay. Genotyping through sequencing was done targeting the small hydrophobic ( $\mathrm{SH})$ gene and its genetic variations and phylogenetic analysis was performed. IgM antibody was detected in 57\% (13/23) cases and MuV RNA was detected in 55\% (36/66) cases, of which $58 \%$ were male and $42 \%$ were female. Majority of the cases were children. Sequence and phylogenetic analysis of the $\mathrm{SH}$ gene revealed simultaneous circulation of Genotype C (76\%) and G (24\%) in the corresponding years. To our knowledge this is the first report of circulating MuV strains from North-east India. The study provides important genetic baseline data for the development of prevention and control measures of mumps. Further bioinformatics analysis which is in process would shed more information on the genetic diversity of $\mathrm{MuV}$ and its evolution.
In silico mapping of CD81-E2 interaction across $\mathrm{HCV}$ genotypes 1 and 3

\section{Bhattacharjee $\mathbf{C}^{1}$, Nandy $\mathbf{S}^{2}$, Das $\mathbf{P}^{1}$ and Mukhopadhyay Aa*}

Department of Life Sciences, Presidency University ${ }^{1}$ Kolkata, India; Department of Biochemistry \& Biophysics, University of Kalyani ${ }^{2}$, Kalyani, India

Hepatitis C Virus is a blood borne pathogen responsible for chronic hepatitis in more than 71 million people worldwide. Wide variation across strains and genotypes are one of the major hurdles in therapeutic development and treatment. While genotype 1 remains the most extensively studied and abundant strain, genotype 3 is second most prevalent and more virulent. We have compared differences in the glycoprotein E2 across HCV genotypes at nucleotide, protein and structural levels using various tools of bioinformatics. E2 sequences from 29 strains across genotypes $1 \mathrm{a}, 1 \mathrm{~b}, 3 \mathrm{a}$ and $3 \mathrm{~b}$ revealed a preference for C-richness which was attributed to a distinct bias for C-rich codons in genotype 1 and to a lesser extent in genotype 3. Amino acid level comparison revealed an $\mathrm{N}$-terminal region conspicuously conserved with majority of the changes at the C-terminal half of the proteins. In silico models of E2 glycoproteins and docking analysis with the energy minimised PDB-CD81 model revealed unique interacting residues in both E2 and CD81. E2 of genotype 3a and CD81 had the strongest interaction. In conclusion this is the first comprehensive study comparing E2 sequences across genotypes 1a, $1 \mathrm{~b}, 3 \mathrm{a}$ and $3 \mathrm{~b}$ revealing stark genotype-specific differences which requires more extensive investigation. Elucidating amino acid differences among dengue sero
and genotypes and their implication in dengue virulence

Email ID for Correspondence: bibhu.talk@gmail.com Bibhudutta Mishra, Dr Raviprasad Aduri,BITS Pilani K K Birla Goa campus. Goa, India

Dengue virus (DENV) is one of the most important mosquito-borne virus causing dengue fever (DF) and severe dengue (SD). It exists in four different serotypes, DENV-1, 2, 3, and 4. Again these serotypes are classified into different genotypes based on the E-NS1 gene sequence. One of the factors attributed to the severity of disease is the intrinsic genetic composition of the virus. In this study we focused on how the differences in the amino acid sequence of structural and nonstructural proteins may lead to varying degrees of pathogenesis. Complete genomes of Dengue from NCBI are classified into serotypes/genotypes using Vipr database. A consensus sequence for each of the proteins is generated using Clustal Omega. Amino acid variation within a given protein among the genotypes of a serotype is generated using in-house python scripts. The observed amino acid variation is then mapped into the experimentally determined structure of the proteins, wherever available or onto the homology model of the protein. Both the structural and non-structural proteins show high amino acid sequence conservation within a given serotype, however there is a marked variation between the serotypes (for example, the linker region of NS3). Most of the amino acid variation observed is mainly conservative in nature (i.e. polar to polar). Nevertheless, we observed the following changes more frequently than the others: Aromatic (Y or F) - L; (2) D/E - N/Q; (3) N/Q - H; (4) A/S/G - T; (5) $\mathrm{Y} / \mathrm{F}-\mathrm{H}$. Mapping these differences onto the structures revealed that most of these changes are in the loop regions that may play an important role in protein-RNA interactions. 
The observed amino acid variation in the dynamic loop regions of $\mathrm{E}$ and NS3 protein suggest possible role of these changes.

\section{Importance of Rab1a in HCV endocytosis}

\author{
Bhattacharjee $\mathrm{C}^{\mathbf{1}}$, Adhikary $\mathrm{S}^{\mathbf{1}}$, Mukhoapdhyay $\mathrm{A}^{\mathbf{1}}$ \\ Email ID for Correspondence: aparna.dbs@presiuniv.ac.in \\ Molecular Virology Laboratory ${ }^{1}$, Department of Life Sciences, \\ Presidency University, Kolkata, India
}

Hepatitis $\mathrm{C}$ virus (HCV) is an enveloped RNA virus, belonging to the family Flaviviridiae. It is the leading cause for chronic liver disease and hepatocellular carcinoma. Viral entry is via different hepatocellular receptors, especially tetraspanin CD81. Interaction between CD81 and viral glycoprotein E1E2 is followed by clathrin mediated endocytosis in a time dependent manner. In our present work using GFP labelled HCV pseudoparticles (HCVpp), we are investigating the time line of HCV entry into hepatocytes. Receptor mediated endocytosis via clathrin coated vesicles has been previously shown to be regulated by the small GTPases, Rabla, particularly at the early endocytic sorting step. In this study, we investigated the role of Rabla in HCV endocytosis using a Rabla knocked down cell line. In a timedependent study, GFP and luciferase tagged HCVpp trafficking was assayed in Rab 1a knocked down cell line and compared to the parental cell line, Huh7. Entry events were studied via confocal microscope and western blot. Our results show the importance of Rabla in HCV trafficking.

\section{Studies of antiviral molecules targeting Chikungunya virus specific serine protease}

\section{Fatma B, Saini R, Sharma R, Kesari P and Tomar S}

Department of Biotechnology, Indian Institute of Technolgy, Roorkee, India

Chikungunya, an Alphavirus (genus of Togaviridae family) is enveloped, arthropod borne, positive-sense single-stranded RNA virus. Re-emergence of the Chikungunya occurs time and again across the world but till date, no therapeutic is available to combat the Chikungunya virus. The capsid protein of Chikungunya is present at the N-terminus of the structural polyprotein. It consists of two domains: the amino terminus and carboxyl terminus domain. The amino terminus domain interacts with the viral genomic RNA and other capsid molecules which lead to viral genome RNA encapsidation and nucleocapsid assembly. The carboxyl terminus domain possesses auto- proteolytic activity and is also involved in virus budding. Since capsid plays a prominent role in the viral life cycle so it can be a potential target for antiviral drug development. In this study the capsid protein of Chikungunya virus has been cloned, expressed and purified using various chromatography techniques. The three dimensional structure of Chikungunya capsid protein was used for screening and identification of inhibitors from a small molecule library. The validation of screened compounds was done by using AutoDock tools 1.5.6. The identified compounds were tested for inhibitory activity against capsid protease using FRET based assay and inhibition kinetics has been done. The biochemical validated compounds have been further used to study the antiviral efficacy against Chikungunya virus in cell based culture. Our biophysical, biochemical and antiviral characterization of potential inhibitors of Chikungunya capsid will pave the path for the drug development against the Chikungunya disease.
Detection of Dengue parameters and their correlation with platelet count among patients suspected with platelet count among patients suspected with dengue infection

Neelofar Malik ${ }^{1}$ Singh S. $^{2}$, Farooq U. $^{3}$

Email ID for Correspondence: neelofarmalik537@gmail.com Department of Microbiology Teerthanker Mahaveer Medical College \& Research Centre, Moradabad

Dengue infection, an acute febrile arboviral disease has become a major public health concern. Annually, it affects up millions of people worldwide. An early and accurate diagnosis of dengue in acute phase of illness is important for identifying an epidemic and for initiation of therapy. Detection of the secreted NS1 protein is a new approach that aid in early diagnosis. Platelet count is the only non -dengue parameter that can support the diagnosis of the dengue shock syndrome and dengue. This study was done to detect dengue parameters and correlate them with the platelet count. The study was conducted in virology section of microbiology department at Teerthankar Mahaveer Medical college and research center, Moradabad (U.P.) Serum samples were collected from clinically suspected dengue cases and tested for NS1 Antigen,IgM and IgG antibody by using immunochromatographic method. The platelet count was also recorded in all samples. Out of 100 sample tested total 52(52\%) specimen were positive for one or more dengue parameters and 48(48\%) specimen were negative. Among 39(75\%) specimen were positve only for NS1 antigen and $13(25 \%)$ were positive for IgM only. Control measures along with rapid diagnosis is the key to effective management of dengue infection. Ahigh percentage of NS1 antigen in the study population indicated new infection warranting an immediate need of control of mosquitos and preventive measures.

\section{KSHV, a journey to the oncogenesis}

\section{Tathagata choudhuri}

Email ID for Correspondence: tathagata.choudhuri@visvabharati.ac.in

Departmet of Biotechnology, Visva Bharati, Santiniketan, West Bengal, India

Primary effusion lymphoma (PEL) is a rare form of B cell lymphoma with lymphomatous effusions in body cavities. HIV infected patients and patients with immune compression are mainly subjected to be infected by KSHV induced PEL. Despite of different available anticancerous remedies targeting the cellular and viral mechanisms didn't give any promising feedback with poor survival. In our recent study, we have investigated the efficacy of different component on KSHV induced PEL. The anti-cancerous property of Everolimus, 1, 25(OH)2 D3 and Theaflavin has been tested against KSHV induced PEL cell line in a dose depended manner. Cell death analysis was done by flow cytometry and protein expressions were studied by real-time PCR and western blotting. Everolimus has shown to downregulate the KSHV latent antigen expression with concurrent blocking of lytic reactivation for active virus replication and also inhibited latent antigen mediated constitutively active STAT- 3 and NF- $\kappa$ B signaling. From the co culturing experiment with immature dendritic cells, we have found the activation of dendritic cells with increase in surface expression of CD86 and HLA-DR. 1, 25(OH)2 D3 induces both death of HSHV infected PEL cells and KSHV replication in p38 and VDR dependent manner. With the dose dependent treatment of Theaflavin, the functional status of viral proteins is emphasized and apoptosis of 
normal cells is decreased. The present study with the anti-viral as well as anti-cancerous effect of those component was a step forward analysis of the KSHV associated PEL and have demonstrated a path of viral antigen disruption, viral reactivation and apoptotic and therapeutic role in the virus associated cancer. This work helps us better understanding with the host and viral molecular interactions and promising target area of further research.

\section{Seroprevalence of hepatitis B, hepatitis C and HIV infection in tertiary care teaching hospital in (UP)}

\author{
Sakshi Vishnoi ${ }^{1}$, Dr.Sudhir Singh ${ }^{2}$, Dr.Umar Farooq ${ }^{3}$
}

Email ID for Correspondence: anugoyal00023@gmail.com Department of Microbiology $y^{1,2,3}$ Teerthanker Mahaveer Medical College \& Research Centre, Moradabad, U.P.

HIV, Hepatitis B and Hepatitis C are serious global health problem. The HIV/AIDS epidemics are one of the largest public health problem of 21st century. Hepatitis B and Hepatitis C infection are associated with chronicity leading to cirrhosis and further progress to Hepatocellular carcinoma. The risk behaviors and routes of transmission of these infections are identical and hence it is observed that many patients are co-infected with one or two of these infections (HIV, Hepatitis B and Hepatitis C). Early diagnosis and appropriate treatment prevent the transmission as well as progression of the disease, further increasing life expectancy. This study was aimed to assess the prevalence of HIV, Hepatitis B and Hepatitis C infection in ANC women attending ANC clinic of tertiary care teaching hospital in western U.P.

The study was conducted in serology section of microbiology department at Teerthanker Mahaveer Hospital \& Research center Moradabad (U.P.). The serum samples received from the ANC clinic were included in the study. A total 250 samples were tested from ANC cases for HBsAg, HCV and HIV. Out of total samples, $42(16.8 \%)$ were reactive. In which $31(12.4 \%)$ were reactive for $\mathrm{HCV}$, 9(3.6\%) were reactive for HBsAg and $2(0.8 \%)$ samples were reactive for HIV. Prevalence of HIV, HBsAg and HCV among ANC cases represents their prevalence among normal population. Such studies are needed to know the prevalence of such diseases in our society which can help us to form the guidelines for patient management.

\section{Effect of dicotyledonous plant extracts on Hepatitis C virus entry events}

\section{Avik Bardhan, Aparna Mukhopadhyay, Chayan Bhattacharjee}

Email ID for Correspondence: avikbardhan1994@gmail.com Presidency University, Kolkata

Hepatitis C virus (HCV), a positive stranded RNA virus of the Flaviviridae family is the causative agent of Hepatitis which is a major health concern. The modus operandi of the virus leading to its pathogenesis is not fully understood and as such no pan genomic potent vaccines have been discovered. Although emphasis has been given on herbal medicines, most of them are polymerase inhibitors affecting the virus at the replication stage in the host cell.In India, the major problem lays in the fact that blood transfusion events lead to the transmission of $\mathrm{HCV}$ in several cases calling for a remedial strategy to block the virus at the entry step.Some of the common plants in Kolkata were selected for this study that has one of its family members conditions with established literature of its effectiveness in blocking HCV at the entry step. Also, literature survey showed the presence of certain organic active compounds that was responsible for the entry inhibition. Previously in the lab, the E2 glycoprotein was modeled de novo since no crystallization structure was available in any database. The binding interactions of the active compounds of the selected plants were checked in silico with the de novo modeled E2 glycoprotein using molecular docking tools. Also, GFP and luciferase tagged $\mathrm{HCV}$ pseudoparticles (HCVpp) were prepared in the lab to perform the entry assays and study the effects of the selected plant extracts on the HCVpp. Bioinformatics tools were employed to estimate the amino acid residues responsible for the binding interactions and hence develop an idea about the binding site thermodynamics of the E2 glycoprotein with the active compounds that lead to entry inhibition.

\section{Co-infection of Dengue with Chikungunya and Typhoid in a tertiary care center of North India}

\section{Galhotra S, Grover P, Jindal N, Dhuria N}

Email ID for Correspondence: shipragalhotra@gmail.com Department of Microbiology GGS Medical College and Hospital Faridkot

Acute febrile illness is very common in patients especially between the month of June and September. This is a common clinical syndrome of dengue, typhoid, Japanese encephalitis, Chikungunya, Leptospirosis, influenza A and Malaria. Nowadays, Co-infection with two or more infectious agents is becoming a major public health problem. A total of 1145 samples tested for dengue NS1 from May 2017 to Nov 2017 were analyzed for the presence of other febrile illness i.e. typhoid and chikungunya fever. Out of these, 260 samples were positive for NS1 Ag and these 260 samples were studied for typhoid and chikungunya co-infection by TYPHIDOT Rapid IgM Immunochromatographic test and IgM Capture ELISA respectively. Dengue NS1 Ag positivity was $22.7 \%$ (260/1145). Of these positive (260) samples, $11(4.2 \%$ ) were positive with Salmonella typhi by rapid IgM test and 9 (3.4\%) were positive for IgM antibody for Chikungunya. There was no patient which was positive for all the three infections. In our Malwa Region of Punjab dengue and typhoid coinfection exists, early diagnosis of typhoid infections in patient suffering from dengue fever is required as early antibiotic therapy in the former leads to a favorable outcome, while dengue as such has no specific treatment and is treated symptomatically. Repeated outbreaks of dengue, chikungunya/dengue co-infections are occurring in our region. So, in clinically suspected cases of dengue or chikungunya fever, it is advisable to test for both viruses in this area.

\section{Epidemiology of viral respiratory infection in Odisha, India}

Jyotsnamayee Sabat $^{1}$, Subhra Subhadra ${ }^{1}$, Bhagirathi Dwibedi ${ }^{2}$, Subrata K Palo ${ }^{1}$ Sanghamitra Pati $^{1}$

VRDL, ICMR- Regional Medical Research Centre, Bhubaneswar, Odisha $^{1,2,3,4,5}$

Acute respiratory tract infections (ARI) are an important cause of morbidity and mortality worldwide. Global burden of ARI has been estimated to be 94037000 DALYs and 3.9 million deaths (WHO 2002). The aetiology of viral ARI can be determined in $80-95 \%$ of cases. This study reports the viral etiology of respiratory illness in 
eastern India during 2010-2017. Throat/nasal swabs from 3302 hospitalised symptomatic individuals were collected from 40 different hospitals of Odisha. Nucleic acid was extracted by column separation method and subjected to RealTime PCR for qualitative detection of suspected viruses. It was observed that $20.8 \%$ cases were positive for pandemic H1N1 2009. Other viruses detected were Flu A in $12 \%$ cases followed by RSV (5.7\%), Adeno (4.2\%), Rhino (3.9\%) and Parainfluenza (3.9\%). Among all cases, $25 \%$ were children below 5 yrs of age and viral etiology was found in $34 \%$ cases. In these children, RSV was detected in $9.6 \%$ followed by Adeno in 5\% of cases. Among adults, the common viruses detected were Pandemic H1N1 2009 in $16.43 \%$ followed by Flu A in $15.3 \%$. RSV was the most common virus detected among children. Viruses like Pandemic H1N1 2009, Flu A, RSV, Adeno and PIV has caused high morbidity in the state. Also Corona, Human Boca Virus, HMPV etc. were also detected for the first time. Hence, a routine surveillance is essential to monitor the seasonality and true prevalence of these viruses to support vaccination advocacy in future.

\section{Herpes simplex virus type 2 and cytomegalovirus perigenital ulcer in an HIV infected woman}

\author{
Rawre $\mathbf{J}^{1}$, Namdeo $\mathrm{D}^{2}$, Rosaleen Das ${ }^{3}$, Khanna $\mathbf{N}^{4}$, Dar $\mathbf{L}^{5}$, \\ Dhawan $\mathbf{B}^{5}$
}

Email ID for Correspondence: jyotirawre@gmail.com Department of Microbiology, AIIMS,New Delhi,Professor, Department of Dermatology and Venereology ${ }^{4}$, AIIMS,New Delhi, Professor,Department of Microbiology ${ }^{5}$, AIIMS,New Delhi; Department of Microbiology ${ }^{5}$, AIIMS New Delhi

Genital herpes $(\mathrm{GH})$ is the most common cause of genital ulcer disease (GUD) worldwide; its association with human immunodeficiency virus (HIV) is well established. Cytomegalovirus (CMV) is an important opportunistic agent in immunosuppressed states particularly in HIV infected, manifesting as ocular and visceral involvement. Though cutaneous manifestations are rare, there are growing number of reports of concurrent Herpes Simplex Virus (HSV) and CMV infections in genital and perigenital ulcers of immunocompromised individuals. Here, we report a case of mucocutaneous HSV-2 and CMV infection in a 39-year old woman with acquired immunodeficiency syndrome, who presented with a perigenital ulcer. Scrapings from the perigenital ulcer were collected in Viral Transport Medium (VTM) for HSV culture and polymerase chain reaction (PCR) for HSV-2, CMV, Haemophilus ducreyi and Treponema pallidum. Serum samples were collected for HSV and syphilis serology. Scrapings from the perigenital ulcer were positive by PCR for HSV-2, CMV and T. pallidum, but tested negative for $H$. ducreyi. Culture and serology for HSV 2 was positive. Both VDRL and TPHA for syphilis were positive. This case underscores the importance of maintaining a broad differential diagnosis for genital ulcers in immunocompromised patients. Any genital or perigenital ulcer should be investigated to rule out CMV in an immunocompromised patient as its recognition could reflect systemic involvement and significantly affect patient care. Key words: Herpes Simplex Virus, Cytomegalovirus, Human Immunodeficiency Virus, Haemophilus ducreyi, Treponema pallidum.
Identification of mutations in Haemagglutinin gene of Influenza A H1N1 pdm09 strain from 2016-2017 outbreak in India

\author{
Akshatha $R^{1}$, Dhodapkar $R^{2}$, Ferdinamarie Sharmila $P^{3}$ Sistla $S^{4}$ \\ Wyawahare $\mathbf{M}^{5}$
}

Email ID for Correspondence: akshukusum@gmail.com Department of Microbiology $1,2,3,4$ JIPMER, Department of Medicine ${ }^{5,}$ JIPMER, India

Circulating influenza viruses are estimated by the WHO (WHO) to infect $5-10 \%$ of the population every year. Studies have shown that epidemics are most commonly caused by Influenza A (H1N1) pdm09 $(66 \%), \mathrm{B} /$ Victoria lineage $(24 \%)$ and A (H3N2) (10\%). Over the years certain mutations in amino acids at position 222 in HA gene has been associated with higher virulence and severe disease by increasing the affinity to respiratory tract mucosa. This study, the first of its kind, was done to find whether there were any similar or different mutations in the outbreak of 2016-17. The current work was carried on archived Influenza A H1N1 positive samples (qRT method) from Regional Influenza Laboratory, JIPMER. RNA was extracted using commercially available extraction kit (Roche High pure viral nucleus acid kit) according to manufacturer's instruction. Conventional one step RTPCR was done using AgPath -ID kit and pre-published primers sequences targeting the HA region. Positive PCR products were purified by ExoSAP-IT (GE Healthcare), and then subjected to Sanger sequencing using M13 primer with Big Dye Terminator Reaction Mix. Sequencing results were analysed using the MEGA 7.0 Software. Hundred samples were retrieved and were classified into 63 ILI and 37 SARI cases respectively. Only 48 samples showed positive by conventional PCR with a product size $890-976 \mathrm{~KB}$, a possible reason being that most of the samples had a high $\mathrm{Ct}$ value $(>33)$ and/or possibility of degradation in RNA. By Sanger sequencing few variations were identified and phylogenetic analysis revealed strains close to South Asian population.

\section{Antiviral drug identification by targeting nsP3-G3BP complex of Alphavirus}

\author{
Mahajan $\mathbf{S}^{1}$, Kumar $\mathbf{R}^{1}$, McInerney $\mathbf{G}^{\mathbf{M}^{2}}$, Tomar $\mathbf{S}^{1 ; *}$ \\ Email ID for Correspondence: shailfbt@iitr.ac.in \\ Molecular Virology Laboratory, Department of Biotechnology, \\ Indian Institute of Technology Roorkee, Roorkee, Uttarakhand, ${ }^{1}$ \\ India; Department of Microbiology, Tumor and Cell Biology, \\ Karolinska Institutet, Stockholm, Sweden ${ }^{2}$
}

Alphaviruses are enveloped, $70 \mathrm{~nm}$ diameter, spherical and $40 \mathrm{~nm}$ isometric nucleocapsid particles. Alphavirus infection in humans is transmitted through mosquitoes. Major symptoms of infection in humans include fever, rash, arthritis, encephalitis. The diverse interactions of viruses with the host cell and interaction of viral proteins with host proteins effectively manipulate host cell antiviral response and cellular processes. The host cells have also evolved antiviral defense mechanisms which target viral RNAs and proteins. One such mechanism of defense is stress granule formation which is a dynamic assembly of proteins regulating mRNA translation during time of stress and reduces bulk protein synthesis. Mechanism of stress granule (SGs) formation is not yet completely established but largely dependent on TIA1/R and G3BP(Ras-GAP SH3 domain-binding) protein. Role of G3BP in cells exposed to environmental stress and viral infections is well elucidated. Non-structural proteins of alphaviruses act as host defence modulator that functions by disrupting 
stress granules formed due to cellular stress response to viral infections. This disruption mechanism is explained through nsP3-mediated recruitment of human G3BP1 protein via two tandem FGDF motifs. Thus, FGDF-motif of nsP3 is directly affecting SG formation and going around the host cell defenses. So we hypothesize that by targeting G3BP and designing pharmacophores that block G3BP interaction with the viral partner will lead to novel antivirals. Molecular docking has been carried out using CDOCKER and AutoDock to predict the binding-conformation of screened peptidemimetics to the G3BP. Human G3BP has been expressed using bacterial expression system, and purified using Ni-NTA column affinity chromatography. His-Tag has been successfully removed using Tev protease followed by reverse Ni-NTA affinity chromatography. Purified protein has been crystallised and cocrystallisation with inhibitors is in progress. Antiviral studies of identified G3BP inhibitors show promising results.

\section{Expression levels of toll like receptors in the culture supernatant of microglial and neuroblastoma}

\author{
Shukla $\mathbf{M}^{1}$, Chaturvedi $\mathbf{M}^{2}$, Dhole $\mathbf{T N}^{3}$ \\ Email ID for Correspondence: tndhole@gmail.com, \\ muktishukla@gmail.com \\ Department of Microbiology ${ }^{1,3}$ Sanjay Gandhi Post Graduate Institute \\ of Medical Sciences, Lucknow U.P,Amity Institute of \\ Biotechnology ${ }^{2}$, Amity Institute of Biotechnology, India
}

Japanese encephalitis virus (JEV) is the leading cause of viral encephalitis in Asia. There is no effective method to cure this deadly disease. Activation of various pattern recognition receptors provides a first line of defense by inducing IFN- $\alpha / \beta$, proinflammatory cytokines and chemokines. These early responses are protective by activating and amplifying antiviral mediators, as well as recruiting leukocytes expressing antiviral function. However, the same mediators promote tissue damage if not dampened in a timely manner. We aimed to study the mRNA gene expression of TLRs $2,4 \& 9$ in microglial cell line BV-2 \& neuroblastoma cell line Neuro-2A by Quantitative Real time PCR (qRT-PCR). qRT-PCR was performed for total cellular RNA extracted to measure mRNA expression for TLRs $2,4 \& 9$ in the cell supernatant of BV-2 and Neuro-2A infected with JEV. Infection and treatment schedule of BV-2 \& Neuro-2A cells: The BV-2 \& Neuro2A cells were plated in 6-well plates and cultured until $70-80 \%$ confluence monolayers were obtained. After 18 to $24 \mathrm{~h}$ incubation in $10 \%$ DMEM, the cells were adsorb with JEV (108 pfu/mL) at an MOI of 5 for $1.5 \mathrm{~h}$. After adsorption, the unbound viruses were removed by washing with sterile $1 \mathrm{X}$ PBS. The cells were then incubated and mRNA for TLRs $2,4 \& 9$ was carried out at $0,3,6,12 \& 24 \mathrm{~h}$ respectively. Mock treated control cells received only 1xPBS Statistical analysis was done using graph pad prism version 5. $p$ value $\leq 0.05$ was considered significant. We are analyzing the data. The results will be discussed at the time of presentation. This study will help to identify new targets for the therapeutic treatment of JEV infection and may lead to the development of potential pharmacological targets.
RNA dependent RNA polymerase as an antiviral drug target for emerging alphaviruses

\section{Pareek A, Mudgal R and Tomar S}

Department of Biotechnology, Indian Institute of Technolgy, Roorkee, India

Alphaviruses are arthropod borne viruses which belongs to Togaviridae family. These viruses have emerged as a serious threat to human lives from past few years. Chikungunya virus (CHIKV), Sindbis virus (SINV), Venezuelan Equine Encephalitis virus (VEEV) etc. are well known members of this family. There is no medication and vaccine to cure the disease caused by alphaviruses. Therefore, development of antiviral agents specifically targeting the key viral enzymes is necessary to control the viral diseases. Alphaviruses contain a genome of $\sim 11.5 \mathrm{~kb}$ size in which the two-third part translates into the non-structural polyprotein and the other one-third part translates into the structural polyprotein. The non-structural polyprotein is processed by viral protease to form four mature replication proteins that form the replication complex in infected cells. The mature non-structural proteins formed are nsP1-4 in which nsP4 functions as a RNA dependent RNA polymerase (RdRp). nsP4, the viral RdRp uses positive-sense RNA genome and replicates its. In this study, RdRp genes of two different alpahviruses and number of their N-terminal truncation constructs have been made for expression in bacterial expression system. Sindbis virus (SINV) RdRp has been successfully purified using Ni-NTA affinity chromatography and size exclusion chromatography using AKTA purifier (GE healthcare). Preliminary studies indicate that alphavirus nsP4 in presence of nucleic acid forms higher oligomeric states. Additionally, biophysical characterization of purified protein by $\mathrm{CD}$ shows metal binding and nucleotide binding. Crystallization of protein for atomic structure determination is in progress.

\section{In silico analysis to understand more prolonged viremia in Dengue Virus Serotype-2}

Email ID for Correspondence: sudheer.bioinfo@gmail.com All India Institute of Medical Science, Bhopal

Delayed viral clearance (viremia beyond 5 days) was found significantly higher among Dengue virus-2 (DENV-2) infected cases during molecular characterization of DENV serotypes that circulated in Madhya Pradesh region during the 2016 outbreak (Unpublished data). In view of this, we performed an in silico analysis to investigate antigenic variability in DENV serotypes which might be contributing to higher ability for immune evasion and prolonged viremia. Genomewide association was carried out with existing DENV sequences from ViPR database. Immune evasion potential of infecting serotypes was ascertained by computing antigenic variability in B cell and Cytotoxic T cell (CTL) epitopes of all DENV proteins, using BepiPred software and IEDB analysis Resource, respectively. We first computed the variability-coefficient for each position in DENV polyprotein of all four serotypes. We observed that the average variability in DENV-2 polyprotein was higher as compared to other serotypes $(p<0.05)$. We also observed that NS2a protein of DENV-2 displayed highest average variability among all the proteins. Next, we computed the frequency of variability in the sequences of B cell epitopes and HLA-I binders which might be responsible for the evasion of humoral-immunity and cytotoxic T-cell responses respectively. The number of variable B-cell epitopes were significantly different between 
serotypes and highest in DENV-2 $(p<0.001)$ for variability scores $>4$. Similarly, the total number of variable HLA-I binders were significantly different $(p<0.05)$ between the four serotypes and maximum in case of DENV-2 at variability thresholds $>4$. This study reports the occurrence of maximum variability in $\mathrm{B}$ cell and $\mathrm{T}$ cell epitopes of DENV-2 among all serotypes, which is consistent with the more prolonged viremia observed in infection with this serotype.

\section{Molecular and phylogenetic analysis of Chikungunya virus outbreaks in Central India during 2016, 2017}

Email ID for Correspondence: ankita.rvl@aiimsbhopal.edu.in Regional Virology Laboratory, All India Institute of Medical Sciences Bhopal, Saket Nagar, Bhopal-462020, India

Chikungunya is now endemic in most parts of India. Central India witnessed Chikungunya virus (CHIKV) outbreaks in 2016 and 2017 with huge number of patients visiting our tertiary care hospital. The present report is a hospital based cross-sectional study in which serological and molecular investigations of patients suspected with CHIKV infection were undertaken. Further, mutational and phylogenetic analysis was conducted to study the genetic relatedness of the Bhopal strains with other Indian strains and worldwide strains. During 2016 and 2017 outbreaks, samples from Chikungunya suspected patients were collected and tested for IgM and Anti-NS1 antibody by ELISA and viral RNA was detected by RT-PCR. Some of the RTPCR positive samples were sequenced for partial E1 gene and analyzed to identify the newly emerging mutations and understand the phylogenetic relationship among 2016 and 2017 strains as well as with other Indian and worldwide strains. Phylogenetic analysis revealed the present strains to be of ECSA genotype. Emergence of a variant strain was observed in the year 2016, which became the predominant strain in this region in 2017. The strains showed high similarity with recent New Delhi strains of 2015 and 2016. The epidemic mutation A226 V emerged in 2006 outbreak was found to be absent in the current strains. Among the important mutations viz. K211E, M289 V, D284E, I317 V \& V322A observed in the recent strains, I317 V is found to emerge very recently as it is present only in Bhopal $(2016,2017)$ and New Delhi strains $(2015,2016)$. This study has identified unique mutation $\mathrm{I} 317 \mathrm{~V}$ in the $\mathrm{E} 1$ gene, which is present only in New Delhi strains till date. This study warrants continuous surveillance for mutations having epidemic potential thus aiding in prediction of future outbreak

\section{Epidemiology and genetic diversity of Sapoviruses in cases of acute gastroenteritis in children $<5$ years, 2012-2018}

\section{Blossom Benny ${ }^{1}$, Soumya Roobini ${ }^{1}$, B.Manohar ${ }^{2}$, Samarasimha Reddy $^{1}$, Sidhartha Giri ${ }^{1}$ Gagandeep Kang ${ }^{1}$, Benjamin Lopman ${ }^{3}$, Ira Praharaj ${ }^{1}$}

Wellcome Trust Research Laboratory, Division of Gastrointestinal Sciences ${ }^{1}$, Christian Medical College, Vellore, Tamil Nadu,Sri Venkateswara Medical College, Thirupathi ${ }^{2}$,Rollins School of Public Health, Emory University ${ }^{3}$, USA

Diarrheal diseases are a major cause of childhood mortality and morbidity in Indian settings, and viruses have been found to be major etiologic agents in children $<5$ years. In Indian settings, the etiologic role of sapoviruses in acute gastroenteritis and their molecular epidemiology is not well described. To study the trends in sapovirus prevalence and their genetic diversity in cases of acute gastroenteritis among hospitalized children $\leq 5$ years in a tertiary care facility in south India over a period 2012-2018. Stool samples from children $\leq 5$ years hospitalized for acute gastroenteritis at a tertiary care centre in Thirupathi, south India, collected as part of a surveillance for rotavirus disease from 2012 till February 2018 were included in the testing for sapovirus. Following viral RNA extraction, qPCR was performed targeting the ORF1 region. All samples with positive amplification with the qPCR assay, with $\mathrm{Ct} \leq 35$ underwent nested RT PCR targeting the capsid region followed by Sanger sequencing for genotyping of sapoviruses. Contigs were generated using Sequencher software and genotypes identified using NCBI BLAST. Out of 1266 samples, 28.44\% (360/1266) were positive for Sapovirus by qPCR. We successfully genotyped $81 \%$ (183/227) with a Ct cut off $\leq 35$. We detected sapoviruses belonging to all genogroups GI, GII, GIV and GV known to infect humans. 12 different sapovirus genotypes were detected with genotype SaV GI.1 found in $44 \%$ of all typed samples. The circulating pattern of $\mathrm{SaV}$ genotypes varied during the study period with GII.1 being predominant in 2013 and 2014 and GI.1 in 2015-2018. Sapovirus infection was detected in a considerable proportion of acute gastroenteritis cases in the setting described with an increasing trend over the years. A wide diversity of sapovirus genotypes were identified in these cases.

\section{Structure based In-silico identification of potential inhibitors of the prefusion form of Nipah virus (NiV) fusion $(F)$ glycoprotein}

\section{Murali R, Swathi Sree V, Ganesh V, Bondili JS, and Bhadra Murthy V}

Email ID for Correspondence: muralirayapati@gmail.com Genomics and Proteomics Group, Department of Biotechnology, Koneru Lakshmaiah Education Foundation (Deemed to be University), Vaddeswaram, Guntur, Andhra Pradesh, India 522502

Nipah virus (NiV) is an emerging and deadly zoonotic pathogen in the genus Henipavirus; Family: Paramyxoviridae. NiV cause acute and severe respiratory illness in humans with a high case-fatality rate more than $75 \%$. Pteropid spp. bats (flying foxes) are the reservoir hosts of NiV. Recent deadly NiV outbreak in Kerala, India during June, 2018 claimed 17 lives has put India on a high risk zone for future $\mathrm{NiV}$ infections. No vaccines or therapeutic remedies are available to prevent or treat patients exposed to NiV. NiV particles are enveloped and its genome is un-segmented negative sense singlestranded RNA of $\sim 18.2 \mathrm{kbp}$ coding six proteins. The viral envelope consists of two transmembrane glycoproteins, fusion $(\mathrm{F})$ and attachment $(G)$ glycoproteins responsible for the virus entry into host cells. $\mathrm{NiV}$ G binds to ephrin B2/B3 receptor and triggers NiV F leading to viral entry. $\mathrm{NiV} \mathrm{F}$ and $\mathrm{G}$ have been the major target of antiviral strategies to prevent viral entry. In our study, computational and cell line studies are being carried out to identify potential NiV inhibitors. Structure based In-silico analysis involving 200,000 molecules of natural origin were analyzed against pre-fusion NiV F (PDB ID: 5EVM). Two regions on NiV F (a) aa 130-190 and (b) 450-490 critical for $\mathrm{NiV}$ prefusion to postfusion transformation was targeted. Schrodinger; other suitable bioinformatics software were employed. Based on ADME parameters; tautomer conformations; PAINS; XP Docking and MDS (20 ns), seven unique molecules have been identified with high binding capabilities with at least three amino acids viz. N155; K167 and V158 of NiV F. Co-transfection 
experiments with NiV F and G plasmids on HEK-293T, Vero and $\mathrm{CHO}$ cells are being carried out to determine the extent of the inhibitory activity of these molecules in arresting syncytia formation. Promising inhibitor molecules could be used in antiviral therapy against $\mathrm{NiV}$.

\section{Anti-HIV drug Efavirenz induces cytotoxicity by initiating mitochondrial membrane depolarization}

\author{
Mandal $A^{1}$, Ganta $K K^{1}$, Chaubey $B^{1}$ \\ Email ID for Correspondence: anirbanmondal31@gmail.com \\ Functional Genomics Lab., Centre for Advanced Study ${ }^{1}$, Department \\ of Botany, University of Calcutta, 35, Ballygunge Circular Road, \\ Kolkata, India
}

Efavirenz (EFV) is a non-nucleoside reverse transcriptase inhibitor and an active constituent of the highly active antiretroviral therapy regime. It has significantly contributed in control and management of human immunodeficiency virus propagation. However, EFV administration has led to severe adverse effects, several reports highlighted the role of EFV in mitochondrial dysfunction and toxicity but the molecular mechanism has been poorly understood. In the present study, human hepatoma cells Huh-7.5 were treated with non-cytotoxic concentrations of EFV and parameters like cytotoxicity, mitochondrial transmembrane potential, mitochondrial morphology, cytochrome c release, mitochondria-mediated apoptosis, mtDNA, mtRNA levels and EFV distribution into the mitochondrial compartment were evaluated to understand sequence of events leading to cell-death in EFV-treated cells. EFV at its clinically relevant concentrations was significantly toxic after 48 and $72 \mathrm{~h}$ of treatments. The EFV-mediated toxicity is initiated with the change in $\Delta \psi \mathrm{m}$ which triggers a series of events like the cytochrome $\mathrm{c}$ release, alteration in mitochondrial morphology, mitochondria-mediated apoptosis and finally leading to cell death. We have further observed a decline in the total mitochondrial content after $48 \mathrm{~h}$ of EFV treatment at IC50 concentration which was also reflected in reduced mitochondrial DNA and RNA levels. EFV being a lipophilic molecule internalized into the mitochondrial compartment which causes depolarization of $\Delta \psi \mathrm{m}$ and subsequently leads to a cascade of events to cell death. This study underscore mitochondrial dysfunction upon EFV treatment.

\section{Sero-prevalence of dengue fever in patients with acute febrile illness in a tertiary care hospital}

\section{Dr Veenu Gupta}

Email ID for Correspondence: vsunilgupta@rediffmail.com Dayanand Medical College and Hospital Ludhiana

Fever is the commonest presentation of patients seeking healthcare in developing countries. The differential diagnosis for acute febrile illness includes malaria, dengue, enteric fever, leptospirosis, rickettsiosis, and other infections. Dengue is one of the most common mosquito-borne viral infections. Dengue has emerged as a major public health concern in terms of mortality and morbidity. The clinical and epidemiological profile of dengue infection changes from time to time. Since there is no immunoprophylaxis or specific antiviral therapy available, timely and rapid diagnosis plays a vital role in patient management and implementation of control measures. The study was conducted t to know seroprevalence of dengue fever among patients with acute febrile illness and to study their clinical and laboratory profile. A total of 6705 patients with history of acute febrile illness admitted in the hospital over a period of one year were enrolled. Serological tests like Dengue IgM and NS1 antigen ELISA were performed to confirm the diagnosis. Serologically confirmed patients of dengue fever were studied for their clinical presentation and lab parameters. The Seroprevalence of dengue fever was $17.3 \%$. The most affected age group was 26-35 years with male predominance. Most dengue cases were seen in the months of August to December. Common clinical symptoms and signs were myalgia (72.4\%), arthralgia (34.4\%), vomiting (50\%), abdominal pain $(38.6 \%)$, and hepatomegaly $(27.4 \%)$. Lab parameters revealed leukopenia and thrombocytopenia in most cases. Common complications were shock and encephalopathy. Hepatic and renal failure was seen in $3.7 \%$ and $1.8 \%$ of patients respectively. Tropical infections should be considered as important cause of acute febrile illness. Dengue fever has a very non-specific and variable presentation. Due to the overlapping clinical presentations, diagnosis must be confirmed by specific diagnostic tests.

\section{Evaluation of HiScript One-Step RT-PCR master mix with AgPath-ID One-Step RT-PCR master mix for diagnosis of influenza A (H1N1) pdm 09 virus}

\author{
Gohil $\mathrm{D}^{\mathbf{1}}$, Kothari $\mathrm{S}^{2}$, Subramanian $\mathrm{A}^{\mathbf{1}}$, Todkar $\mathbf{P}^{2}$, Khadke $\mathrm{K}^{1}$, \\ Naik $\mathbf{N}^{2}$, Warke $\mathbf{R}^{1}$
}

Department of Molecular Biology ${ }^{1}$, HiMedia Laboratories Pvt. Ltd,Department of Virology, Haffkine Institute for Training, Research and Testing ${ }^{2}$, Acharya Donde Marg, Parel, Mumbai, Maharashtra, India

Thermo Fisher Scientific's AgPath-ID One-Step RT-PCR master mix as approved by Centre for Disease Control (CDC) is used for diagnosis of influenza A (H1N1) pdm 09 virus across all nodal centres in India. The purpose of this study is to evaluate the performance of HiScript One-Step RT-PCR master mix manufactured by HiMedia Laboratories in comparison with AgPath-ID One-Step RT-PCR master mix for diagnosis of influenza A (H1N1) pdm 09 virus. A total of 50 samples were studied ( 30 positive and 10 negative retrospective cases of influenza A (H1N1) pdm 09 virus along with 10 control samples collected from healthy volunteer). Viral RNA was extracted using QIAamp Viral RNA Mini kit. One-Step Real-time PCR was performed on RNA extracted from all specimens in triplicates using both company master mixes. Applied Biosystems's CDC licensed Pandemic H1N1/09 Assay Set v 2.0 was used in this assay. HiScript One-Step RT-PCR master mix on evaluation showed better sensitivity with earlier detection of more than 1 cycle threshold $(\mathrm{Ct})$ values as compared to the AgPath-ID One-Step RT-PCR master mix. In addition, the turnaround time for diagnosis was 20 min less using HiScript One-Step RT-PCR master mix in comparison to AgPath-ID One-Step RTPCR master mix. The CDC protocol of real-time RTPCR for influenza A (H1N1) relies on limited approval of Real-time master mixes for influenza A (H1N1) pdm 09 virus diagnosis. Also, the availability and cost of these reagents is a critical element especially in resource limited countries like India. The present preliminary study provides laboratories with a more sensitive One-Step Real-time PCR master mix that can be used as a master mix of choice during the routine testing and outbreak of influenza. 
Differentiation between primary and secondary dengue virus infection by IgM: IgG ratio

\author{
Charu $^{1}$, Agarwal RK ${ }^{2}$, Mittal G ${ }^{3}$, Ahmad $S^{4}$ \\ Email ID for Correspondence: mcharukalra@gmail.com \\ Department of Microbiology ${ }^{1,2,3}$,Department of Medicine ${ }^{4}$, \\ Himalayan Institute of Medical Sciences, Dehradun, India
}

Dengue exhibits varied clinical presentations and often unpredictable clinical evolution \& outcome. More than $70 \%$ (about 1.8 billion) of the world population is at risk for Dengue. Secondary infection with the virus is an important risk factor for the development of severe forms of the disease. Methods which discriminate primary and secondary DENV infection have prognostic importance. This study was undertaken to determine the best cut off point of IgM: IgG ratio in acute phase sample and to determine the prevalent serotype of DENV in the study area. A tertiary hospital based cross sectional study was carried out and the records of 936 OPD and IPD patients suspected of dengue between Jan 2017 and Dec 2017 were collected. Dengue Duo rapid test and IgM Capture ELISA test were carried out on the blood samples. Of these, 234 tested positive for dengue either by NS1 antigen or IgM antibody testing. 91 random samples were stored at $-20{ }^{\circ} \mathrm{C}$ and further processed for IgG antibody testing by ELISA, RT PCR and Nested PCR was also performed. Of the 91 confirmed cases, $51.6 \%$ (47) turned out to be primary and $48.4 \%$ (44) secondary dengue infection. Mean IgG index and IgM: IgG index values were significantly allied with dengue illness. Best cut-off of IgM: $\operatorname{IgG}$ ratio was found to be 1.59 with sensitivity of $85.11 \%$, specificity of $100 \%$, accuracy level $92.3 \%$ and negative likelihood ratio 0.15 for differentiating primary and secondary dengue. The results of RT PCR indicated predominance of DENV 2 infection. 1.59 as a cut off for $\operatorname{IgM}$ : $\operatorname{IgG}$ ratio is recommended, a ratio of $\geq 1.59$ will imply primary dengue and $<1.59$ secondary dengue infection. DENV 2 was the most commonly associated strain with severe form of dengue in this region.

\section{Hospital based surveillance of rotavirus associated acute gastroenteritis among under five children from Haryana and Himachal Pradesh after the introduction of the rotavirus vaccine}

\author{
Gupta $\mathbf{M}^{1}$, Bansal $A^{1}$, Singh $M^{2}$, Kanojia $\mathbf{R}^{3}$, Muralidharan $\mathbf{J}^{4}$, \\ Bansal $A^{4}$, Bharti $B^{4}$, Saxena $A^{5}{ }^{5}$ Sodhi $K^{5}$, Kumar $\mathbf{R}^{1}$ \\ Email ID for Correspondence: madhugupta21@gmail.com \\ Department of Community Medicine and School of Public Health ${ }^{1}$, \\ PGIMER,Department of Virology ${ }^{2}$,PGIMER Department of Pediatric \\ Surgery,Advanced Pediatric Centre ${ }^{3}$ PGIMER Advanced Pediatric \\ Centre ${ }^{4}$ PGIMER,Department of Radiodiagnosis and Imaging 5 , \\ Postgraduate Institute of Medical Education and Research \\ Chandigarh, India
}

To monitor trend of rotavirus associated acute gastroenteritis among under five children after the introduction of rotavirus vaccine in Haryana and Himachal Pradesh, India. Hospital based surveillance system was established in a tertiary care hospital in Chandigarh, to enrol under five children admitted with acute gastroenteritis from Haryana and Himachal Pradesh, during September 2016 to May 2018, as part of a multicentric study. The rotavirus vaccine was introduced in these states in 2016. Stool samples $(5 \mathrm{ml})$ were collected, and tested for rotavirus by commercially available ELISA kits (Rotaclone; Meridian Biosciences, USA). Rotavirus positive specimens were characterized with respect to VP7 (G) and VP4 (P) proteins using reverse transcription polymerase chain reaction at Christian Medical College, Vellore. Out of.238 children (73.7\% males) enrolled $(71.8 \%$ from Haryana), 236 stool samples were collected. Rotavirus vaccine was administered to $30 \%$ of children. Rotavirus positivity rate was $21.6 \%$. Positivity was similar in both the states. The highest positivity was in the age group of $0-11$ months $(20 \%)$. The trend of rotavirus associated acute gastroenteritis was similar in summer $(21.8 \%)$ and winter seasons (21.4\%). G3P[8] (18.6\%)was the most predominant strain followed by G1P[8] (11.8\%) and G12P[6] (9.8\%). Fourteen vaccinated rotavirus positive cases, had G3P[8] (4), G1P[8] (2), G1P [4] (2), G12P[8] (2), G12P[6] (1) strains. Vaccine effectiveness was estimated to be $32 \%$ after three doses of rotavirus vaccine. There was no change in the rotavirus positivity rate $(21.6 \%)$, as earlier studies have reported positivity rate of $20.9 \%$ and $18.8 \%$ in this region. However, there is change in the most predominant genotype reported i.e., from G1P[8] to G3P[8]. Vaccine effectiveness (32\%) was lower than vaccine efficacy reported earlier from India (56.3\%) and Bangladesh $(45.7 \%)$. However, these results are from one centre, and coverage of vaccination was low $(30 \%)$.

\section{Observation and recognition of Non polio Enteroviruses from stool samples of AFP cases that produced unfamiliar cytopathic effect in L20B cell line}

\author{
Jasmeet Singh $^{1}$, Harjeet S. Maan ${ }^{1}$, N.Srivatava ${ }^{1}$, Tapan Dhole ${ }^{1}$ \\ and Rachna Chaturvedi ${ }^{2}$
}

Dept. of Microbiology Gandhi Postgraduate Institute of Medical Sciences ${ }^{1,}$ Lucknow, Uttar Pradesh, India,Amity Institute of Biotechnology, Amity University ${ }^{2}$, Lucknow

During laboratory investigation of polioviruses, stool samples producing characteristic polio-like cytopathic effect (CPE) in both L20 B cell line and RD cell line in cell culture are confirmed for polioviruses through Intratypic differentiation-real time polymerase chain reaction (ITD-rRT-PCR). However, few studies only have shown the susceptibility of L20B cell lines for infection of nonpolio enteroviruses (NPEV's). Thus, the present study involves identification of L20B cell grown isolates.

The cell culture supernatants $(n=40)$ which produced unidentified cytopathic effects in L20B cell lines and confirmed as NPEV through ITD-PCR from WHO National Polio Laboratory, Department of Microbiology, Sanjay Gandhi Postgraduate Institute of Medical Sciences, Lucknow, Uttar Pradesh, were analyzed for NPEVs.The culture suspension of these L20Bcell isolates were subjected to RNA extraction, panenterovirus $5^{\prime}$-untranslated (UTR) PCR and partial VP1 semi-nested PCR followed by sequencing and phylogenetic analysis.The Viral RNA extracted from the culture supernatants detected EV positive by Pan-EV 5'-UTR assay and further on partial VP1 gene based serotyping were identified as enterovirus types belonging to EV-A and EV-B species. A total of 18 different EV serotypes were identified, 3 serotypes such as Coxsackievirus (CV)A1, A4,A3 belong to EV-A species, whereas, 15 serotypes such as Echovirus (E)-2, 3, 5, 6, 11, 12, 20, 25, 30, 33, EVB75,EV-B93, CV$\mathrm{B} 1, \mathrm{~B} 4, \mathrm{~B} 5$ and CV-B6 belongs to EV-B species. Among the 18 serotypes CV-A $(n=10)$ and $C V-B(n=9)$ are more frequently detected than others, representing $48 \%$ of the characterized isolates. The present study concludes that besides Coxsackie-A and few of Coxsackie-B viruses, echoviruses. 
Prevalence and changing trend of viral markers (HIV, HBsAg and HCV in hemodialysis patients

\author{
Pandey $\mathbf{N}^{1}$, Mittal $\mathbf{G}^{2}$, Ahmad $\mathrm{S}^{3}$, Agarwal $\mathrm{KR}^{4}$ \\ Himalayan Institute of Medical Sciences, Swami Rama Himalayan \\ University
}

Viral hepatitis and human immunodeficiency virus (HIV) infection are important causes of mortality and morbidity in patients treated by hemodialysis (HD). Hepatitis B virus (HBV) and hepatitis C virus $(\mathrm{HCV})$ are the most important organisms responsible for the patient's morbidity. To study the prevalence of viral markers (hepatitis B surface antigen, anti HCV and HIV antibody;) in patient undergoing hemodialysis and to compare this study with a similar previous study which was conducted in our hospital 10 years back. All patients(from June 2017 to May 2018) undergoing Hemodialysis at our center were screened for hepatitis B surface antigen (HBsAg), antibody to HCV (anti-HCV) and HIV antibody by ELISA and the results were compared with a previous similar study conducted in our hospital 10 years back. A total of 463 patients (both OPD and IPD) were screened for HBsAg, HCV and HIV infections. Out of 463 patients, 253 were male and 210 were female. $13(2.8 \%)$ patients were positive for $\mathrm{HBsAg}, 35$ (7.6\%) for anti HCV and $3(0.6 \%)$ for HIV antibody. While in the previous study conducted in our hospital, there were 13 patients were HBsAg positive, 36 patients came out to be anti $\mathrm{HCV}$ positive and 2 patients were positive for HIV antibody. The prevalence of HCV is highest among all the viral markers studied. Also the prevalence of HBsAg is higher in male patients, that is statistically significant. On comparison with the previous similar study, conducted 10 years back, there is a statistically significant difference in the prevalence $\mathrm{HBsAg}$ and $\mathrm{HCV}$.

\section{Machine learning of chemically modified siRNAs and their analysis to advance RNAi based therapeutics development}

\section{Showkat Ahmad Dar and Manoj Kumar ${ }^{1}$}

Email ID for Correspondence: manojk@imtech.res.in Bioinformatics Centre, Institute of Microbial Technology, Council of Scientific and Industrial Research, Sector 39A, Chandigarh ${ }^{1}$ India

Small interfering RNAs (siRNAs) are extensively used in functional genomics and as potential therapeutics. However, they have limitations in delivery, prone to serum/cellular nucleases, target other similar sequences, etc. that can be mitigated by chemical modifications. We have already developed and published "siRNAmod an online web resource of chemically modified-siRNAs (CM-siRNAs)". Here, we implemented four methods of machine learning algorithms for model development. Random forest (RF), Linear regression (LR), Instance based learning (IBK) and Artificial neural network (ANN) were explored to predict CM-siRNA activities. 3031 CM-siRNA dataset with 30 different modifications were used for model development. Nucleotide (modification) composition, binary pattern features and their combinations were executed for tenfold cross validation and independent validation. RF achieved best performance with highest correlation of about 0.85 , while LR, IBK and ANN exhibited $0.66,0.68$ and 0.66 correlation values on training-testing dataset $\left(\mathrm{T}^{2728}\right)$ respectively. Similar performance was achieved on independent validation dataset $\left(\mathrm{T}^{303}\right)$. Subsequently, we analyzed about 150 modifications on $5000 \mathrm{CM}$-siRNAs for various chemical fingerprints. This assessment verified their tumorigenicity, mutagenicity, irritant level etc. like properties for their use in medical interventions. We concluded that multiple machine learning based models can be used to predict knockdown activity of CM-siRNAs and further chemical fingerprinting will guide for engineering their better versions for therapeutics purposes.

\section{Molecular epidemiology of influenza virus infection in Nepal}

Bimlesh Kumar Jha', A. Sapkota², S.Dangol' ${ }^{2}$,T. Shah ${ }^{2}$, B. P. Upadhyay ${ }^{1}$, K.Manandhar ${ }^{1}$

Email ID for Correspondence: bimujnp@gmail.com National Public Health Laboratory, Teku, Kathmandu, Nepal; Central Department Of Biotechnology,Tribhuvan University,(TU), Nepal

A cross-sectional study in 2018, based at National Influenza Center Nepal, was carried out with the objectives to isolate and characterize the circulating influenza viruses in Nepal. A total of 3663 throat swab specimens, obtained from patients with Influenza like Illness (ILI) at National Influenza Surveillance Network (NISN) sentinel hospitals, were transported to National Influenza Center, maintaining reverse cold chain, within $48 \mathrm{~h}$. Viral RNA was extracted using QIAmp viral RNA kit. Polymerase Chain Reaction assay (PCR) was performed following CDC Real-time rRTPCR protocol for detection and characterization of the influenza viruses including pandemic influenza virus A (H1N1) pdm 09. Randomly selected $10 \%$ of PCR positive specimens were subjected to virus isolation in Madian Darby Canine Kidney (MDCK) cells and characterized by Haemagglutination Inhibition Assay. Out of the 3663 throat swab specimens collected from ILI cases, influenza viruses were detected in 1206 (39.93\%) specimens. InfluenzaA infection was detected in 1004/3663 (11.4\%) cases; of which 948/3663 (25.8\%) were influenza A (H1N1) pdm 09 and 56/3663 (1.5\%) were influenza A/H3 subtype. Influenza B was detected in 56/3663 (1.9\%) cases. Influenza A (H1N1) pdm09 and influenza B co-infection was observed in 4/3663 (0.1\%) cases. Influenza A (H1N1) pdm 09, A/H3 and B virus were antigenically similar to the novel influenza A/California/07/2009-Like (H1N1)v type viruses; A/Victoria/361/2011 (H3N2) viruses and B/Wisconsin/ $1 / 2010$ viruses respectively. Although sporadic cases of influenza were observed throughout the year, peak was observed during July to November. The highest number of influenza A (H1N1) pdm09 and Influenza B were found in September and in children $(<15$ years of age group). All types of influenza viruses are in circulation in Nepal, with the peak during July-November. Comparison of genetic patterns of influenza virus in consecutive years is necessary to link viral genetic changes with antigenic changes.

\section{HIV transmission patterns study by using genotyping fingerprinting method among the High Risk Groups of North-West India}

\section{C.K. Chauhan, ${ }^{1}$ P.V.M. Lakshmi, ${ }^{1}$ V. Sagar, ${ }^{1}$ A. Sharma, ${ }^{2}$ S.K.} Arora, ${ }^{3}$ R. Kumar ${ }^{1,4,5}$

Email ID for Correspondence: kumar.rajesh@pgimer.edu.in ${ }^{1}$ Department of Community Medicine and School of Public Health, Post-Graduate Institute of Medical Education and Research, Chandigarh, 160012, India; ${ }^{2}$ Department of Internal Medicine, PostGraduate Institute of Medical Education and Research, Chandigarh, 
160012, India; ${ }^{3}$ Department of Immunopathology, Post-Graduate Institute of Medical Education and Research, Chandigarh, 160012, India; ${ }^{4}$ Department of Epidemiology and Population Health,The London School of Hygiene and Tropical Medicine, London, WC1E7HT, United Kingdom; ${ }^{5}$ School of Public Health and Community Medicine, University of New South Wales, Sydney, 2033, Australia

Molecular techniques for tracing of HIV transmission networks can enhance the power of epidemiological investigations. Identification of the highly related clusters by molecular methods could be used to discover sexual and injected drug users' networks. Information about the networks could be particularly useful for developing strategies to control HIV transmission among high risk groups (HRG). Hence, a study was carried out to study transmission patterns among newly diagnosed HIV cases among HRG of North-West India using fingerprinting methods. Genotyping fingerprinting method was used among 37 randomly selected samples of recently infected HRGs identified through Recent Infections Testing Algorithm (RITA) using Limiting antigen avidity assay to study transmission patterns of HIV. Amplification of the reverse transcriptase region of pol (540 base pairs) was done using Nested PCR. Sequencing was done by ABI DNA sequencer. Reference sequencing of 11 countries was extracted from HIV Los Alamos database. Alignments of sequences were done by Clustal X software. HIV-1 subtype was determined on the basis of phylogenetic analysis of the pol sequence. Phylogenetic trees were constructed using the MEGA (version 7.0). Genotype analysis of 37 recently infected HIV positive individuals revealed 6 clusters. One FSW isolate from Punjab and another FSW isolate from Himachal Pradesh showed linking with Mumbai and Pune isolates. This shows that either FSWs or their clients from North- West India move as far as up to Mumbai and Pune for work. The FSW isolates of Chandigarh, Haryana and Punjab are closely related to each other showing high movement of FSWs across these states. In men who have sex with men (MSM) two major clusters were observed. Almost all MSMs from this region belong to the same cluster showing close networking of MSMs across these states. One MSM cluster shared the same clonal clade with Pune isolate showed that the MSMs move across the country. Whereas IDU isolates shared the same clonal cluster with IDU isolates of Chandigarh, Punjab, Haryana and Himachal Pradesh only. No geographic clustering was observed from North-West and South India. None of the isolates showed interlinking with other country isolate. This shows that IDUs clusters are much closed. Molecular epidemiology methods were able to reveal transmission networks, hence these phylogenetic methods can be used to identify individuals who are within highly related transmission groups. Molecular epidemiological methods can be employed to on a sample of HIV Sentinel Surveillance to monitor transmission networks and primary drug resistance.

\section{Association of FUT2 gene with rotavirus infection among children aged 0-5 years: A study protocol}

\author{
Kaur $P^{1^{*}}$, Gupta $M^{2}$, Singh $M P^{3}$, Sagar $V^{4}$, Bansal $A^{5}$, Kanojia $R^{6}$ \\ Email ID for Correspondence: pari24k@gmail.com \\ Research Scholar, Department of Community Medicine and School of \\ Public Health ${ }^{1}$; Professor,Department of Community Medicine and \\ School of Public Health ${ }^{2}$,Professor, Department of virology ${ }^{3}$, \\ Assistant Professor, Department of Community Medicine and School \\ of Public Health ${ }^{4}$,Professor, Advance Pediatrics Center ${ }^{5}$,Professor, \\ Department of Pediatrics Surgery ${ }^{6}$, PGIMER, Chandigarh, India
}

The current understanding of the initial phase of rotavirus acute gastroenteritis (AGE) and the viral receptor is limited. Rotavirus strain specific association with ( $\alpha$-1,2-fucosyltransferase (FUT2) suggested that histo blood group antigen (HBGA) serves as an attachment factors for rotavirus. A study protocol to estimate the prevalence of secretor status and Lewis phenotype; and association of HBGA with rotavirus infection among under 5 children in North India is presented here. A cross-sectional study will be conducted among 360 under five healthy children in the field practice area of department of Community Medicine, PGIMER, to estimate the prevalence secretor status and Lewis phenotype. A test negative case-control study among 242 under five children admitted with AGE with equal number of controls in in a tertiary hospital will be conducted to estimate the association of HBGA with rotavirus infection. Rotavirus vaccination status will be obtained to find the relationship between susceptibility to infection and its impact on vaccine efficacy. Stool (10-20 g), and saliva (1-2 ml) samples will be collected from enrolled children in both the studies. Secretor status/HBGA and Lewis phenotype will be estimated from saliva samples using specific monoclonal antibodies. Fecal samples will be tested for rotavirus VP6 antigen using a commercial enzyme immunoassay Rotaclone (Meridian Bioscience) kits. Rotavirus strains will be characterized by genotyping using reverse transcription polymerase chain reaction. Odds ratio of having secretor status among rotavirus positive AGE cases as compared with rotavirus negative AGE controls, will be calculated. Estimation of odds ratio of secretor status among rotavirus positive/negative vaccinated group as compared with rotavirus positive/negative unvaccinated group will be done to comment upon the rotavirus vaccine effectiveness. The results of the study may provide evidence regarding host genetic susceptibility to rotavirus infection, and its role in vaccine effectiveness.

\section{Designing a multi-epitope based vaccine to combat human cytomegalovirus (HCMV) infection: an immunoinformatics analysis}

\section{Varun Chauhan*, Mini P Singh, Kapil Goyal}

Email ID for Correspondence: varunc1784@gmail.com Department of Virology, Post Graduate Institute of Medical Education and Research, Chandigarh, 160012

Due to the emerging evidence of drug resistance in HCMV, there is suppressing need for the development of an effective vaccine. The present study was aimed to design a multi-epitope based vaccine targeting the pentavalent complex -gH/gL/UL128-UL130-UL131 of the virus which facilitates virus entry into the host. After the application of rigorous immunoinformatics analysis and several immune filters, the multi-epitope vaccine was constructed, consisting of CD4, CD8 and B cell epitopes. The adjuvant was also linked to the epitopes in order to enhance the immunogenicity of the vaccine; all were linked via suitable linkers for effective separation in the host. Since, CMV is a double stranded DNA virus, TLR-9 will primarily recognise the structural components of CMV. Thus, $\mathrm{CpG}$ motifs were added in the vaccine construct which served as an adjuvant for triggering TLR-9 response. Further, the binding affinity and stability of the vaccine with Toll like receptor -9 (TLR-9) was analysed by molecular docking and dynamics simulation studies. In addition, an in silico cloning was performed to ensure the expression and translation efficiency of the vaccine, utilizing pET-28a (+) vector. The proposed vaccine was found to be antigenic and non- allergenic, thus ensuring its immunogenicity and safety. In addition, the epitopes included in the final vaccine construct were highly promiscuous thus could have high population coverage. Further, the molecular docking and 
molecular dynamics analysis indicated the high affinity of the vaccine with TLR-9. The in silico cloning revealed the acceptable Codon Adaptation Index (CAI) and the acceptable GC content of the optimized nucleotide sequence of the multi-epitope vaccine, thus indicating the possibility of efficient expression of the vaccine in the host (E. coli -strain K12). Such T \& B-cell-based immunotherapies which leverage this mechanism could prove their potential against HCMV infection. Further, the authors propose to test the present findings in the lab settings to ensure the safety, immunogenicity and efficacy of the presented vaccine which may help in controlling the infection.

\section{First outbreak of Chikungunya infection in Chandigarh, north India}

\section{Chhikara K, Sivanantham K, Binod Pati, Kapil Goyal, Mini P Singh, R.K. Ratho}

Post Graduate Institute of Medical Education and Research, Chandigarh, India

Chikungunya is a re-emerging infection in India since 2005 which is caused by Chikungunya virus and of public health importance with the outbreak potential as it is transmitted by Aedes mosquito vectors which are more prevalent in India and adjacent Asian countries. The suspected cases with acute onset fever or arthralgia with or without other viral prodromal features during the period of 28th of August 2016 to 25th of February 2017 from 10 states of north India around our PGIMER, Chandigarh were evaluated using serum samples in our virology laboratory for Chikungunya virus infection by IgM capture ELISA Kit (NIV Pune) specific for Chikungunya. The sero-positive cases were plotted in map manually for geographical distribution. A total of 1748 serum samples received from Chikungunya suspected patients. A total of $50.34 \%(880 / 1748)$ of suspected cases were positive for Chikungunya IgM capture ELISA. The clinical profile of available data (553/880) from Chikungunya IgM positive patients was analyzed. The maximum cases reported in the months of October and November and maximum case from Chandigarh, Punjab, Haryana $(62 \%, 22 \%, 10 \%$ of cases respectively). The cases were involved in all age group with maximum cases were distributed through $3^{\text {rd }}$ to $6^{\text {th }}$ decades of life $(13 \%, 22 \%, 28 \%, 17 \%, 10 \%$ of cases respectively) with female $(69 \%)$ preponderance of 2.23:1 ( $\mathrm{F}: \mathrm{M}=382: 171$ cases). The most common clinical feature associated with the cases are fever (97.8\%), bleeding manifestation (46.68\%), arthralgia (42.49\%), head ache $(9.4 \%)$, rash $(3.2 \%)$, myalgia $(2.7 \%)$ and thrombocytopenia $(1.6 \%)$. Data regarding duration of illness and mortality was not available. The maximum sero-positive cases were distributed around the water bodies, area with migrant and overcrowded population and construction areas of Chandigarh. This is the largest outbreak of Chikungunya in India as for our knowledge with the maximum cases in a restricted geographical region with a short span of time which emphasis the need of further surveillance and outbreak investigation.
Role of Herpes viruses as a causative agent of pneumonia in adult patient with haematological disorder Rungta $T^{1}$, Singh $M P^{1}$, Khadwal $A^{2}$, Chaudhary $P^{1}, \operatorname{Kumar} A^{1}$,
Aggarwal $\mathbf{R}^{3}$, Goyal $\mathbf{K}^{1}$, Ratho $\mathbf{R K}^{1}$

Departments of Virology ${ }^{1}$, Internal Medicine ${ }^{2}$, Pulmonary Medicine ${ }^{3}$, Post Graduate Institute of Medical Education and Research, Chandigarh, India

Viral infections remain one of the most frequent complications in patients with haematological malignancies; majority of these occur due to reactivation of latent infection. Among these infections, pneumonia due to viruses can cause significant morbidity and mortality. The aim of the present study was to find out the prevalence of pneumonia due to herpes viruses (CMV, HSV \& VZV) in patients with haematological disorders undergoing chemotherapy/immunosuppressive therapy. A total of 29 blood and bronchoalveolar lavage (BAL) samples were collected from patients (Mean age: 44.2, with M:F ratio: 2.2:1) with haematological malignancies with suspected viral pneumonia prior to the initiation of antiviral treatment. These were tested by conventional and Real Time (IVD approved) PCR targeting the immediate early gene of CMV (158 bp), glycoprotein D region of HSV (272 bp) and ORF 28 region of VZV (355 bp). Out of 29 samples, the CMV and HSV were found positive in 10/29 (34.4\%) and $4 / 29(13.7 \%)$ in BAL samples while $3 / 29(10.3 \%)$ and 2/29 $(6.8 \%)$ respectively in blood samples by conventional PCR. Similarly, by Real Time PCR, the CMV and HSV were found positive in $14 / 28$ $(50 \%)$ and $4 / 9(44.4 \%)$ in BAL samples while $4 / 25(16 \%)$ and $2 / 9$ $(22.2 \%)$ respectively in blood samples. All the samples were negative for VZV by both the techniques. Imaging findings suggestive of viral pneumonia were seen in $92.3 \%(24 / 26)$ patients which included consolidation $(30.7 \%)$, effusion $(19.2 \%)$, and nodules $(42.3 \%)$. The underlying diagnosis was AML (6/29, 20.6\%), ALL (5/29, 17.2\%), NHL and haemolytic anaemia in four $(4 / 29,13.7 \%)$ patients each, multiple myeloma $(3 / 29,10.3 \%)$, T-cell lymphoma, hairy cell Leukaemia in two $(2 / 29,6.8 \%)$ CLL, Post transplantation and myelodysplastic syndrome one each $(1 / 29,3.4 \%)$. Viral pneumonia due to herpes viruses is a serious threat for haematological malignancies. Due to similar clinical picture it becomes important to differentiate between HSV and CMV so far as the treatment is concerned. Physicians dealing with haematological malignancies should be aware of the prevalence and the causative virus in order to initiate specific antivirals in patients with high degree of clinical suspicion.

\section{Sero-prevalence of rubella among pregnant women in a tertiary care hospital, North India}

Urvashi Nehraa, Sanjay Verma ${ }^{a}$, Mini P. Singh ${ }^{\text {b }}$, R.K. Ratho ${ }^{\text {, }}$, Ravinder Kaur Sachdeva ${ }^{a}$, GRV Prasad ${ }^{c}$, Vanita Suri ${ }^{c}$

Department of Pediatrics ${ }^{\mathrm{a}}$, Virology ${ }^{\mathrm{b}}$ and Obstetrics $^{\mathrm{c}}$, Postgraduate Institute of Medical Education and Research, Chandigarh

Rubella is an acute contagious viral infection of children which is self limiting in nature. However, it is of concern when the infection occurs especially in the first trimester of pregnancy when the child can be born with congenital rubella syndrome (CRS).Successful vaccination and surveillance helped several countries to eliminate rubella/CRS. India introduced measles-rubella (MR) vaccine in 2017, and targeting children aged 9 months to 14 years. An important tool for the evaluation of vaccination programs is the serological surveillance among pregnant women which provides information about population 
immunity profiles and identifies high-risk population subgroups. The present study was conducted among 300 pregnant women in the age group of 19-45 years attending antenatal clinics in a tertiary care hospital. A maximum of 10 pregnant women were enrolled per day in order to maintain quality of data collection. Approximately $2 \mathrm{ml}$ blood sample was collected from each pregnant woman and tested for the presence of rubella IgG antibodies using commercial ELISA kit. Out of 300 pregnant women tested, 43 (14.3\%) were found to be seronegative. The majority of the sero-negative women were in the age group of 26-30 years. Nearly $4.65 \%$ women were found to be illiterate and $53.48 \%$ women were residing in rural areas. The high seroprevalence of rubella among pregnant women in absence of childhood immunization indicates continued transmission of rubella virus. However, considering that nearly $14.3 \%$ of the women are still seronegative, the introduction of rubella vaccine will be useful in the Indian scenario and will help to further lower the incidence of CRS in the country.

\section{Measles and rubella outbreaks in North India: 3 year trend}

K Sangeetha*, Sharma $M^{*}$, Sharma $V^{*}$, Rana $V^{*}$, OP Kumar*, Singh MP*, Goyal $K^{*}$, Ratho RK*, Sangal $L^{* *}$

Email ID for Correspondence: minipsingh@gmail.com *Departments of Virology, Post Graduate Institute of Medical Educationand Research, Chandigarh, India and **NPSP WHO, New Delhi

Measles and rubella are highly contagious acute exanthematous febrile illnesses, commonly occurring as outbreaks. Both these are vaccine preventable diseases. The 11 Member States of WHO SEARegion committed to eliminate measles and control CRS by 2020. The six countries to successfully eliminate measles in our region are Bangladesh, Bhutan, Maldives, Nepal, Sri Lanka and TimorLeste. This epidemiological study was conducted to analyse the trend of measles and rubella outbreaks in North India over a period of 3 years. Data from March 2015-March 2018 was collected through on-going outbreak-based measles rubella surveillance project of MoHFW under the technical guidance of WHO. Between February 2018 and March 2018, samples of case-based surveillance were also received. The surveillance areas consisted of Chandigarh and its neighbouring states. Representative samples (usually five) were received in cold chain and samples were tested as per WHO testing algorithm using commercial Siemens Enzygnost kits. A total of 471 serum samples were received for testing out of which $221(46.9 \%)$ were positive for measles. Out of 248 measles negative samples tested for rubella, $96(38.7 \%)$ were positive. Measles positivity was higher $[72.5 \%(95 / 131)]$ than rubella $[44.1 \%(15 / 34)]$ in 2015 . However, the positivity reversed in 2016 with higher rubella positivity [65\% (69/ 106)] than measles $[17.8 \%(23 / 129)]$. The trend again reversed in 2017 with higher measles positivity [35.7\% (44/123)] than rubella [13.9 (11/79)] and continued the same for the three months of 2018. Throughout the years, maximum number of outbreaks and positivity was observed during April to June. Children aged 4-9 years contributed to highest positivity for both measles (44.7\%) and rubella $(51 \%)$. The number of laboratory confirmed outbreaks decreased consecutively through all 3 years. There is a cycling trend of Measles and rubella outbreaks in North India during 2015-2018. Due to the introduction of MR vaccine, a reduction in the number of outbreaks was observed. The maximum contribution was by children aged 4-9 years, however, increased sero-positivity was observed for 9-14 years age group. This indicates the importance of introduction of second booster dose of vaccination. The recent shift from outbreak- based to case-based surveillance should be continued for marching towards the goal to eliminate measles and control CRS by 2020.

\section{Cytokine profiling in dengue patients and its correlation with dengue severity}

\section{Patil $\mathbf{S}^{1}$, Ravi $\mathbf{V}^{2}$, Desai $\mathrm{A}^{3}$}

Email ID for Correspondence: drsunipat@gmail.com

${ }^{1,2 \& 3}$ Dept of Neurovirology, National Institute of Mental Health and Neurosciences, Bangalore

Dengue fever is one of the most important mosquito borne viral infections. India is considered as hyper-endemic country for dengue. Dengue has varied manifestations ranging from subclinical infection to fatal shock syndrome. This study aimed to profile serum cytokine levels in dengue patients and correlate them with severity of dengue. We conducted cross sectional case control study of 50 serologically confirmed (NS1 Antigen/IgM antibody positive) dengue cases and 25 healthy controls over a period of 1 year. Serum levels of a total of 17 cytokines (IFN $\alpha$, IFN $\gamma$, TGF $\beta$, IL 10, IL1 $\beta$, IL 6, IL2, IL4, IL13, IL12p70, IL17 A, VEGF, GMCSF, MCP-1, TNF $\alpha$, TNFR1, TNFR2) were analysed using cytometric bead array in cases as well as controls. Clinical evaluation of enrolled 50 cases revealed, 41 cases of dengue fever, 06 cases dengue fever with warning signs \& 03 cases of severe dengue. Amongst 50 cases, NS1 antigen positivity noted in $40 \%$, IgM positivity in $2 \%$, both NS1 antigen and IgM antibody positivity noted in $58 \%$ cases. Out of the 17 cytokines analysed, mean levels of 12 cytokines (IFN $\alpha$, IFN $\gamma$, TGF $\beta$, IL 10, IL1 $\beta$, IL 6, IL2, VEGF, MCP-1, TNF $\alpha$, TNFR1, and TNFR2) were elevated in patients compared to controls. However, the levels of 5 cytokines (IL10, IFN $\alpha$, IFN $\gamma$, MCP-1 \& TNFR2) were significantly higher compared to controls ( $p$ value 0.05 ). The levels of IL10 were significantly elevated ( $p$ value 0.015 ) in severe dengue as compared to dengue fever. Levels of IL10, IFN $\alpha$, IFN $\gamma$, MCP-1 \& TNFR2 cytokines are significantly elevated in dengue patients compared to healthy controls. The levels of anti-inflammatory cytokine IL 10 is significantly elevated in severe dengue and thus maybe considered as predictor of severe dengue.

\section{Detection of multiple serotypes of dengue virus in acute febrile illness from Karnataka, India}

\section{Deepika UR ${ }^{1}$, Rai $\mathbf{P}^{1}$, Deekshit VK ${ }^{1}$, Kumar BK ${ }^{1}$, Chakraborty $A^{1}$, and Karunasagar $I^{1}$}

Email ID for Correspondence: raiprav@nitte.edu.in

${ }^{1}$ Nitte University Centre for Science Education and Research, Paneer campus, Nitte (deemed to be University), Deralakatte, Mangaluru, Karnataka, India-575018

Dengue, an important arboviral disease has become a major global concern. According to the World Health Organization report, over 3.9 billion people are at risk of dengue virus infection in 128 countries, and more than 50-100 million infections occur each year. The disease burden due to dengue infection is increasing in India, but there has been limited information on the circulating strains. Therefore, it is important to determine the serotype prevalent and circulating in a particular geographical location from time to time. The present study aims to detect the serotypes associated with the cases of acute febrile illness in Mangaluru during the period of 2018. Blood samples were collected from suspected dengue cases with febrile illness from 
Mangaluru. They were screened for NS-1 antigen of DENV, IgM, and IgG (Dengue NS-1 + Ab Combo Kit). RT-PCR was performed to detect the presence of dengue virus in serum samples using published primer pairs targeting CprM gene. Dengue virus serotypes were further characterized by nucleotide sequencing. Out of a total of 112 serum samples from suspected cases of dengue infection, $103(91.9 \%)$ samples were positive for DENV with a high prevalence of DENV-3 in $78(75.7 \%$ ) samples followed by DENV-1 in 48 samples $(42.8 \%)$. $57(55.33 \%)$ samples had co-infection with more than one DENV serotype out of which, 20 were infected with DENV-1/DENV-3, one with DENV-1/DENV-2, 11 with DENV-1/DENV-4, 5 with DENV-2/ DENV-3, and 5 samples with DENV-3/DENV-4. 16 samples showed infection with 3 serotypes. The present study provides important information on genetic diversity of the circulating dengue virus in Mangaluru, Karnataka, India. Concurrent infection with dual and triple serotypes and its co-relation with the severity was observed during the outbreak. The need for continuous monitoring of genetic diversity among dengue virus is thus important and will be useful to study disease burden.

\section{Prevalence of viral agents among cases of gastroenteritis in a tertiary hospital in northern India}

\section{Ujjala Ghoshal $^{1}$, Shikha Verma ${ }^{1}$, Juhi Sisodia ${ }^{1}$, Nidhi Tejan ${ }^{1}$, Uday C Ghoshal ${ }^{2}$}

Department of Microbiology ${ }^{1}$, Department of Gastroenterology ${ }^{2}$, Sanjay Gandhi Post Graduate Institute of Medical Sciences, Lucknow

Routinely, most of the laboratories test for bacterial and parasitic agents for diarrhoea. A high percentage of cases are clinically suspected to be of viral etiology. Multiplex PCR is a highly sensitive method for detecting the enteropathogens. Missing these leads to inappropriate patient treatment and is detrimental to infection control. Recently, few real time PCR based panels have been introduced. This study was planned to look for viral agents in diarrhoeal stool samples using Fast Track diagnostics gastroenteritis kit. To determine the prevalence of viral gastroenteritis by testing fecal samples from patients presenting with diarrhoea. Patients suffering from gastroenteritis were included in the study from August 2017 till June 2018. Demographic and clinical details of the patients were recorded in a predesigned proforma. Routine microscopy of saline and iodine mounts, modified kinyoun staining and culture were done to exclude parasitic and bacterial agents respectively. Multiplex PCR was done on extracted nucleic acids using a commercially available kit from Fast track diagnostics which detects six important viruses namely: Adenovirus, Rotavirus Norovirus GI, GII, Sapovirus and Astrovirus by Taqman technology. 70 patients suffering from gastroenteritis were included in the study from August 2017 till mid of June 2018. The mean age of patients was 45 years and male $(n=47)$ to female $(\mathrm{n}=23)$ ratio was 1.9:1. Viral pathogens were recovered in $17 \%(12 /$ 70) samples by multiplex PCR. All except one patient were from an urban background.Among patients with viral gastroenteritis, the ratio of males: females was 2.25:1(9:4).Of these Norovirus GII $(58.1 \%$; $7 / 12$ ) was the most prevalent followed by Astrovirus $(25 \% ; 3 / 12)$, Adenovirus (17.5\%; 2/12), Sapovirus (8.3\%; 1/12). Rotavirus was not found in any of the samples. A case of dual infection with Norovirus GII and Adenovirus was also detected. Abdominal pain was present in all cases of Norovirus and Adenovirus infection (100\%).Vomiting was a feature associated with all Astrovirus infections while it was absent in others except a single case of Norovirus infection. Fever was present in only one patient which was positive for Norovirus GII. The prevalence rate of viral gastroenteritis is lower than that reported from other parts of country. In this study, the highest prevalence was seen for Norovirus infections which is considered to be the second most common virus associated with acute gastroenteritis. Also, the number of children were less which may explain the absence of Rotavirus infections.

\section{Plant virology}

\section{Cotton leaf curl begomovirus complex, recombinant betasatellite and divergent alphasatellites are associated with recent outbreak of cotton leaf curl disease in Northwest India}

Kajal K. Biswas ${ }^{\mathrm{a}^{*}}$, U. K. Bhattacharyya ${ }^{\mathrm{a}}$, Supratik Palchoudhury $^{a}$, S. Godara ${ }^{a}$, S. Das ${ }^{a}$, N. Balram ${ }^{a}$, V. K. Khare ${ }^{a}$, R. Arora ${ }^{b}$ and P. K. Mandal

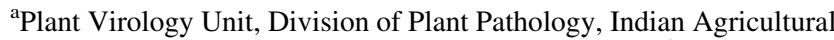
Research Institute, Pusa Campus, New Delhi 110012; ${ }^{\mathrm{b}}$ Regional Research Station, PAU, Bhatinda-151203; ' ICAR-NRCPB, LBS Building, Pusa Campus, New Delhi 110012

Email ID for Correspondence: drkkbiswas@yahoo.co.in

Cotton leaf curl disease (CLCuD), caused by whitefly transmitted monopartite begomoviruses with association of betasatellite and alphasatellites, is a serious constraint for cultivation of cotton in Northwest (NW) Indian states Haryana, Punjab and Rajasthan. CLCuD was surveyed for last six successive years from 2012 to 2017 and disease incidence was estimated. The overall incidences were $37.5,63.6,38.8,56.1,44.8$ and $58.6 \%$ in 2012 to 2017 respectively. The disease outbreak was higher of 77.5 in Haryana followed by $59.2 \%$ in Rajasthan and $54.1 \%$ in Punjab in 2013 in comparison to disease occurred in the other seasons. Infected cotton samples were collected from NW India. Thirteen CLCuD associated begomovirus (CABs) isolates were characterized based on complete genome sequencing. Complete genome was amplified through RCA, cloned, sequenced and analysed. Based on sequence analysis, eight present begomovirus sequences were found to be member of Rajasthan (Ra), one of Faislabad (Fai) and another of Pakistan (PK) strain of Cotton leaf curl Multan virus (CLCuMuV); and five sequences are member of Burewala $(\mathrm{Bu})$ strain of Cotton leaf curl Kokhran virus (CLCu$\mathrm{KoV}$ ). But CLCuMuV-Ra strain is detected as predominant strain occurring in most of the cotton growing areas of NW India. Ten present begomovirus sequences were detected as recombinants, where CLCuMuV-Ra strains are strong recombinants. Complete genome sequences of betasatellite and alphasatellite were obtained from CLCuD-affected cotton plants, sequenced and analysed. The present betasatellite sequences were found belonging Cotton leaf curl Multan betasatellite (CLCuMB) and all were recombinants. Three alphasatellite species, Cotton leaf curl Burewala alphasatellite (CLCuBuA), Gossypium darwinii symptomless alphasatellites (GDarSLA) and Croton yellow vein mosaic alphasatellite (CrYVMoA) were obtained, and most of the alphasatellites were recombinant. The present study demonstrated that the complex interaction of recombinant $\mathrm{CLCuMuV-Ra}$ strain, recombinant betasatellite CLCuMB and divergent alphasatellites is associated with CLCuD outbreak in NW India. 


\section{Biological and molecular characterization of Soybean yellow mottle mosaic virus soybean isolate from India}

\section{Sandra $\mathbf{N}^{1}$, Tripathi $A^{1}$, Mandal $B^{2}$}

Sandra Nagamani: PhD, Scientist; Tripathi A: MSc, JRF; Mandal B: $\mathrm{PhD}$, Principal Scientist

Email ID for Correspondence: Nagamani.iari@gmail.com ${ }^{1}$ Division of Seed Science and Technology, ${ }^{2}$ Advanced Centre for Plant Virology, Indian Agricultural Research Institute, New Delhi-110012, India

Soybean yellow mottle mosaic virus (SYMMV) belongs to the genus Gammacarmovirus with in the family Tombusviridae. Recently SYMMV mungbean isolate exhibiting mottling and puckering symptoms was reported from India. So, we surveyed the soybean initial varietal trials (IVT) in the experimental fields of IARI, New Delhi for the presence of SYMMV. Leaf samples collected from soybean IVT lines subjected to DAC-ELISA with SYMMV polyclonal antisera and positive samples maintained on French bean cv. Pusa Parvati by mechanical sap inoculation. Total RNA isolated from the purified virus preparation, amplified with terminal primers, cloned and sequenced. Host range study was conducted through mechanical sap inoculation on plant species belonging to families Cucurbitaceae, Leguminosae and Solanaceae and infection confirmed by DACELISA and RT-PCR with CP specific primers. Further real time PCR was performed to find out the most and least susceptible plant species. Three samples reacted positively in DAC-ELISA with SYMMV polyclonal antisera. Sequencing showed that SYMMV soybean isolate consist of $3974 \mathrm{nt}$ with six ORF's and 67 nuclotide difference from mungbean isolate. BLAST analysis showed SYMMV-Sb is $98 \%$ similar to SYMMV-Mb isolate and 76\% with SYMMV-Sb isolate of South Korea and USA. Host range studies revealed that SYMMV-Sb isolate induced veinal mottling, mild mottling and chlorotic blotching on soybean, mungbean, blackgram, cowpea and French bean at 10-15 dpi respectively. Real time PCR data showed that Pusa Parvati and Kentucky wonder were most and least susceptible from French bean, IPU and P713 was the most and least susceptible varieties of Urdbean. This study showed that Indian SYMMV-Sb isolate is $35 \mathrm{nt}$ shorter than Korean isolate and induced severe symptoms compared to Indian mungbean isolate and Korean soybean isolate. Further, this is the first report of severe strain of SYMMV-Sb from India.

\section{Biological and molecular characterization of bean common mosaic virus isolates in cowpea}

\section{B.S. Pavithra, M. Krishnareddy, Akshatha Gad, S. Jalali and D.K.Samuel}

Email ID for Correspondence: mkreddy60@gmail.com Division of plant pathology, ICAR-Indian Institute of Horticultural Research

Bean common mosaic virus (BCMV), is a species of the genus Potyvirus (family Potyviridae) is an economically important virus affecting the yield and quality of bean and cowpea due to its regular occurrence, ubiquitous seed borne nature and wide legume crop host range. BCMV isolates collected in Anndhra Pradesh, Karnataka and Tamil Nadu showed symptoms of mosaic, vein banding, leaf deformations, blistering, and caused significant yield losses (50-100\%). BCMV consists of flexuous filaments approximately $15 \times 750 \mathrm{~nm}$ containing a molecule of single-stranded, positive-sense RNA of approximately 10,000 nucleotides. The BCMV genome has a long open reading frame (ORF) and a small overlapping ORF, known as "pipo".Upon expression, a single large polypeptide is processed by three self-encoded proteases (P1, HC-Pro and NIa-Pro) to yield ten functional proteins: P1, HC-Pro, P3, 6K1, CI, 6K2, NIa-VPg, NIaPro, NIb, and CP. Widespread nucleotide variations were detected throughout the genome, with significantly more variations observed in $5^{\prime}$ UTR region. Comparative analysis of the amino acid sequences revealed that the $\mathrm{P} 1, \mathrm{P} 3$ and $\mathrm{N}$-terminal region of the $\mathrm{CP}$ proteins exhibited higher level of variations than other cistronic regions. Moreover, selection analyses further confirmed that a number of sites within the P1 and P3 genes have suffered positive selection. These obtained BCMV sequences also exhibit high recombination frequencies, indicating a more dynamic evolutionary history. Six isolates with 69 other BCMV genomes, Phylogenetic analysis showed that both soybean-infecting BCMVs (group I) and peanut-infecting BCMVs (group II) are distantly related to other BCMVs, suggesting ancestral differentiation and host adaptation. Lastly, group III and IV comprised 17 previously known BCMV sequences, with the strains in this group isolated from various plant hosts, including common bean, cowpea, mung bean, purple bush bean, peanut and also soybean. Furthermore, group I was divided into three highly supported subgroups (I-a, -b, -c and -d), which is generally in accordance with their geographic origins

\section{Sequence variability and recombination analysis of begomoviruses associated with yellow vein mosaic and enation leaf curl disease of okra in India}

\section{Zainul A. Khan, Madhvi Naresh, Indranil Dasgupta}

Email ID for Correspondence: zainulbiotechnology@gmail.com Department of Plant Molecular Biology, University of Delhi South Campus, New Delhi-110021, India

Okra/Bhendi (Abelmoschus esculentus) belonging to the family Malvaceae, is an important vegetable crop of India. India is the largest producer of okra in the world. Yellow vein mosaic and enation leaf curl disease of okra is the most important constraint to its cultivation in India. These diseases are caused by begomoviruses and associated betasatellites. Okra plants showing typical symptoms of yellow vein mosaic and enation leaf curl disease were collected from Maharashtra, Gujarat and Uttar Pradesh, India. Total genomic DNA was isolated from symptomatic and non-symptomatic okra plants. Full length genome of begomoviruses and associated satellite DNAs were amplified using rolling circle amplification kit, cloned into pGreen0029 vector and sequenced. Infectious clones of begomovirus and betasatellite were constructed and used for agroinoculation of Nicotiana benthamiana and okra plants. Sequence analysis of begomovirus isolated from yellow vein mosaic diseased samples shared maximum identity (92\%) with Bhendi yellow vein India virus (GU112050), while the sequence data of begomoviruses isolated from enation leaf curl diseased samples showed maximum identity (92-99\%) with Okra enation leaf curl virus. The full length sequence of betasatellites revealed maximum identity (99\%) with Bhendi yellow vein mosaic betasatellites and alphasatellites isolated in this study shared maximum identity (95\%) with Okra leaf curl alphasatellite. Transmission of begomovirus to host plants and putative recombination of begomovirus will be discussed. 
Betasatellite encoded $\beta C 1$ protein can hydrolyse ATP and regulates pathogenesis

\section{Gupta N, Gnanasekaran P, Chakraborty S}

Email ID for Correspondence: g.neha92@gmail.com Molecular Virology Laboratory, School of Life Sciences, JNU, New Delhi, India - 110067

In the recent years, role of betasatellites in geminivirus-disease complex has been recognized worldwide. Betasatellites are circular, single stranded DNA molecules which are associated with the monopartite begomoviruses and intensify the disease development. Betasatellites encodes a single protein, $\beta \mathrm{C} 1$ that favours pathogenesis through suppression of several anti-viral defence mechanisms in plant. Many of the viral protein shows non ATPase activity. Bioinformatics analysis revealed that $\beta \mathrm{C} 1$ possess ATP grasp motif. Protein purification was carried out by affinity chromatography followed by ion exchange chromatography. The proteins were detected through western blotting analysis using anti GST antibody. ATPase assay was done by polyethyleneimine thin layer chromatography method. To detect viral DNA level, Southern hybridization, leaf disc assay was done. In this study, the purification and biochemical characterization of $\beta \mathrm{C} 1$ protein encoded by Tomato leaf curl Patna betasatellite (ToLCPB) was carried out which exhibited a novel ATPase activity. Amino acid residue Lys-49, Arg-69 and Arg-91 are important for ATPase activity. The ATPase activity was found to influence the viral DNA and transcripts level. To know whether the ATPase activity is conserved among betasatellites, comparative assays were carried out. Nicotiana benthamiana plants infected with different betasatellite alongwith with Tomato leaf curl virus indicated differential infectivity pattern. The newly identified role of ToLCPB $\beta C 1$ mediated ATP hydrolysis regulates viral pathogenesis in plants. Critical role of the amino acids of $\beta C 1$ protein governing ATPase activity have been identified. Comparative efficacy of different betasatellites has been determined for ATPase activity and pathogenesis.

\section{Contextualizing RNAi defence response against virus infection in rice}

\section{Fauzia Zarreen and Indranil Dasgupta}

Email ID for Correspondence: fzarreen@gmail.com Department of Plant Molecular Biology, University of Delhi South Campus, Benito Jaurez Road, New Delhi-110021

Plant viruses trigger strong RNA silencing response in host plants and subsequently, the viral transcripts are targeted by the virus-derived siRNA which accumulate to high levels during virus infection. The major class of siRNA produced in response to virus infection is 21-25 nt siRNAs (VsRNA). Besides, virus infection also results in changes in the host miRNA expression profile. In course of evolution, viruses have developed strategies to counteract the RNAi-based defence of the plants. Rice Tungro disease is an important viral disease of rice affecting the rice crop in the south and south-east Asia caused by the joint infection of Rice tungro bacilliform virus (RTBV) and Rice tungro spherical virus (RTSV). With the aim of examining the plausible RNAi defence mounted against Rice Tungro infection, we analyzed the small RNA (VsRNA and miRNA) produced in rice in response to Tungro infection. Further, the RTSV genome was screened for potential RNAi suppressor. The result of this study shows that the rice RNA silencing machinery is more active against the DNA virus (RTBV) compared to the RNA virus (RTSV), both the viruses being part of the tungro virus complex. Further, RTSV proteins have very weak suppressor activity against RNA silencing, suggesting the presence of alternate silencing mechanisms. In addition, a large number of rice micro RNAs, which are crucial for the control of gene expression during various plant developmental stages are differentially regulated upon tungro infection. This study helps elucidate the elementary framework of the host RNAi defence response against Tungro infection in rice opening avenues for engineering resistance against the virus complex in rice.

\section{Topical application of double-stranded RNA molecules containing sequences of Tomato leaf curl virus and Cucumber mosaic virus provides resistance against the cognate viruses}

\author{
Namgial $T^{1,2}$, Kaldis $A^{1}$, Chakraborty $S^{2}$, Voloudakis $A^{1, *}$ \\ Email ID for Correspondence: Tsewangladakh0@gmail.com \\ Laboratory of Plant Breeding and Biometry, Department of Crop \\ Science, Agricultural University of Athens, 11855 Athens, Greece; ${ }^{2}$ \\ School of Life Sciences, Jawaharlal Nehru University, 110067 New \\ Delhi, India
}

Geminiviruses compose the largest plant-infecting group of ssDNA viruses, they are transmitted through insect vectors and infect several economically important crops causing high yield losses. Tomato leaf curl virus (ToLCV) is a bipartite geminivirus causing serious damage in tomato in India. Cucumber mosaic virus (CMV) is also a serious pathogen of tomato; CMV-infected tomato plants are stunted, bushy and may have malformed leaves and fruits. RNAi that is triggered by double-stranded RNA (dsRNA) molecules, is a powerful means to control plant viruses in transgenic plants, as well as in a non-transgenic manner in a process designated as 'RNA-based vaccination'. DsRNA molecules were made for the overlapping regions of ToLCV, namely AC1/AC4, AV1/AV2, AC1/AC4_AV1/AV2 (the latter designated as fusion construct), for CMV-2b and CMV-2b_ToLCGVAV1/AV2 (the latter designated as hybrid construct, containing regions from two different viruses). In tomato, dsRNAs for AC1/AC4, AV1/AV2, AC1/AC4_AV1/AV2 and CMV-2b_ToLCGV-AV1/AV2 conferred $45 \%, 60 \%, 50 \%$ and $55 \%$ resistance against ToLCGV, respectively. Experiments with Nicotiana tabacum showed that the dsRNA construct CMV-2b_ToLCGV-AV1/AV2 conferred $33.3 \%$ resistance against $\mathrm{CMV}$, while $\mathrm{CMV}-2 \mathrm{~b}$ provided $40 \%$ resistance. The present study also reported that the dsRNA exhibits systemic transport in tomato. This is the first case where RNA-based vaccination is functional for a bipartite geminivirus and where a single dsRNA molecule could induce resistance against two tomato-infecting viruses, namely an RNA (CMV) and a DNA (ToLCV) plant virus. Therefore, the production of single dsRNA molecules containing the desired targets could provide control against different viruses infecting the crop of interest. 


\section{Red seaweed-based formulations for effective management of plant viral diseases}

\section{Girish TR ${ }^{1}$, Sumit Bhose ${ }^{1}$, Nagaraju $\mathbf{N}^{2}$, Sri Sailaja Nori ${ }^{1}$, Shrikumar $\mathbf{S}^{1}$}

Email ID for Correspondence: girish@ sea6energy.com

${ }^{1}$ Sea6 Energy private limited, C-CAMP-NCBS, Bellary Road, Bengaluru, India 560065; ${ }^{2}$ Department of Plant Pathology, UASGKVK, Bengaluru, India 560065

Plant viruses pose a serious threat to global agricultural productivity. About $40 \%$ of the total crop loss annually is attributed to several viral diseases. Although the use of chemical pesticides has helped in managing plant viral diseases the vector control alone seems insufficient. Moreover, the indiscriminate use of chemical pesticides has resulted in undesirable consequences on both environment and human health. Therefore, the combination of vector control methods with other treatment regimens such as boosting up of plant's innate defense mechanisms using natural plant defense activators could be more effective and such a treatment regimen may also help in reducing the usage of chemical pesticides. At Sea6Energy, we aim to develop seaweed derived molecular solutions that are natural and ecofriendly. Our idea is based on the hypothesis that plants possess several layers of innate immunity that can be activated by certain seaweed derived active ingredients. Based on fractional analysis of the Kappaphycus species of cultivated red seaplant biomass, we have identified unique active ingredients that can elicit plant defense pathways. Prophylactic, foliar application of these solutions on various vegetable and horticulture crops revealed about $20-40 \%$ reduction in viral incidences and an associated overall yield improvement of up to $40 \%$. The transcriptome analysis of foliar samples treated with these formulations revealed several fold up-regulation of genes related to defense response pathways. Overall, our studies suggest red seaweed-based formulations can be effectively exploited to manage plant viral diseases.

\section{Optimization of mechanical sap transmission method on tomato (Solanum lycopersicum L.) plants with groundnut bud necrosis orthotospovirus}

\author{
Basavaraj $^{1 *}$, A. Kumar ${ }^{2}$, M.K. Yadav', V. R. Sharma ${ }^{3}$, B. \\ Mandal $^{1}$ and R.K. Jain ${ }^{1}$
}

Email ID for Correspondence: rajayb@gmail.com

${ }^{1}$ Advanced Centre for Plant Virology (ACPV), Division of Plant Pathology, ICAR-Indian Agricultural Research Institute, New Delhi110 012, India; ${ }^{2}$ Division of Vegetable Science, ICAR-Indian Agricultural Research Institute, New Delhi-110 012, India; ${ }^{3}$ Department of Genetics and Plant Molecular Biology, CSIRNational Botanical Research Institute, Lucknow, India

Groundnut bud necrosis orthotospovirus (GBNV) inciting bud necrosis disease (BND) is a major limiting virus affecting the tomato cultivation in India and Asia. Evaluation of tomato germplasm(s) for BND resistance and host-virus interactions studies became challenging due to the difficulty in inducing GBNV infection on tomato. In this study, a GBNV isolate (from tomato fruit) collected from the experimental fields and maintained on Vigna unguiculata (cowpea cv. Pusa Komal), Nicotiana benthamiana (tobacco) and Solanum lycopersicum (tomato cv. Pusa Ruby) were used as the different sources of inoculum. Performed the sap transmission on tomato seedlings from these inoculum sources with an inoculation buffer i.e. sodium phosphate buffer $0.1 \mathrm{M}$ (with $0.15 \%$ Sodium Sulphite $+0.2 \% \quad \beta$ - mercaptoethanol) prepared at four different $\mathrm{pH}$ ranges $(6.5,7.0,7.5$, 8.0). Evaluated four different growth stages of tomato seedlings viz., $15,20,25$ and 30 days after sowing (DAS) to ascertain the effective growth stage to achieve maximum rate of GBNV transmission. Conducted the transmission experiments in the environment-controlled containment facility at a temperature range of $26-28^{\circ} \mathrm{C}$. Mechanical sap transmission using systemically infected tobacco plants as inoculum source resulted in a maximum rate of transmission on tomato when inoculated with the buffer of $\mathrm{pH}$ 6.5. Most suitable growth stage of tomato seedlings for GBNV transmission was at 25 DAS, which resulted consistently the maximum percent transmission $(92-100 \%)$ in all the independent trials within 21 days postinoculation. Confirmed the GBNV infection in the inoculated plants by ELISA and RT-PCR, and noticed maximum GBNV titer in tobacco seedlings. The protocol optimized in this study will be useful in evaluating tomato genotypes for BND resistance and identifying the sources of resistance, and subsequently in breeding for BND resistance in tomato.

\section{Current status of viral diseases of cucurbitaceous crops in India}

\section{Mahesha, B. and Krishna Reddy, M}

Email ID for Correspondence: maheshpathology@gmail.com Plant Virology Laboratory, Division of Plant Pathology, Indian Institute of Horticultural Research (IIHR), Hesarghatta Lake Post, Bangalore-560089

The field survey was conducted at selective districts of Uttar Pradesh and Karnataka states (including farmer fields, Agricultural regional research stations and experimental plots) at more than 50 villages, to assess the current status of viral diseases of cucurbitaceous crops in and around the region. The major viral diseases observed on cucurbitaceous crops were belongs to the genus Begomovirus (85-100\%), Potyvirus (30-100\%), Cucumovirus (30-80\%), Tospovirus (30-100\%), Polerovirus (30-100\%), Tobamovirus (30-90\%), Ilarvirus $(40-85 \%)$ and Crinivirus $(30-65 \%)$ are most predominantly occurring in endemic manner with an incidence of $30-100 \%$ causing yield losses (10-60\%)and quality $(40-80 \%)$ loss of the produce.Among all the viruses Tomato leaf curl New Delhi virus (G: Begomovirus, F: Geminiviridae), Papaya ring spot virus-w (G: Potyvirus, F: Potyviridae), Cucumber mosaic virus (G:Cucumovirus, F:Bromoviridae), Watermelon bud necrosis virus (G: Tospovirus, F: Bunyaviridae), Groundnut bud necrosis virus(G: Tospovirus, F: Bunyaviridae), Cucurbit aphid borne yellow mosaic virus (G:Polerovirus, F: Luteoviridae), Cucumber green mottle mosaic virus(G:Tobamovirus, F:Virgaviridae), Tobacco streak virus(G: Ilarvirus, F: Bromoviridae) were predominant in cucurbit ecosystem by causing severe yield and quality loss of the economic produce. The most affected cucurbit crops are ridgegourd, pumpkin, bottlegourd, watermelon, cucumber, bittergourd, muskmelon, squash, spongegourd, snakegourd and ashgourd. All the isolates were collected and diagnosed through Transmission Electron Microscope (TEM), Atomic Force Microscope (AFM), DAC-ELISA, PCR and RT-PCR analysis with standard protocols. Further, developed the virus transmission protocols under artificial conditions and identified the host ranges of individual viruses. The mixed infections, contagiousness, seedborne nature of viruses and insecticide resistance issue, cross infections due to wide host range leads to severe and rampant spreadof cucurbit viruses. Developed the Integrated viral disease management strategies in musk melon, cucumber and ridgegourd crops that helps the cucurbit growers considerably. Further, ample scope to identify and employ the resistant sources in breeding 
programs and novel bio-technological interventions like genome editing (CRISPR/Cas9) techniques can be explored in the management of cucurbit viruses.

\section{Reverse transcription-loop mediated isothermal amplification: a rapid diagnostic assay for detection of Potato virus $A$ in potato and aphids}

\author{
Baswaraj Raigond $^{* 1}$, Verma $A^{1}$, Pathania $S^{1}$, Jandrajupalli $S^{2}$, \\ Verma $\mathbf{G}^{\mathbf{1}}$, Kochhar $\mathrm{T}^{\mathbf{1}}$ and Chakrabarti SK ${ }^{1}$ \\ Email ID for Correspondence: raigond@gmail.com \\ ${ }^{1}$ ICAR-Central Potato Research Institute, Shimla, Himachal Pradesh- \\ 171001, India; ${ }^{2}$ ICAR-National Institute of Biotic Stress \\ Management, Raipur, Chhattisgarh- 493225, India
}

Potato virus $A$ is an important virus infecting potato, transmitted through infected tubers, vegetative planting material and by aphids. It's one of the major concerns for raising healthy seed potato. Under field conditions, window available for monitoring viral incidence, monitoring of viruliferous aphid etc., is minimal. Hence, draw special attention for developing rapid and sensitive diagnostic assay to taking-up timely management practices. Hence, we aimed to develop LAMP assay for specific detection of PVA. Total RNA isolation and RT-PCR based confirmation of PVA from pure culture and from experimentally prepared viruliferous aphids was conducted. The LAMP assay was optimized and confirmed its specificity and sensitivity. Extended for detection in tubers and in single aphids and validated by running across field collected potato and aphid samples. A RT-LAMP assay was optimized where, $61^{\circ} \mathrm{C}$ for $60 \mathrm{~min}$ gave sharp ladder like amplification and total volume of the reaction mixture was successfully reduced to $10 \mu \mathrm{l}$. Assay was visualized by naked eyes by adding SYBR gold nucleic acid stain. It was found to be highly specific as there was no cross reaction with other viruses infecting potato. Sensitivity of the assay was equivalent to RT-PCR, were it was able to detect the virus at $10^{-2}$ dilution. The assay can be successfully applied for detection of PVA in potato tubers. Squash print RT-LAMP assay was developed and found suitable to determine viruliferous nature of aphids. Finally, the optimized RT-LAMP and SP-RT-LAMP assays were successfully validated by running across potato samples and aphid vectors collected randomly from fields respectively. Developed RT-LAMP and SP-RT-LAMP assay for specific and rapid detection of PVA in potato plants and in single aphids respectively which can assist in raising healthy seed potato.

\section{A comprehensive study on resistance to tomato leaf curl virus against various combinations of $\mathrm{Ty}$ genes in tomato}

\author{
Pardhasaradhi $\mathbf{P}^{1,2}$, Rakesh Kumar*1, Sairam $\mathbf{P}^{\mathbf{1}}$, Ramachandran \\ $\mathbf{E}^{1}$, Santanu Acharya ${ }^{1}$ and Rajashaker $\mathrm{P}^{2}$ \\ Email ID for Correspondence: pardhasaradhi@jkagri.com \\ JK Agri Genetics Ltd, Hyderabad; ${ }^{2}$ JNTU, Hyderabad
}

Tomato Leaf Curl Virus (ToLCV), a begomovirus, is a major constraint for the production of tomato cause up to $100 \%$ crop loss. To overcome these problems, researchers identified Ty-genes from wild tomato species. In this study, we introgressed these genes into commercial tomato lines and tested its efficacy against begomoviruses. ToLCV symptomatic samples were collected and isolated its genomic DNA. These samples were tested in PCR with universal primer, Deng $\mathrm{A} / \mathrm{B}$ and confirmed the presence of begomovirus. However to identify viral strain, designed a specific primer, (TL12F/ 13R for mono and TL14F/15R for bipartite) using DNA-A genome of ToLCV. The amplified PCR products were sequenced and submitted in NCBI database. The samples amplified with primer TL12F/13R similar with tomato leaf curl Karnataka virus (Acc.No KY311884) and with TL14F/15R similar with Tomato leaf curl New Delhi virus (Acc.No. MH269415). These strains were maintained in greenhouse on tomato plants through whiteflies transmission. These strains were tested on breeding population with different combinations of Ty genes (Ty-1, Тy-2, Тy-3, ty-5, Тy- $1+$ Ty-2, Тy $2+$ Тy 3 , Тy $2+$ ty5 , Ty $1+$ Ty 3 , ty-5 + Ty- 6 and Ty- $1+$ Ty- $2+$ Ty-3) along with susceptible control. The healthy whiteflies incubated on tomato plant infested with either mono or bipartite for $24 \mathrm{~h}$ then released on experimental tomato plants. After infestation, the data was recorded on 20 th and 45 th day using $0-4$ disease scoring. The results after 45 days against monopartite showed that plants having Ty- $2+$ Ty- 3 and Ty- $1+\mathrm{Ty}-2+\mathrm{Ty}-3$ were tolerant. However, against bipartite begomovirus, plants having Ty $2+$ Ty 3 and Ty- $1+$ Ty- $2+$ Ty- 3 were moderately tolerant after $20 \mathrm{dpi}$ and became susceptible after 45 dpi. The Ty genes interrogation into commercial tomato lines is useful to alleviate losses due to ToLCV.

\section{Understanding the role of glycine rich RNA binding proteins in viral pathogenesis}

\section{Email ID for Correspondence: ragunathan.sci@gmail.com}

Glycine rich proteins (GRP) represent a group of proteins, which are characterized by the conserved semi-repetitive glycine rich motif at its C-terminal. GRPs are involved in growth, development and other multiple cellular processes. GRPs are also induced during various abiotic and biotic stresses but the mechanism during biotic stress especially during viral pathogenesis is poorly observed. Structurally, the Class IV GRPs essentially consist of two domains, a structured N-terminus containing an RNA Recognition Motif (RRM) and a flexible C-terminus with numerous glycine making the part intrinsically disordered. Although key reports are available about the role of Class IV GRPs in biotic stress responses, a role of these proteins in the life cycle of viruses has not yet been intensively studied.

NbGRP3 transcript level in viral infected samples quantified by northern hybridization/qRT-PCR. NbGRP3 cloned and purified to study the interaction with viral DNA using EMSA/fluorescence binding. NbGRP3 (a Class IVa GRP from $N$. benthamiana, a model organism for plant-virus interaction studies) shows elevated transcript levels upon infection of Geminiviruses, i.e., Tomato leaf curl New Delhi virus (ToLCNDV) along with non-cognate Radish leaf curl betasatellite $(\operatorname{RaLC} \beta)$ in $N$. benthamiana. Similar kinds of observations were obtained with Tomato bushy stunt virus (TBSV), which is an RNA virus. Upon further investigation, it was found that NbGRP3 interacts with Satellite Conserved Region (SCR) of Radish Leaf curl virus Beta satellite. Up-regulation of transcript level of NbGRP3 upon viral infection (both DNA and RNA viruses) and their interaction with viral DNA stress on a role of NbGRP3 during viral pathogenesis. Yet further experiments need to be performed to understand the precise role of NbGRP3 in viral pathogenesis. 


\section{Characterization of begomovirus and associated satellite components causing leaf curl disease in Alcea rosea $\mathrm{L}$}

\section{Kumar Manish, Kumar RV, Chakraborty S}

Email ID for Correspondence: mannuranchi@gmail.com Molecular Virology Lab, School of life sciences, Jawaharlal Nehru University, New Delhi-110 067, India

Hollyhock (Alcea rosea L. family Malvaceae) is an annual ornamental plant grown in tropical, subtropical and temperate regions of the world. Geminiviruses infect a large variety of crop plants around the world and cause a new threat to global food security. Earlier studies reported that begomoviruses are known to infect hollyhock plants. In February 2016, Begomovirus-like symptoms were noticed on the hollyhock plants grown in a garden at the campus of JNU, New Delhi. Since the hollyhock plants exhibited typical symptoms of yellow vein mosaic, we anticipated the possibility of association of begomoviruses in these plants. Rolling circle amplification was performed to clone the associated begomoviral genomic components from the total genomic DNA isolated from the infected hollyhock plants and clones were sequenced. The phylogenetic analysis was carried out by MEGA-X software and the recombination analyses of the cloned begomoviral genomic components were analyzed using (Recombination detection program) RDP. We have identified a new virus species, Hollyhock yellow vein virus: New Delhi and isolates of Cotton leaf curl Multan virus, Ludwigia leaf distortion betasatellite, Cotton leaf curl Multan alphasatellite and Sida leaf curl virus alphasatellite in association with yellow mosaic disease. Recent studies also suggest that the begomoviruses have been derived through extensive recombination processes. The study suggests that the occurrence of recombination events between related begomoviruses may lead to the appearance of the new begomovirus species. We identified a new virus species, Hollyhock yellow vein virus: New Delhi along with the others begomoviral genomic components, infecting the hollyhock plants. The infectivity of hollyhock yellow vein virus in different host plant needs to be assessed to confirm the agro-economic threat of the begomoviruses.

\section{Identification of Brassica yellows virus, a Polerovirus in Radish (Raphanus sativus)}

\section{Email ID for Correspondence: suvi.ss519@gmail.com}

Survey conducted in the vegetable growing regions of Karnataka indicated incidence of virus and virus like diseases with symptoms of yellowing, chlorotic lesions, interveinal chlorosis and leaf distortion in radish. The symptomatic samples collected from field, were washed with RNase-free sterile double distilled water, sap extracted and were examined under Electron microscope, which showed the presence of isometric particles of $28-30 \mathrm{~nm}$. Total RNA were extracted from symptomatic leaf samples and healthy control were used for RT-PCR amplification using Polerovirus specific generic primers. The results have shown amplification of 350 bp DNA fragment from infected samples but not from healthy control plant samples. Cloning, sequencing and nucleotide blast search showed 93\% similarity with poleroviruses and more than $93-99 \%$ with Brassica yellows virus (BrYV) (Genus: Poleroviruses; Family: Luteoviridae). This is the first report of the occurrence of BrYV in radish in India. BrYV has a wider host range and is an emerging viral disease with potential threat. Further complete genome sequence is under progress.

\section{Identification of differentially expressed small RNAs following Tomato leaf curl virus infection in N. benthamiana}

\section{Email ID for Correspondence: vedjiwan@gmail.com}

The phenomenon of RNA silencing protects host from viruses and in addition can also silence endogenous heterochromatin and transposons, transcriptionally or post-transcriptionally in a sequencespecific manner1, 2. The trigger for this process are double stranded RNA (dsRNA) precursors. DICER-LIKE (DCLs) proteins in plants, which are RNase III-type endoribonucleases, cleave dsRNA into short RNA duplex of 21-24 nucleotide in length3, 4. Subsequently, the produced small RNAs (sRNAs) are loaded onto RISC complex. While interacting with RISC, the passenger strand is degraded while the guide strand binds to the complementary sequences in the target mRNA leading to either cleavage of the mRNA transcript or translation inhibition. RNA dependent RNA polymerases (RDRs) are the enzymes in plants which uses primary small-interfering RNA (siRNA) produced by DCLs, to generate longer dsRNA to be processed into secondary siRNA. The secondary siRNA amplifies the signal leading to the systemic silencing. Many plant hormones are also regulated by a variety of WRKY and other proteins. We have inoculated $N$. benthamiana plants and collected leaf samples ( $21 \mathrm{dpi}$ ) to generate sRNA libraries. We have uses next generation sequencing (NGS) to identify differentially expressed sRNAs in N. benthamiana following Tomato leaf curl virus (ToLCV) infection. Several sRNAs were found to be differentially expressed. Among them genes encoding WRKY-55, Cytochrome P450 like TBP, Laccase, Serine/ threonine-protein phosphatase and Phospho-pantothenoylcysteine decarboxylase etc. were up-regulated following virus infection in $N$. benthamiana. In contrast, genes encoding Photosystem II CP43 chlorophyll apoprotein, Pyridoxal phosphate (PLP)-dependent transferases, Photosystem II CP47 chlorophyll apoprotein, Integrin-linked kinase-associated serine/threonine phosphatase $2 \mathrm{C}$ were down-regulated. The in silico analysis suggests ToLCV infection results in differential expression of sRNAs. Among the sRNAs, those regulating transcription factors or various enzymes might be involved in plant metabolic pathways.

\section{Development of chimeric promoters from parareterovirus for strong expression of ectopic gene in plant system}

\section{Dipinte Gupta* \& Rajiv Ranjan}

Email ID for Correspondence: guptadipinte90@yahoo.in Plant Biotechnology Lab, Department of Botany, Faculty of Science, Dayalbagh Educational Institute (Deemed University), Dayalbagh, Agra 282005, India

Promoters are specific sequence of nucleotides present upstream of the gene coding region; comprises of multiple cis-acting elements having specific binding for transcription factors which are involved in initiation and regulation of transcription. The basic strength of promoter lies in the notation and arrangement of these cis-acting elements and their combinational interaction with transcription factor. 
Hereby-strong promoters can be developed by redesigning their architecture, which can be exploited for strong expression of ectopic gene. In the present study, eight chimeric promoters were synthesized by using the two well-established approaches: domain swapping and hybridization. Promoters from Rice Tungro Bacillus Virus (RTBV) and Mirabilis Mosaic Virus (MMV12) were used as parents for the development of these chimeric promoters. All the developed promoters were cloned upstream of aGUS reporter gene and their efficacy was tested by transient as well as transgenic assay. Gus assay of electroporated tobacco protoplast and agro-infilterated tobacco, petunia, rice and millets was performed for transient expression studies. Transgenic lines of tobacco were generated at $\mathrm{T}_{0}$ stage for the expression profiling of developed promoters. By doing the expression profiling on biochemical, histo-chemical and molecular level, twoout of the eight developed promoters, designated as REM and RTBVP1MMV12 were found to have great efficiency. On comparing these developed promoters with the most widely used promoter CaMV 35S, $18.12 \%$ and $32.8 \%$ increase in expression was found in REM and RTBVP1MMV12 respectively in dicots while in monocots $30 \%$ and $98.64 \%$ increasewas found in REM and RTBVP1MMV12 respectively. From the obtained results it might be concluded that we developed promoter can be efficiently used for translational research in plants and can pave the way off for plant biotech industries.

\section{Development of efficient synthetic Promoter from plant parareterovirus}

\section{Dipinte Gupta\& Rajiv Ranjan}

Email ID for Correspondence: guptadipinte90@yahoo.in Plant Biotechnology Lab, Department of Botany, Faculty of Science, Dayalbagh Educational Institute (Deemed University), Dayalbagh, Agra 282005, India

Promoters are specific sequence of nucleotides present upstream of the gene coding region; comprises of multiple cis-acting elements having specific binding for transcription factors which are involved in initiation and regulation of transcription. The basic strength of promoter lies in the notation and arrangement of these cis-acting elements and their combinational interaction with transcription factor. Hereby-strong promoters can be developed by redesigning their architecture, which can be exploited for strong expression of ectopic gene. In the present study, eight chimeric promoters were synthesized by using the two well-established approaches: domain swapping and hybridization. Promoters from Rice Tungro Bacillus Virus (RTBV) and Mirabilis Mosaic Virus(MMV12) were used as parents for the development of these chimeric promoters. All the developed promoters were cloned upstream of aGUS reporter gene and their efficacy was tested by transient as well as transgenic assay. Gus assay of electroporated tobacco protoplast and agro-infilterated tobacco, petunia, rice and millets was performed for transient expression studies. Transgenic lines of tobacco were generated at $\mathrm{T}_{0}$ stage for the expression profiling of developed promoters. By doing the expression profiling on biochemical, histo-chemical and molecular level, twoout of the eight developed promoters,designated as REM and RTBVP1MMV12 were found to have great efficiency. On comparing these developed promoters with the most widely used promoter CaMV 35S, $18.12 \%$ and $32.8 \%$ increase in expression was found in REM and RTBVP1MMV12 respectively in dicots while in monocots
$30 \%$ and $98.64 \%$ increasewas found in REM and RTBVP1MMV12 respectively. From the obtained results it might be concluded that we developed promoter can be efficiently used for translational research in plants and can pave the way off for plant biotech industries.

\section{Identification of chilli (Capsicum annuum L.) lines with enhanced resistance to cucumber mosaic virus under controlled and field conditions}

\section{Kavyashri V V, Nagaraju N and Mohan Rao A}

Email ID for Correspondence: kavyashrivv@gmail.com

Cucumber mosaic cucumovirus (CMV) is a destructive pathogen with a wide host range. Identification of resistant sources and understanding the genetic behavior of resistance is essential for resistance breeding. One hundred and eleven chilli lines collected from public and private institutions were evaluated for CMV resistance both under field and greenhouse conditions. Reaction of chilli lines for CMV was visualized through symptoms and confirmed by Double Antibody Sandwich Enzyme-Linked Immunosorbent Assay (DAS-ELISA). The CMV disease incidence (DI) was ranging from 0.0 to $100 \%$, six chilli lines viz., AVPP 0906, AVPP 1110, Aparna, Susanjoy, Phule Jyothi and ADR Driver were remained free from CMV infection with least disease progress (AUDPC) therefore catalogued as highly resistant. Rest of the lines exhibited characteristic CMV symptoms. Twelve lines namely, AVPP 0302, AVPP 0508, AVPP 1111, A. Khyati, Assam 2, HMT 1, Byadagi dabbi, Ujwala, LCA 960, AVPP 0904, Wakako long and Utkal Rashmi were found resistant and moderately resistant reaction both under field and greenhouse conditions. However under mechanical inoculation, six lines were categorized as highly resistant, nine as resistant, three as moderately resistant, 19 as moderately susceptible and 74 as susceptible. Whereas under field condition, six lines showed highly resistant reaction to CMV, five lines showed resistant reaction, eight lines were moderately resistant, three lines were found moderately susceptible, ninety one lines were found susceptible to CMV based on DI. Differential responses of CMV severity might be due to vector load, climatic conditions and genetic characteristics of chilli lines. These highly resistant and resistant lines could be used by breeders in developing new chilli hybrid resistant to CMV.

\section{Emerging virus and phytoplasma diseases in Rajouri District of Jammu and Kashmir}

\section{Mohd Ashaq}

Email ID for Correspondence: ashaqraza@gmail.com Higher Education Department, J\&K Govt

Climate change has triggered a number of plant viral diseases in recent past which were never observed or recorded before. There is urgent need to detect these newly emerging diseases and provide control measures to ensure the better plant heath hence greater food security. In this connection, a random survey was conducted to study the virus and phytoplasma infecting important vegetables, fruit plants, ornamentals and weeds in District Rajouri of Jammu and Kashmir State (India). Vegetables like potato, chili, turnip, radish, cabbage, cauli- 
flower, knoll kohl, peas, okra, brinjal, and cucurbits (bottle gourd, cucumber, snake gourd and bitter gourd etc.), wild fruiting plants like figs and blackberries, ornamentals (wild rose, marigold, Catharanthus etc.) and weed hosts were surveyed. In many plants, severe effect on plant health and crop losses due to virus and phytoplasma infections was observed. Several virus infected samples were collected for further biological and molecular-based investigation. Different kinds of virus or virus-like symptoms like mosaics, vein clearing, vein banding, ring spot, leaf curling, leaf rolling etc. in different crops/plants were observed. During survey, in many cases, different kinds of symptoms in infected plants were observed, which were not recorded previously, it might be due to the effect of climate change that influences the pathogens to become aggressive and more virulence or forcing to shift to the new hosts. Initial field surveys have been conducted and samples are collected for further investigation. Diagnostic techniques for virus identification include 1. biological properties related to the interaction of the virus with its host and/or the vector (symptomatology and transmission tests) and 2. intrinsic properties of the virus itself (coat protein and nucleic acid). Detection methods based on coat protein include precipitation/agglutination tests, enzyme-linked immunosorbent assays, and immunoblotting whereas viral nucleic acid-based techniques are like dot-blot hybridization assays and polymerase chain reaction (PCR). The results are awaited as initial survey has been completed and molecular detection is going on. The survey of virus and phytoplasma diseases on virus various plant species provide a clue on the impact of climate change on plant health may open new vistas for investigation and controlling plant diseases.

\section{First report of Soybean Yellow Mottle Mosaic Virus on Glycine max in India}

\section{Email ID for Correspondence: tripathiankita2302@gmail.com}

Soybean yellow mottle mosiac virus (SYMMV) belongs to the genus Carmovirus with in the family Tombusviridae has been recently reported from mungbean in India. Later, while surveying for other leguminous hosts of SYMMV in the fields of Indian Agricultural Research Institute, New Delhi, three soybean samples strongly reacted with the polyclonal antibodies raised against SYMMV in direct antigen coating ELISA (A405 $\mathrm{nm}=1.2-1.8$ ). The total RNA from the positive leaf samples was isolated and tested through RT-PCR with the coat protein (CP) specific primers NS1 and NS2 which the amplicon of $1065 \mathrm{bp}$. The PCR product was further cloned in TA vector, confirmed by restriction digestion, sequenced and submitted to NCBI data base (MG768974). Sequence analysis of the CP gene showed 98-100\% identity with Indian isolates (KR260903, KR260902, and KP259608) followed by 82 and $92 \%$ similarity with Korean isolates of SYMMV at nucleotide and amino acid levels, respectively. For further confirmation mechanical sap inoculation was performed on French bean cv. Pusa Parvati with an extract from ELISA- positive soybean leaf sample which showed the chlorotic blotches at 10-12 days post inoculation. These symptomatic leaves tested and confirmed for SYMMV with DAC ELISA and RT-PCR with CP primers. Previously,SYMMV was reported on soybean from South Korea and North America More recently, SYMMV was reported on mungbean and urdbean from India. To our knowledge, this is the first report of natural infection of soybean by SYMMV in
India and suggests that this newly identified SYMMV may be adapting to other leguminous host plant species.

\section{Development of gene deletion or point mutation based constructs of croton yellow vein mosaic virus to understand their suitability as replicon vector}

\section{Gurpreet Kaur, BikashMandal and Anirban Roy}

Email ID for Correspondence: 22gurpreet1990@gmail.com Division of Plant Pathology, Indian Agricultural Research Institute, New Delhi - 110012

It has been shown that genome of a plant virus can be modified in a way so that it can efficiently replicate in the plant without producing symptoms or producing mild symptom. Hence, such modified virus genome based replicon can be used as a vector to carry a gene/gene fragment and deliver that into plant. Such vectors are extremely useful to express any foreign protein in plant or to study functional behavior of any plant gene. Recently, such plant virus based vectors werealso employed to deliver genome editing tools inside the plant. To utilize a plant virus as a replicon vector it is essential to know the minimal replicon size that allow their replication and systemic movement inside the plant. With an aim to develop such plant virus based replicon vector, genome of a begomovirus(ssDNA), Croton yellow vein mosaic virus(CYVMV) was modified and different gene deleted and point mutant constructs were made from the infectious construct of CYVMV using PCR and cloning strategy. Besides that another construct containing the intergenic region, which has the origin of replication was made in pGreen II vector in tandem duplicates separated by a multiple cloning site with 6 restriction enzyme sites. All these constructs were transformed first into DH5 $\alpha$ cells, and then electrotransformed in EHA105 strain of Agrobacterium tumifacience with pSoup helper plasmid. To evaluate the constructs they wereagroinfiltrated into Nicotianabenthamiana plants and the replication behavior of these constructs and symptom phenotype produced by themwere assessed. Such begomovirus based replicon vector will open up a new era for effective utilization of plant viruses as a gene delivery tool for plant.

\section{Synonymous codon usage pattern of Citrus tristeza virus reveals high codon adaptability to Citrus reticulata, an evolutionary primitive citrus host}

\author{
Supratik Palchoudhury, Shibu Das, Prosenjit Chakraborty \\ and Kajal Kumar Biswas
}

Email ID for Correspondence: supratik88pc@gmail.com Advanced Centre for Plant Virology, Division of Plant Pathology, ICAR-Indian Agricultural Research Institute, New Delhi-11012, India

Citrus tristeza virus (CTV), a member of the aphid transmitted Closterovirus, is the causal agent of the notorious Tristeza disease in several citrus species worldwide. The codon usage patterns of viruses reflect the evolutionary changes for optimization of their survival and adaptation in their fitness to the external environment and the hosts. The codon usage adaptation of CTV to specific citrus hosts has not 
been studied, thus its role in CTV evolution is not comprehended clearly. Therefore, to better explain the host-virus interaction and evolutionary history of CTV, the codon usage patterns of the coat protein $(\mathrm{CP})$ genes of $122 \mathrm{CTV}$ isolates originating from three economically important citrus hosts (55 isolate from Citrus sinensis, 38 from Citrus reticulata and 29 from Citrus aurantifolia) were studied using several codon usage indices and multivariate statistical methods. The present study shows that CTV displays low codon usage bias (CUB) and higher genomic stability. Neutrality plot and relative synonymous codon usage analyses revealed that the overall influence of natural selection was more profound than that of mutation pressure in shaping the CUB of CTV. The contribution of high frequency codon analysis and codon adaptation index value shows that CTV has host-specific codon usage patterns resulting in higher adaptability of CTV isolates originating from $C$. reticulata ( $\mathrm{Cr}-\mathrm{CTV})$, low in the isolates originating from $C$. aurantifolia (Ca-CTV) and $C$. sinensis (Cs-CTV). The combining codon usage analysis of CTV with citrus genealogy suggests that CTV has evolved in C. reticulata, an evolutionary primitive Citrus species or other Citrus progenitors. The outcome of the study enhances the understanding the factors involved in viral adaptation, evolution and fitness towards their hosts. This information will definitely help to devise better management strategies of CTV.

\section{Development of single-tube multiplex polymerase chain reaction assay for simultaneous detection of $\mathrm{C}$}

\section{Email ID for Correspondence: vivekkhare.19@gmail.com}

Cotton leaf curl disease (CLCuD) caused by whitefly-transmitted monopartite $(\sim 2.7 \mathrm{~kb}$ DNA-A) begomoviruses in association with beta- and alpha-satellites ( $\sim 1.4 \mathrm{~kb}$, both), is a serious constraint in entire cotton growing area of 1.1 Mha of Haryana, Punjab and Rajasthan in Northwest (NW) India. Betasatellite affects disease complex and pathogenicity by suppression of host gene silencing, whereas, alphasatellite can reduce virus titre, preferentially betasatellite accumulation, and can attenuate disease symptoms. Cooccurrence of the DNA-A and betasatellite molecules in CLCuD infected cotton synergistically enhances disease symptoms. For detection of the virus and its associated satellite molecules, a quick, sensitive and cost effective detection method is required. With this objective a single-tube multiplex polymerase chain reaction (mPCR) assay was developed for simultaneous detection of CLCuD-begomovirus (DNA-A), and its associated beta- and alpha-satellite molecules. Several sets of primers for amplification of the virus and satellite molecules based on the retrieved reference sequences from the GenBank were designed and tested considering various parameters, annealing temperature and template and primer concentration. Of them, three sets of primers targeting (i) complete coat protein (CP) gene (750nt) of CLCuD begomovirus, (ii) complete $\beta C 1$ gene (363nt) of betasatellite and (iii) partial Rep gene (445nt) of alphasatellite were standardized and used in simplex (s) and multiplex (m) PCR. The constant amplified products were identified on the basis of amplicon size in $\mathrm{mPCR}$ and were compared with sPCR. The amplicons were cloned and results were confirmed by sequence analysis. The optimized mPCR protocol was validated by testing infected cotton samples exhibiting variable symptoms collected from different areas of NW India. The mPCR will definitely save time and reagent costs for diagnostics. This tool can be used for screening disease resistance, quick diagnosis, virus indexing and epidemiological studies.

\section{Characterization of Candidatus Phytoplasma australasiae-related strain associated with big bud and witches-broom disease of Tomato and Carrot in India based on the studies of 16S rRNA and SecY gene}

\section{V.Venkataravanappa*, P. Swarnalatha and M. Krishna Reddy}

Email ID for Correspondence: venkatrajani@gmail.com Dr.V.Venkataravanappa, Scientist (Plant Pathology), Division of Plant Pathology, Central Horticultural Experiment Station (CHES)Chettalli-571248; ICAR-Indian Institute of Horticultural Research (IIHR), Bangalore Karnataka, India

Introduction: Tomato and carrot is one of the important vegetable crops grown throughout the country under diverse agro climatic conditions. The crop is prone to many fungal, bacterial, phytoplasmal and viral diseases. Among these, big bud and witches-broom disease of tomato and carrot caused by phytoplasma is a looming threat for tomato and carrot cultivation in India. Tomato and carrot plants showing big bud/witches broom were collected from farmer field were identified based on the PCR using phytoplasmic-specific primer pairs of 16S rRNA and SecY gene. The amplified PCR product was cloned and sequenced. The complete nucleotide (nt) sequence of 16S rRNA and SecY genes of tomato big bud and carrot witches broom phytoplasmas isolates showed maximum nt identity more than 95\% with Candidatus Phytoplasma australasiae (16SrII). Further In-silico RFLP analysis of 16SrRNA gene of tomato big bud samples showed similarity coefficient in the range of $0.68-0.95$. Whereas carrot witches broom isolates showed similarity coefficient 0.93 . Therefore the strains are significantly distinct from other subgroups of Candidatus Phytoplasma australasiae. Further, the phylogenetic analysis revealed that, they are closely clustered with Candidatus Phytoplasma australasiae strains (16Sr II), specifically within the 16Sr II-D and 16Sr II-A subgroups. The study highlights the identification of new strains of phytoplasmas associated with tomato and carrot in India. The association phytoplasma is one of the most important alarm signal for its production and serious threat cultivation these crops.

\section{Role of proteasomal pathway gene(s) in combating virus infection in plants}

\section{Manoj Prasad}

Email ID for Correspondence: manoj_prasad@nipgr.ac.in National Institute of Plant Genome Research, Aruna Asaf Ali Marg, JNU Campus, New Delhi

Tomato leaf curl disease (ToLCD), caused by strains of Tomato leaf curl virus (ToLCV), is a major constraint to tomato production. To understand the molecular mechanism of virus tolerance, we studied the abundance of viral genomic replicative intermediate molecules and virus-derived siRNAs generated by host plant in naturally tolerant (H-88-78-1) and susceptible (Punjab Chhuhara) cultivars at different days-post-infection. We showed that less abundance of viral replicative intermediate in tolerant cultivar may have a correlation with a relatively higher accumulation of virus-specific siRNAs. Further, a significant correlation was observed between siRNAs accumulation and altered methylation patterns in tolerant versus susceptible cultivars. These suggest that both viral DNA methylation and siRNA-mediated degradation play an important role in conferring 
tolerance against ToLCNDV. Further, we functionally characterized a (i) $26 \mathrm{~S}$ proteasomal subunit RPT4a (SIRPT4) gene and (ii) U-box type E3 ligase (SlARM18) gene, which were differentially expressed after ToLCNDV infection in tolerant cultivar. (i) The study showed that SIRPT4 protein binds to promoter region of ToLCNDV genome thereby hindering the expression of virus genes which subsequently reduces viral replication and infection in tolerant cultivar. Transient overexpression of SIRPT4 resulted in activation of programmed cell death and antioxidant enzymes system. (ii) An increase in viral load upon silencing SlARM18 in cv. H-88-78-1 was observed validating its role in tolerance. Upon virus infection, WRKY41 was found to regulate the expression of SlARM18 by binding onto the W-box elements present within the promoter of SlARM18. Further, downstream, SIARM18 interacts with viral AC4 protein and leads to its ubiquitinylation. Overall, present study highlights non-proteolytic function of SIRPT4 as well as SIARM18-mediated ubiquitination of viral AC4 protein and their participation in defense-pathway against virus infection in tomato.

\section{Detection of six important begomoviruses and two different betasatellites in chilli from Gujarat}

Email ID for Correspondence: jainaga.p@gmail.com

Zairah W, Pradeep Kumar, Anirban Roy, Bikash Mandal

Chilli leaf curl has emerged as a serious viral disease in the cultivation of chilli in the different parts of India. In this study, nineteen chilli samples (ChGj1-19) showing leaf curl and yellowing symptoms were collected from the farmer's fields at Surat, Gujarat, India were analysed. In order to know the begomoviruses and their betasatellite associated with the leaf curl disease, PCR was conducted using degenerate as well as specific primers. The ten samples those showed positive with degenerate primers were further amplified using six begomovirus species specific primers [BM796F \& BM797R for tomato leaf curl Bangalore virus (ToLCBaV); BM800F \& BM801R for tomato leaf curl Gujarat virus (ToLCGuV); BM802F \& BM803R for tomato leaf curl Joydebpur virus (ToLCJV); BM798F \& BM799R for tomato leaf curl New Delhi virus (ToLCNDV); BM794F \& BM795R for tomato leaf curl Palampur virus (ToLCPalV) and BM861F \& BM862R for chilli leaf curl virus (ChiLCV)]. The PCR with the species specific primers revealed prevalence of all the above begomoviruses and importantly showed the association of multiple begomoviruses up to five different begomoviruses in a single plant sample. All the nine positive samples analysed for the detection of betasatellite using universal beta primers (CLB36F \& CLB37R). The five samples (ChGj14, 15, 16, 17 and 19) that showed the positive amplification with

\section{Molecular screening and characterization of weeds as reservoirs of Begomoviruses around leguminous crops}

\author{
Sudeep Pandey ${ }^{1}$, Nagaraju, $\mathbf{N}^{2}$ and Girish $\mathbf{T R}^{3}$ \\ Email ID for Correspondence: psudeep12@gmail.com \\ ${ }_{1,2}$ Department of Plant Pathology, University of Agricultural \\ Sciences, GKVK, Bengaluru-65; ${ }^{3}$ Sea6Energy Private Limited, \\ C-CAMP,NCB-GKVK Campus, Bengaluru-65
}

Yellow mosaic disease (YMD) caused by Begomoviruses is a critical constraint in the production of leguminous crops. A season-long survey was carried out for YMD in six different leguminous crops and in associated natural weeds, across different regions of southern Karnataka during 2017 and 2018. As high as about 94.0 per cent of YMD incidence was recorded across six leguminous cropping systems surveyed. Among the 96 weed samples surveyed 54\% were symptomatic while $45.8 \%$ were asymptomatic. Both symptomatic and asymptomatic weed samples were PCR screened using Deng primer, Mungbean yellow mosaic virus and Horsegram yellow mosaic virus Coat protein gene specific primers. Among the asymptomatic weed samples 55.20 per cent amplified through direct PCR, while in 18.75 percent samples the PCR amplification was observed only after the enrichment of the template through Rolling circle amplification. Out of the nine weed species commonly observed the weeds, Agertum conyzoides, Alternanthera sessilis, Commelina benghalensis and Euphorbia geniculata were found abundantly. Further, sequence analysis of PCR products amplified from weed samples revealed a close clustering with the Horsegram yellow mosaic virus isolateLima bean, Legume yellow mosaic virus and Mungbean yellow mosaic virus isolate Nammakal, Moreover, a cross infectivity test on blackgram and soybean crops using viruliferous whitefly, Bemisia tabaci that were fed respectively on weeds A. conyzoides and E. geniculate revealed clear YMD symptoms. Overall, these data suggests a potential role of weed species on the epidemiology of the Begomoviruses in legume crop systems and thus useful towards developing future virus management strategies.

\section{Development of binary vector constructs with genes of Indian Citrus Ringspot Virus toward identifica}

\section{Email ID for Correspondence: aniketa19@gmail.com}

Kinnow orange is one of the widely accepted fruit due to its vitamin $\mathrm{C}$ rich high juice content. It is cultivated mainly in Punjab areas of India and has high export values. In the recent past, Indian citrus ringspot virus (ICRSV) is reported to be widely-distributed and known to infect Kinnow affecting qualitative and quantitative yield losses up to $100 \%$. The infected trees produce the typical symptoms such as rings, vein-clearing, vein-banding flecking and chlorotic pattern on the mature leaves. ICRSV is classified into genus Mandarivirus and family Flexiviridae. It contains a single-stranded + sense RNA genome size of $7.5 \mathrm{~kb}$ long which consist six open reading frames (ORFs). Identification of ICRSV suppressor protein(s) which is able to interrupt the host defense by RNAi mechanism is required to understand the virus-host interaction. In this study, we collected the infected and healthy kinnow leaves based on typical symptoms from the ICRSV Punjab isolates maintained in glass house of IARI, New Delhi. Total RNA from leaves were isolated using TRIzol reagent. Two-step RT-PCR was performed with the gene specific primers containing the restriction sites targeting to clone into pCAMBIA binary 1302 vector. PCR amplified ORF2 of 678 bp, ORF3 of $330 \mathrm{bp}$, ORF4 of 183 bp and ORF6 of 669 bp of ICRSV was cloned in binary vector pCAMBIA 1302. Positive clones were selected and DNA sequenced. The DNA sequences were analyzed to be $99 \%$ identical with the previous isolates of ICRSV. The positive binary vectors will be mobilized into Agrobacterium tumefaciens strain GV3101 and agroinfiltration study will be performed in GFP expressing Nicotiana benthamiana $16 \mathrm{c}$ line to identify the suppressor protein. 
WRKY41 mediated transcriptional regulation of a $\mathrm{U}$ box type E3 ligase SlARM18 leads to targeting of AC4 protein of Tomato leaf curl New Delhi virus

\section{Arunava Mandal, Namisha Sharma, Priya Dulani, Shambhavi Sharma \& Manoj Prasad}

Email ID for Correspondence: namisharma.ns@gmail.com National Institute of Plant Genome Research, Aruna Asaf Ali Marg, JNU Campus, New Delhi

Ubiquitination is a post-translational modification involved in various developmental processes and stress responses in plants. SIARM18 (Ubox type E3 ligase) was found upregulated ( fivefold) during ToLCNDV infection in tolerant tomato cultivar. Given this SIARM18 was characterized for its role in tolerance against ToLCNDV. Silencing of SIARM18 was performed through VIGS. For promoter analysis deletion promoter constructs were generated in fusion with GUS-GFP reporter gene. EMSA was performed to validate DNAprotein interaction. Yeast-two hybridization and BiFC were performed to identify interacting proteins. Ubiquitinylation assay was performed to validate ligase activity of S1ARM18. Increase in viral load upon silencing SlARM18 in cv. H-88-78-1 was observed validating its role in tolerance. Histochemical and fluorometric GUS assay revealed involvement of two W-box elements within promoter of SIARM18 in tolerance response. In-vitro DNA-protein interaction by gel shift assay indicates strong interaction between WRKY41 and W-box elements present in the SlARM18 promoter. SlARM18 was found to interact with AC4, viral protein and deletion of U-box domain abolished this interaction. In-vitro and in vivo Ubiquitinylation assay indicated that SIARM18 comprises a strong ligase activity and it ubiquitinates viral AC4 protein. Further AC4 was found to be the substrate for ubiquitinylation by SlARM18 leading to its degradation via proteasome system. The present study provides novel clues on role of SIARM18 in conferring tolerance against ToLCNDV. SIARM18 ubiquitinates viral AC4 and it could be adopted as a strategy for developing viral resistant plant by overexpressing SlARM18.

\section{Association of a Nucleorhabdovirus with vein clearing disease of cardamom in India}

\section{Bhat AI, Biju CN, Pamitha NS}

Email ID for Correspondence: aib65@yahoo.co.in ICAR-Indian Institute of Spices Research, Kozhikode 673012, Kerala, India

The vein clearing (kokke kandu) disease caused by an unknown virus is an important production constraint of the cardamom crop in India. The disease is characterized by chlorosis of the veins followed by rosetting, loosening of leaf sheath and shredding of leaves; newly emerging leaves get entangled in the older leaves and form hook-like tiller and hence the disease is locally known by the name kokke $k a n d u$. The infected plants decline rapidly with yield reduction up to $62-84 \%$ in the first year of peak crop and perish within 1-2 years of infection. In the present study causal virus associated with the disease was successfully transmitted through aphid, Pentalonia caladii. Small RNA (sRNA) sequencing of the aphid transmitted cardamom plant showed several contigs aligning with nucleorhabdoviruses. Two regions of the causal virus covering 9806 nucleotides was amplified through RT-PCR using primers based on the sequence of the contigs that mapped to the nucleorhabdoviruses. Cloning and sequencing of the amplified product showed identities ranging from 30 to $62 \%$ in the nucleocapsid $(\mathrm{N})$, phosphoprotein $(\mathrm{P})$, putative movement protein (P3), matrix protein $(\mathrm{M})$, glycoprotein $(\mathrm{G})$ and polymerase $(\mathrm{L})$ genes of different nucleorhabdoviruses thus confirming the identity of the virus as a distinct species under the genus, Nucleorhabdovirus for which the name Cardamom vein clearing virus is proposed.

\section{Characterization, in silico prediction of begomovirus infected papaya (Carica Papaya) encoded microR}

\section{Email ID for Correspondence: abhibiot2k4@gmail.com}

Leaf curl disease of papaya is caused by several species of Begomovirus, family Geminiviridae. The leaf curl disease of papaya is transmitted by whitefly (Bemisia tabaci), and is single stranded monopartite in nature; however bipartite genome is also reported. The disease is characterized by downward curling and cupping of leaves followed by vein thickening. Every year, India witness tremendous loss to its papaya growing areas. Infected leaves were collected from papaya plants from Delhi and its suburbs during 2016 and 2017 and virus DNA were cloned and characterized. Agroinfectious clones were also developed. Bioinformatics study throws light on the similarity and dissimilarity between the different isolates of papaya leaf curl virus that has infected papaya plant in various regions of India and sub continents. MicroRNAs (miRNAs) belong to class of endogenous small RNAs which suppress expression of genes following cleavage or translational inhibition of target messenger RNAs. They are involved in a number of plant processes such as development, biotic and abiotic stresses. In this study, in silico approach is employed, high scoring miRNA-target pairs satisfying rules of minimum free energy and maximum complementarities were selected. This study shows predicted papaya miRNAs which have the potential to target different genes, including AC4 region, which involve in gene silencing suppression. Further next generation sequencing data reveals several new miRNA from infected papaya leaf sample.

\section{Solanum whitefly Aleurothrixus trachoides (Back) can transmit Geminivirus?}

\section{K. Chandrashekar, Ashutosh Rao, Savarni Tripathi and Rajverma}

Email ID for Correspondence: Kc_shekar2001@yahoo.com ICAR-IARI, Regional Station, Pune

Solanum whitefly Aleurotrachelus trachoides (Back). (Hemiptera: Aleyrodidae) is reported to occur on tomato, capsicum, eggplant, tobacco, Duranta $s p$ and several other weeds. The pest is native to the neotropics and presently reported to occurs in several countries. In India it was first reported by Dubey and Sundararaj during 2015 from Karnataka and they described it as Aleurothrixus trachoides (Back). The damage caused by A. trachoides is mainly due to feeding on plants sap. In the year 2017 European and Mediterranean Plant Protection Organization declared A. trachoides as a non vector. However, infected plants show typical virus infection symptoms hence, the present study was undertaken to identify the possible occurrence of geminiviruses. Samples of A. trachoides and its infested host plants Duranta sp. tomato, capsicum, eggplant, Solanum nigrum and Ipomoea sp. were collected from Pune, Maharashtra. DNA was extracted from plant samples and from individual whiteflies. The isolated DNA of whitefly and plant were tested for occurrence of Geminivirus using 
degenerate primers. PCR analysis using degenerate primers for geminiviruses showed approximately 520 base pair amplicons as expected in 12 out of 20 Duranta $s p$ samples and 6 out of 40 whitefly samples collected from Duranta $s p$. None of the other samples showed any amplification. The results indicated the presence of Geminivirus in Duranta $s p$ and whitefly by degenerate primers and RCA amplification. Further studies to characterise geminivirus transmission ability of A. trachoides is under progress. The results suggest the presence of geminivirus in whitefly A. trachoides as well as in its host plant Duranta $s p$. Hence, there is a need for detailed studies to understand and determine vector status of A. Trachoides.

\section{Effects of biotic stress on the essential oil yield and quality of Mentha spp. and screening diseas}

\section{Email ID for Correspondence: birendrak67@rediffmail.com}

Background Mentha (family Lamiaceae) consists of different species/varieties based on flavor, and among them, three species of mints (Mentha arvensis, M. piperita, and M. spicata) are commercially cultivated in India for the essential oils. It is widely used in various pharmaceutical and confectionary industries. Since last decade apart from abiotic stress (water, salinity, light), biotic stress caused negatively influence the survival, biomass production, oil yield, and quality. Comparative studies are reported here. Materials and Methods Different species of Mentha were studied in the experimental field of CSIR-CIMAP, Lucknow with reference to their health, productivity, quality product and were also screened for the selection of improved genotype tolerant to fungus and virus infection. Conventional and molecular approaches were applied to develop genotype with required characters. Essential oil extraction was carried out using Clevenger apparatus and further subjected for gas chromatography analysis to compare the yield and quality of oil in the healthy and infected Mentha spp. Results Leaf curling, as well as necrotic spots and patches, were found on the leaves of infected $M$. arvensis, $M$. spicata, and $M$. piperita. PCR results showed the presence of viral and fungal infection. The main oil constituents were identified as limonene, menthone, menthofuran, menthol, carvone and methyl acetate. The chemical profile of $M$. arvensis oil showed an increase in menthol content and decrease in limonene percentage in infected plant in comparison to healthy while $M$. piperita showed increase in menthol content accompanied by an increase in menthofuran. Some accessions/lines are identified as tolerant to fungal/viral infection for the detailed studies. Conclusion Phyto-pathogens (viruses and fungi) are responsible for the stress, cause changes in the quality and quantity of the essential oil. Therefore to combat the effect of infection caused by fungi and viruses, vigor genotype via breeding program is needed.

\section{Molecular characterisation of Begamovirus associated with a new viral disease on Clerodendrum serrat}

\section{Email ID for Correspondence: karmveergautam09@gmail.com}

Clerodendrum serratum (L.) Moon. (Verbenaceae) is an important medicinal plant growing in the tropical and warm temperate regions like Africa, Southern Asia; Malaysia and distributed throughout in the forests of India and Sri Lanka. It is traditionally valued and reported for treating pain, inflammation, rheumatism, respiratory disorders, fever and malaria in India with a long history. During routine field survey at CSIR-CIMAP in April 2018, Clerodendrum serratum have been found to be suffering from green mosaic and blistering on leaves as compared to healthy plants. To ascertain the presence of the begomovirus, total DNA was isolated from twelve symptomatic and one asymptomatic leaf samples of $C$. serratum by the CTAB method and subjected to PCR using begomovirus $\mathrm{CP}$ specific primers (GemF/ $\mathrm{R})$. Further, the full-length genome was amplified by the RCA method and digested by different restriction enzymes. Obtained bands were cloned and sequenced and sequences were analyzed by bioinformatics tools. Based on the visual virus-like symptom, about $30 \%$ of plants have been found to be symptomatic. Virus-like symptoms and presence of the whitefly population, a Begomovirus infection was suspected. PCR result showed $\sim 771$ bp amplification in all symptomatic leaves (twelve) samples. PCR result supports the presence of the Begomovirus infection. Digested RCA product produced $\sim 2.7-$ $\mathrm{kb}$ band. Analysis of the sequence data showed $99 \%$ identities, and close phylogenetic relationships with the DNA-A genome of Tomato Chilli leaf curl India virus (ToChiLCuIV) (FM877858) reported from India. Based on high sequence identities and close relationships, the begomovirus isolated from $C$. serratum was identified as an isolate of (ToChiLCuIV) infecting C. serratum from Lucknow, India. In the literature, there is no disease has been reported on $C$. serratum so far. We, here, have reported Tomato Chilli leaf curl India virus infecting C. serratum for the first time in India.

\section{Molecular characterization, pathogenesis and recombination studies of rapidly evolving Begomoviruses}

\section{Email ID for Correspondence: asifakhan103@gmail.com}

Andrographis paniculata (Kalmegh) is a medicinal herb, well known for its antioxidant, antidiabetic, anticancerous, anti-inflammatory, antimicrobial and hepatoprotective properties. Begomovirus is the largest genus of the family Geminiviridae, infecting a large range of plants. Recombination is one of the major factors for the emergence of new species of begomoviruses and the expansion of its host range. Although few fungal infections are reported, no viral infection had been reported earlier on it. Our study is the first report of viral infection on $A$. paniculata. During the survey of the Kalmegh growing areas, typical symptoms made the preliminary detection of the viral infection. PCR performed using begomovirus coat protein specific primers for the molecular detection of the virus. Full-length genome, amplified using PCR and RCA techniques, was sequenced and characterized. Agrobacterium-mediated transformation was used to check the molecular infectivity of the virus in different hosts. Recombination studies were analyzed using bioinformatics tools. Typical virus-like symptoms such as leaf curling, vein clearing, yellowing were noticed on the infected plants of A. paniculata. Full length genome corresponding to $2.7 \mathrm{~kb}$ was obtained by PCR and RCA methods. Another genomic component of $1.3 \mathrm{~kb}$ corresponding to betasatellite was also detected. The complete genome was sequenced and submitted in GenBank database. Based on the nucleotide sequences, they were identified as Eclipta yellow vein virus (Acc no. KC476655), Papaya leaf crumple virus (Acc no. KM359408) and a new species of Begomovirus named Andrographis yellow vein leaf curl virus (Acc no. KM359406). Molecular infectivity assay revealed the presence of betasatellite, and it helped in symptom enhancement. Recombination events detected in all these three virus isolates. The cultivation of A. paniculata is not sufficient 
to meet the demand of pharmaceutical industries. Heavy loss of such medicinally important crops by begomoviruses should be managed.

\section{Mapping and characterization of begomovirus betasatellite transcripts}

\author{
Reddy $K^{1}$, Chakraborty, $S^{1}$
}

Email ID for Correspondence: kishore.bios7@gmail.com

${ }^{1}$ Molecular Virology Laboratory, School of Life Sciences, Jawaharlal Nehru University, New Delhi-110067, India

Begomovirus associated betasatellites encodes for single multi-functional protein $\beta \mathrm{C} 1$, which plays a key role in betasatellite-mediated pathogenesis. Mutagenic studies on betasatellite speculated the origin of different transcripts other than predominant $\beta \mathrm{C} 1$ transcripts. The current study attempted to identify and characterize the putative transcripts originating from Radish leaf curl betasatellite (RaLCB$\mathrm{R} \beta$ ) upon co-inoculation with Tomato leaf curl New Delhi virus (ToLCNDV-NA). Transcript mapping studies were performed by northern blotting, primer walking and random amplification of cDNA ends (RACE). RNA gel blots of RNA extracted from NA + R $\beta$ infected plants identified an uncharacterized transcript from the satellite conserved region (SCR) and the expression of the transcript enhanced as the disease progressed. Primer walking identified multiple transcripts of various lengths ranging from 94 to $913 \mathrm{bp}$ across the SCR and $\beta C 1$ ORF. Since geminiviruses are reported to have bidirectional promoters, RACE experiments were carried out for virion and complementary sense strands to further identify the nucleotide coordinates of transcription start and end points. $5^{\prime}$ and $3^{\prime}$ RACE uncovered a transcript on virion strand $(\beta \mathrm{V} 1)$ between 234 and 584 nucleotides with potential ORF coding capacity. Transient expression of $\beta \mathrm{V} 1$ induced potential hypersensitive response and peroxides accumulation followed by cell death in $N$. benthamiana plants. The $\beta C 1$ transcript mapping revealed the ORF with a $5^{\prime}$ UTR of $615 \mathrm{bp}$ and $3^{\prime}$ polyadenylation signal $33 \mathrm{bp}$ downstream to the ORF. Betasatellite transcript mapping identified multiple polyadenylated transcripts. $\beta \mathrm{C} 1$ and putative $\beta \mathrm{V} 1$ ORF genes mapped and putative $\beta$ V1 ORF induces HR-mediated cell death.

\section{Analysis of susceptibility to RNMV infected plants in response with M. oryzae and X.Oryzae, Xanthomonas oryzae pv. Oryzae and CMV}

\section{Wagh, S. G. Shamim, A. Daspute, A., Bhor, S. A., Kobayashi, K., and Nishiguchi, $M$}

Email ID for Correspondence: swagh.gene@gmail.com MGM, IBT, Aurangabad, INDIA

Rrice necrosis mosaic virus (RNMV) causing mosaic symptoms characterized by yellow flecks and streaks on lower leaves of rice plants was first reported in Japan and then in India (Fuji, 1877, Ghosh, 1980, Badge et al. 1997). This virus has been classified as a member of the Bymovirus genus of the Potyviridae. RNMV is transmitted by a fungus, Polymixa graminis. RNMV inoculated by growing rice seedling in soil infested with the fungal vector Polymixa graminis carrying RNMV. Wild type rice (Oryza sativa L. cv. Nipponbare) and the transgenic lines were used in this study. Oryza sativa cv. Nipponbare is susceptible to both $X$. oryzae pv. oryzae and $M$. oryzae. The seeds of Asominori and Sensyou, which are resistant to $X$. oryzae pv. oryzae and M. Oryzae. We examine that RNMV virus infected plant were again inoculated individually with pathogens Magnaporthe oryzae, Xanthomonas oryzae pv. Oryzae,Cucumber mosaic virus. After inoculation lesion size analysed in both Magnaporthe Oryzae and Xanthomonas oryzae pv. Oryzae. Among them Magnaporthe oryzae shown higher infection level, where $C M V$ and Xanthomonas oryzae pv. Oryzae were moderate.The similar results confirmed by analyzing bacterial biomass content and fungal DNA content, in case of CMV purified virus inoculated, RNA content analyzed by q-RTPCR. RNMV virus has moderate effect on yield of rice, but it may have role and in resistance breakdown and facilitation to divesting pathogens such as Magnaporthe Oryzae shown in this study. These findings indicate that RNMV infection alters plant immunity which facilitates the other fungus and virus infection.

\section{Animal virology}

\section{Vaccines and vaccination concepts in bluetongue}

\section{A.B. Pandey}

Division of Biological Standardization, Indian Veterinary Research Institute, Izatnagar

Bluetongue (BT) is a culicoides transmitted economically important disease of domestic and wild ruminants. In India, disease was first reported in 1964, since then several outbreaks have occurred and it is now endemic in this country. In India, sheep is the main species affected with the disease in the frequent outbreaks causing huge mortality. Other animals like goat, cattle, buffalo, antelope, dear, sambhar, camels and llamas also get affected by/infected with the virus. Disease can take course of asymptomatic to lethal forms depending upon the BTV serotype, species, breed and age of animal involved. Disease causative agent is BT virus (BTV), belonging to the Orbivirus genus of Reoviridae family. In sheep, the disease varies from acute to chronic forms and sometimes there is subclinical infection. Affected sheep have fever, depression, anorexia, tachypnea, hyperemia of the lips and nostrils, salivation, nasal discharge, edema of the face and tongue, conjunctivitis, ulcers on oral mucosa, rarely, cyanosis of the tongue, coronitis, laminitis, muscle weakness, lameness, consequently, animal stand with an arched back, dermatitis, torticollis and wool breaks. Gravity of the disease problem can be understood from the fact that 22 , out of 27 serotypes known worldwide, are circulating in ruminants in India, which has varied climatic conditions across the length and breadth of the country having competent vectors for disease transmission. Because of a large number of susceptible hosts, BTV serotypes and Culicoides vector, control of BT is not an easy task. The control strategy of bluetongue can be successful mainly through vaccination of animals with vaccine using right serotype of the virus. Different regions of the country, depending upon the number of virus serotype(s) prevalent in particular region will require the vaccine prepared from those respective serotype(s). An ideal BT vaccine is efficacious, safe, affordable, protective against multiple serotypes and enables the differentiation of infected from vaccinated animals. It is difficult to prepare an ideal vaccine, however, a judicious balance will have to be made between efficacy and duration of immunity, safety for the animal and the environment, costs, applicability for multiple serotypes, and possibility for DIVA. Both the Live attenuated vaccines and inactivated vaccines have been successfully used in China, South Africa, Europe and other countries. A genetically engineered virus-like particle (VLPs) has also been projected as a next generation vaccine. However, prevalence of multiple serotypes within a limited geographical area and incidence of genetic and phenotypic drift during natural infection in vectors and hosts, generating neutralization-resistant 
phenotypic variants within a serotype make the circumstances further difficult. In India, attempts have been made to develop inactivated BTV vaccines using BEI and Hydroxylamine. BEI inactivated BTV vaccine elicited both neutralizing and group specific antibodies whereas hydroxylamine inactivated vaccine elicited group-specific antibodies. Both the inactivated vaccines showed reduction in clinical signs and duration of viremia. An inactivated pentavalent vaccine (BTV-1, 2, 10, 16 and 23) has been developed and commercialized in the country. Modified live vaccines (MLVs) produce a viremia in animals which lead to further spread of the viruses and reassortment of their genome. Sheep rearing by the nomadic communities which are always on move will further restrict the use of MLVs. Many new vaccine candidates, ranging from subunits to replicating next-generation reverse genetics based vaccines, have been developed with their merits and demerits and therefore the latest ones are yet to come to market. Hence, in Indian scenario, use of multivalent inactivated vaccines is a good option for control of disease. Regular monitoring of the serotypes of the virus circulating in the different parts of the country, their isolation and identification to ascertain the serotype and to include it/them in monovalent/multivalent vaccine formulation will be proper strategy for successful control of the disease. Various approaches to develop BT vaccines and issues of vaccination will be discussed.

\section{Japanese encephalitis: a zoonotic threat and our preparedness}

\author{
Ashok Kumar ${ }^{1}$, Himani Dhanze ${ }^{2}$ and Chethan Kumar ${ }^{3}$ \\ ${ }^{1}$ Indian Council of Agricultural Research, New Delhi, India; ${ }^{2}$ ICAR- \\ Indian Veterinary Research Institute, India; ${ }^{3} \mathrm{ICAR}^{-}$Central Coastal \\ Agricultural Research Institute, Goa, India
}

Japanese encephalitis (JE) is a mosquito-borne zoonotic flaviviral disease responsible for causing hundreds of fatalities in children every year in India. The virus is maintained in complex cycle in nature wherein egrets and herons act as reservoir hosts, mosquitoes of genus Culex as vector, swine as amplifier host and human beings as dead end host. Based on nucleotide sequences of envelope gene, JEV has five genotypes (I-V) and only one serotype. All known JEV strains isolated in India until 2007 belonged to genotype III. Later, the presence of genotype I in 2009 in Gorakhpur region (Uttar Pradesh, India) and subsequently in 2010 in West Bengal has also been reported. Swine being an amplifier host plays an important role in epidemiology of JE and considered as a significant risk factor for transmission of virus to human beings. Seo-conversion in swine occurs $2-3$ week before infection comes to human beings and hence swine act as a suitable sentinel for prediction of JE outbreaks in human population. Recently, researchers have found vector free transmission of JEV in experimental pigs through oro-nasal route though it is yet to be proven in natural field conditions. Though reported for the first time in 1955 in India, JE emerged as a major public health problem in India since 1973 when first major outbreak took place in West Bengal and later the deadly outbreaks in year 1978 and 2005 in Gorakhpur region of Uttar Pradesh. Earlier maximum cases were reported from Uttar Pradesh, Assam, West Bengal and Bihar. Presently, JE virus has spread its realm to various other states of India where earlier it was not reported. Several factors including climate change may be responsible for its spread. Government of India has included vaccination against JE as part of the routine immunization under the Universal Immunization Program in 206 JEendemic districts in the country. However, still there is ample scope to increase vaccination coverage in the country. Animal husbandry sector is actively involved in surveillance of JE in swine population particularly in endemic states like Uttar Pradesh. Screening of more than 3000 swine serum samples by ICAR-Indian Veterinary Research Institute, Izatnagar from across the country revealed a sero-positivity of 32 per cent. IVRI has also developed indigenous diagnostics for the detection of JE infection in swine. Indigenously developed diagnostics and vaccines for human $\mathrm{JE}$ are also available in our country. However, existing infrastructure facilities i.e. diagnostic laboratories for the routine surveillance in human and veterinary field need to be scaled up. The sentinel surveillance must start throughout the country so that outbreaks in humans are timely predicted and prevented. In addition, expansion of vaccination program, hospital upgradation and sanitation in rural and urban areas along with sentinel swine surveillance are key elements in control and prevention of JE. Human vaccination against JE has dramatically decreased incidence of JE and hence sustained efforts are required to strengthen and expand JE vaccine coverage. Inter-sectoral collaboration between human health, animal husbandry and other ministries to have a common platform for surveillance and control program ensures better preparedness. Because of climate change, changing vector competence, shift in genotype of circulating virus, a complete relook into the epidemiology of JE is the need of the hour. The time has come to adopt One Health approach to control this disease in our country.

\section{Current status of Peste des petits ruminants vaccination programme in India}

\section{Balamurugan, G. Govindaraj, K.P.Suresh and Parimal Roy}

Email ID for Correspondence: balavirol@gmail.com; b.vinayagamurthy@icar.gov.in Indian Council of Agricultural Research-National Institute of Veterinary Epidemiology and Disease Informatics (ICAR-NIVEDI), Post Box No. 6450, Yelahanka, Bengaluru - 560064, Karnataka, India

Peste des petits ruminants (PPR), otherwise known as "goat plague" or "Small ruminant plague" is enzootic in India as number of outbreaks have occurred in the past and now being occurring regularly, round the year and most frequently during the lean period throughout the country. In some Indian states viz. Karnataka, Andhra Pradesh, and Chhattisgarh the PPR outbreaks in sheep and goats have declined after implementing the strategic mass vaccination control programme. The decreased number of outbreaks in the recent past as well as changes in the disease severity patterns and distribution might be due to the existence of institutional infrastructure, effectiveness of vaccine, timely vaccination and most importantly effective planning and implementation of the vaccination programme. In overall, fixed strategies may not work for all the states or region or the countries. However, in the mass vaccination in pulse polio model with two to three cycles of vaccination, with each cycle of covering entire population of sheep and goats initially, subsequently bi-annual vaccination covering the naïve young population in a predesignated stipulated period for 2 years, followed by entire population, will have a tremendous impact on the control of PPR outbreaks in sheep and goats and subsequent eradication. Vaccinating the migratory population at the check post or border regions of the states or inter-state border or in the place of entry or place of trade market of animal through transport from other states are to be targeted for mass vaccination as and when required. Sharing the experiences on the PPR vaccination strategies adopted by some of the successful states in India may provide directions for other Indian states or other countries of similar socio-economic and small ruminant rearing pattern to vaccinate and control PPR. 


\section{Epidemiology of Japanese Encephalitis in Animals: An Indian Perspective}

\section{Baldev R. Gulati}

Principal Scientist, ICAR-National Research Centre on Equines, Hisar, Haryana

Japanese encephalitis (JE) is an important and widespread mosquito-borne zoonotic viral infection of horses, pigs and humans. JEV causes reproductive disorders in pregnant sows and encephalitis in horses. JE is a disease of rural agricultural areas where vector mosquitoes proliferate in close association with pigs, wading birds, and ducks. The principal vector species is Culex tritaeniorhynchus, which is present in great density in rainy season in both tropical and temperate regions. This paper gives an overview of epidemiological factors associated with the spread of JE infection in animals, current diagnostic techniques and options for controlling JE infection in animals. JEV is a plus-sense, single-stranded RNA of $\sim 11 \mathrm{~kb}$ belonging to the genus Flavivirus in the family Flaviviridae. Based on E gene phylogenetic analysis, JEV strains have been classified into five genotypes $(\mathrm{G} 1-\mathrm{V})$. Although GIII is most widely distributed genotype in human population, GI is emerging predominantly in South Korea, Thailand and China and India. In animals, GIII is widely distributed in pigs from several countries, including India while GI from China, Cambodia, Korea \& Thailand. Equine JEV isolates from Japan, Hong Kong and Taiwan have been typed as GI, GII and GIII. We isolated JEV from a horse in India that belongs to GIII. A definitive diagnosis of JE depends on isolation or demonstration of virus in sick or dead animals. The routine laboratory diagnosis of JEV infection is based on culture and serology followed by identification by RT-PCR. Virus isolation is done by intra-cerebral inoculation of clinical specimens in suckling mice or passaging in Vero, BHK21, LLCMK2, C6/36. Molecular diagnostic methods for diagnosis include RT-PCR, fluorogenic (TaqMan) or SYBR greenbased real-time PCR and loop-mediated isothermal amplification (LAMP). Serological tests in animals include virus neutralization (VN), haemagglutination inhibition (HI), complement fixation (CF) and ELISA and IgMcapture ELISA. JEV is transmitted in a zoonotic cycle among mosquitoes and vertebrate-amplifying hosts, chiefly pigs and wading birds. Pigs serve as amplifying hosts because they develop viremia that remains high enough to infect mosquitoes for up to 4-14 days. Humans and horses are considered as the dead-end hosts as they have brief periods of viremia with low virus titres. The ardeid wading birds (heron, egrets) are considered the primary enzootic hosts of JEV and play important role in epizootic viral amplification. Mosquitoes that commonly transmit JEV are most heavily concentrated in rural, rice growing areas and feed most actively in the late afternoon and early evening. Although JEV has been isolated from over 30 species, paddy-breeding mosquitoes of the Culex vishnui subgroup, particularly $C x$. tritaeniorhynchus, are the major vectors of the virus. Till recently, JEV transmission has been exclusively described as being mosquito-mediated. However, recently it has been demonstrated that JEV can be transmitted between pigs in the absence of arthropod vectors. Pigs shed virus in oronasal secretions and are highly susceptible to oronasal infection. Further, virus persists in pig tonsils for at least 25 days. These findings could have a major impact on the ecology of JEV in temperate regions with short mosquito seasons. Persistence of viral infection under low environmental temperature has been demonstrated in insectivorous bats.

The ecology of Assam represents a classical example of reasons for upsurge of JE in human and animal population. JE occurred in sporadic outbreaks or epidemic forms in upper Assam since 1976, but has become endemic and highest number of human casesare reported from Assam since 2011. It may be attributed to frequent floods, increasing rice \& pig farming, and abundance of mosquitoes. We reported $22.99 \%$ of 335 pigs JEV sero-positive during 2015-2016. There was significantly higher JEV positivity in pigs from Sivasagar district $(31.31 \%)$ than in Kamrup Rural district $(11.51 \%)$. With the global warming, there has been increase in JE vector proliferation and longevity. The changing agricultural practices such as irrigation, increase in irrigated rice-production area has resulted in increase in JE outbreaks. Climate change is also influencing migration patterns of birds, which may result in introduction of JEV into new areas. In contrast to pigs, sporadic cases of JE occur in horses in India. Serosurveillance among equines in 13 different states of India revealed maximum JE positivity in Manipur followed by those in Gujarat, Madhya Pradesh and Uttar Pradesh. JEV has been isolated from a horse showing neurological signs of encephalitis from Haryana. Phylogenetic analysis of the full-length sequence indicated that the JEV strain isolated from horse in India belongs to genotype III. There are still no specific drugs available to treat JEV infection. Prevention of JEV is based on mosquito control, avoiding mosquito bites, pig and human immunization. Vector control is one of the most important aspects of the prevention of JE spread. Larvicides and insecticides help in controlling mosquitoes in paddy fields. Multiple types of JE vaccines are available for humans including mouse brain-derived inactivated, cell culture-derived live-attenuated, cell culture-derived killed-inactivated, and genetically engineered live-attenuated chimeric vaccines. Other experimental vaccines, including recombinant poxvirus vaccine, DNA-based vaccine are also under various stages of development for human use. JE in livestock is a notifiable OIElisted disease that could affect trade between countries. Vaccination is recommended for the control of disease in animals for reducing abortions in pigs or protecting high value race horses. In South Korea, a live attenuated JE vaccine (Anyang 300 strain, G3) has been used in swines for more than 30 years. This has proven highly effective at reducing incidence of disease in pigs. A formalin-inactivated vaccine derived from porcine kidney cells or chick embryo cells and liveattenuated vaccines derived from porcine kidney cells are used in swines or horses in several Asian countries. Vaccination of horses is effective in protecting against disease, particularly high value animals such as racehorses that travel to JE endemic regions. In India, no vaccination has been practiced for animals, including horses and swine. Since pigs are the amplifying host for the JEV, vaccination in pigs in the JEV-endemic areas will help in curtailing the source of JEV infection. Vaccinations of pigs will break the mosquito-pig-human transmission cycle and thus helping in control of JEV. Intensification and expansion of irrigated rice production systems over the past few years have contributed in increased JE incidence. Effective JE control is possible by means of effective surveillance systems and timely vaccination of persons or animals at risk. The animal diagnostic facilities for JE in India need to be strengthened for timely diagnosis and forecasting of the impending outbreaks. Newer diagnostic methods are needed for monitoring the spread of the disease in human, animal and vector population. There is no specific treatment for the disease. There is need for development of safe and effective vaccines for use in animals. Strategies for the reduction of virus burden in ecology by controlling vector population and relocating pig populations away from human habitation may be considered for effective global control of JE.

\section{Highly pathogenic avian influenza - an update on genetic diversity, geographical spread and its control and containment}

\section{Tosh, S. Nagarajan, Manoj Kumar}

ICAR-National Institute of High Security Animal Diseases, Anand Nagar, Bhopal - 462 022, India

H5 and H7 Highly Pathogenic (HP) Avian Influenza (AI) viruses represent a threat to animal health, human health and economy. Currently circulating H5 HPAI virus, emerged in 1996 in China, is 
evolving and continuing its spread by infecting poultry, other birds and occasional human infection. Following spread of H5 virus in other Asian countries, it had spread to Europe, Africa and North America, and wild birds are considered as the cause of intercontinental spread. The virus has evolved into multiple genetic clades (namely 0-9), and recently it has undergone reassortment with other low pathogenic AI (LPAI) viruses to generate novel H5N2, H5N3, $\mathrm{H} 5 \mathrm{~N} 6$ and $\mathrm{H} 5 \mathrm{~N} 8$ subtypes. The $\mathrm{H} 5$ virus is endemic in Bangladesh, China, Egypt, Indonesia and Vietnam. In 2013, a novel H7N9 LPAI virus emerged in live poultry markets and is responsible for multiple epidemic waves of human infections in China. In 2017, the H7N9 LPAI virus mutated to HPAI virus in poultry and is spreading westward in China. Two major genetic lineages of H7N9 virus (Pearl River Delta and Yangtze River Delta) has been identified. Besides $\mathrm{H} 7 \mathrm{~N} 9$, other $\mathrm{H} 7$ subtypes circulating in recent years are $\mathrm{H} 7 \mathrm{~N} 2$ (Australia), H7N3 (Mexico) and H7N7 (UK). Majority of the countries are following detect and culling policy for control and containment of HPAI. However, vaccination is advised as one of the components of a comprehensive control plan.

India, since its first report in 2006, has reported 159 H5 HPAI outbreaks (H5N1-126 and H5N8- 33) in 22 States/Union Territories till March, 2018. Genetic analysis of the viruses indicated multiple genetic clades (H5N1: 2.2, 2.2.1, 2.3.2.1a and 2.3.2.1c, and H5N8: 2.3.4.4) of the viruses indicating multiple introductions of the viruses. Currently, India is following "detect and culling" policy for control of HPAI. However, transboundary nature of the virus warrants international collaboration with neighboring countries for effective control and containment of HPAI.

\section{Advances in diagnosis and management of economically important endemic and emerging viral diseases of pigs}

\section{Dr. Dilip Kumar Sarma}

Email ID for Correspondence: dksarma1956@gmail.com Ex.Director, ICAR-NRC on Pig and Professor; Department of Microbiology, Faculty of Veterinary Science, Assam Agricultural University, Khanapara, Guwahati-781022

Pig rearing is one of the fastest growing livestock sector worldwide as it is an important source of rural livelihood and valuable source of animal protein. Pigs are also facing myriads of problems particularly the viral diseases, which have the capacity to decimate entire herds. Pigs are also regarded as mixing vessel, which gives opportunity to evolve new virus strains capable to cause pigs and human health problems. Despite huge opportunity and potentiality of the piggery the growth of this sector is slow in India. A number of endemic and emerging viral diseases like classical swine fever (CSF), foot-andmouth disease (FMD), porcine circovirus type 2 infection, porcine parvovirus infection, swine pox and porcine reproductive and respiratory syndrome (PRRS) etc. are causing constant threat to the pig population of India. Besides, with the increasing trend of human population, more demand for pork, globalisation, climate change and porous international borders particularly in the North Eastern Region may lead to emergence of newer diseases for which newer tools will be important to predict, detect and mitigate in future. In order to reduce the risk associated with the endemic and emerging viral diseases more robust disease surveillance, rapid and sensitive diagnostic tests, quality vaccines and improved management are essential along with strengthening of veterinary services and infrastructure and educating the pig rearers about the diseases and importance of better management.

Conventional diagnostic tests are widely used, but most of the tests are time consuming, less sensitive and laborious. It is also difficult to accurately and confidently predict viral infection status based on the clinical symptoms. During the last decade there is enormous progress in the development of molecular techniques which have not only accelerated disease diagnosis, but also facilitated to differentiate virus strains and pinpoint the source of infections. Several PCR assays have been developed for rapid detection of the pathogens. More than one of these pathogens can be detected simultaneously by the use of multiplex PCR. Reverse transcriptase loop mediated isothermal amplification (RT-LAMP) along with its modifications has been reported for detection of the viruses in less time with limited facility. Despite the progress, newer diagnostic tests developed in the country need validation, inter laboratory comparison or external quality assurance and harmonisation. Besides, there is a need to work with one world,one health concept for managing the economically important diseases of pigs and to develop user friendly, cost effective and field based diagnostic for rapid and specific detection of the diseases in India. In the present paper advances in diagnosis more particularly the molecular tests developed for the diseases of pigs in India along the management of the diseases will be discussed.

\section{RNA viruses: greatest global threat and one health solutions}

\section{Prasad Minakshi ${ }^{\mathbf{a}^{*} \mathrm{~b}}$, Basanti Brar ${ }^{1 \mathrm{a}}$, Upendra P Lambe ${ }^{1 \mathbf{a}}$, Koushlesh Ranjan $^{4 a}$, Gaya Prasad ${ }^{4 a}$, Harimohan ${ }^{5 a}$, Jinu Manoj ${ }^{6 a}$}

Email ID for Correspondence: minakshi.abt@gmail.com

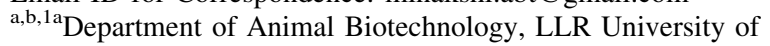
Veterinary and Animal Sciences, Hisar-125 004, Haryana, India; ${ }^{2 \mathrm{a}}$ CIRB, Hisar; ${ }^{3 \mathrm{a}}$ Department of Veterinary Physiology, COVAS, KVASU, Pookode, Wayanad- 673576, Kerala, India; ${ }^{4 a}$ SVP University of Agriculture and Technology, Meerut, India; ${ }^{5 a}$ IPVS, LUVAS, Haryana, India; ${ }^{6 a}$ RVDEC Mahendergarh, LUVAS, Haryana, India

RNA viruses can be divided into various classes on the basis of their genome polarity: positive-strand RNA viruses (PSVs) and negativestrand RNA viruses (NSVs), linear or segmented based on genome arrangements. RNA viruses reproduce less accurately. They usually are deficient in proofreading and more prone to mutation rates of any other organism on planet earth. These mutation rates mean that a large complex genome is not possible because their high error rates would cause offspring requiring a large gene set to be nonfunctional. RNA viruses, therefore, have small genomes and lesser genes. The benefit of such a high error rate is that RNA viruses are able of rapidly outmaneuvering the host immune system. The approach of RNA viruses is usually fast reproduction and moving on to a new host. Because they have less complex relationships with their hosts, RNA viruses are much more capable of moving to new host species. The ability to move to new hosts reduces the selective pressure to not harm the host, and many RNA viruses are more pathogenic. In the present globalized world with overflowing travels and trades, human health has been increasingly threatened due to incidences of emerging and re-emerging infectious viral diseases. The most important sources of novel human pathogens are mainly farm mammals, poultry, to some extent wild animals and arthropods. It is estimated that zoonosis constitute about $60 \%$ of the 'known', and up to $75 \%$ of 'emerging' new human infections. Over the past several decades, sporadic and often isolated outbreaks of emerging human diseases have led to the discovery of a diverse array of novel, highly pathogenic viruses not limited to Filoviridae, Arenaviridae, Annuloviridae, Picobirnaviridae, Bunyaviridae, Paramyxoviridae, Coronaviridae and Flaviviridae families. Emergences as well as reemergence of several viral infections namely Nipahvirus, Hantaviruses, Chikungunya, Human Enterovirus-71, Influenza, Chandipura, Crimean 
Congo, SARS, Picobirnavirus, Coronavirus, Buffalopox, Dengue and Japanese Encephalitis viruses has-been reported from developing countries including India. The transmission of agents depends upon long time interactions between people and domestic animals or nearby wildlife. The key to understand the emergence and re-emergence viruses is to know the 'host-pathogen-environment' relationship in virus evolution. It is worth to mention that increasing number of cases reported globally is alarming, indicating humans as source of transmission of infectious viruses to domestic and wild animals. Recent examples include influenza A, astrovirus, enterovirus, human adenovirus, human metapneumovirus, human corona, hepatitis A, norovirus, rotavirus etc. The most affected animals are wild followed by livestock and companion animals. Recent isolation and genetic analysis of human $\mathrm{H} 1 \mathrm{~N} 1$ and $\mathrm{H} 3 \mathrm{~N} 2$ influenza viruses from pigs in China, human rotavirus from sheep and buffalo from India and developed countries provide strong evidence of reverse zoonoses where transmission of viral agents is not restricted to developing countries, indicating a worldwide disease threat. Various contributing factors namely rapid movements of humans and animals, overall increase in industrial animal production, and conflict of habitats of humans and wild animals are adding to opportunities for humans to cause reverse zoonoses.

Zoonotic infectious diseases have been an important concern to humankind for more than 10,000 years. Today, approximately $75 \%$ of newly emerging infectious diseases (EIDs) is zoonoses that result from various ecologic, socioeconomic, genetic, anthropogenic and climatic factors. These interconnected driving forces make it complicated to envisage and to prevent zoonotic EIDs. While considerable improvements in various areas of environmental and medical surveillance, clinical analytical methods and medical practices have been achieved in current years, EIDs remain a foremost global concern particularly in less developed regions. The current Ebola epidemic in West Africa is a tremendous harsh reminder of the role animal reservoirs play in public health and reinforces the vital need for globally operationalizing a 'One Health approach'. The complex nature of zoonotic diseases and the limited resources in developing countries are a reminder for implementation of Global One Health in low-resource settings is essential. The Veterinary Public Health and Biotechnology Global Consortium launched the International Congress on Pathogens at the Human-Animal Interface (ICOPHAI) in order to tackle significant challenges and desires for capacity building. In One Health implementation, four key capacity-building step needs: establishment of sufficient science-based risk management policies, skilled-personnel capacity building, accredited veterinary and public health diagnostic laboratories with a shared database, and improved use of existing natural resources and implementation. The future scientific research efforts must be aimed in areas of zonotic and zooanthroponosis, for better understanding and transmission of various viral agents amongst various hosts resulting in accurate diagnosis and control measures. Multidisciplinary approaches such as 'One Health One World' is the need of the hour to mitigate these problems.

\section{Emergence of novel bovine viral diarrhoea viruses and other ruminant pestiviruses: Implications on BVD control}

\section{Dr. Niranjan Mishra}

Email ID for Correspondence: nmishra@hsadl.nic.in Principal Scientist, ICAR-National Institute of High Security Animal Diseases, Anand Nagar, Bhopal, Madhya Pradesh, 462022, India

Bovine viral diarrhea virus (BVDV) is an important pathogen of cattle with a global distribution and causes major economic losses due to a variety of disease syndromes including the highly fatal mucosal disease. BVD is caused by BVDV-1, BVDV-2 and BVDV-3 with considerable genetic and antigenic heterogeneity of BVDV strains. BVDVs belong to the Pestivirus genus within the Flaviviridae family that also comprises the genera Flavivirus, Hepacivirus and Pegivirus. Significant differences exist in the virulence of BVDV strains. BVDVs are highly successful to persist and spread in their host populations due to their unique ability to produce persistent infection through evasion of adaptive immune response and innate immune response. Recent advances in diagnostic methods, nucleotide sequencing and computer-assisted phylogenetic analyses have so.far identified twenty-one BVDV-1 subtypes (BVDV-1a to -1u), four BVDV-2 subtypes (BVDV-2a to -2d) and four BVDV-3 subtypes (BVDV-3a to -3d). Successful BVD control is highly dependent on removal of PI animals, movement controls for infected herds, strict biosecurity, surveillance and vaccination as an additional tool. Although vaccination has reduced the clinical outcomes and spread of $\mathrm{BVD}$, over the years vaccination alone has not resulted in the elimination of BVDV related clinical disease or a significant reduction in BVDV losses.

Emergence of novel BVD viruses, such as mixed triple highly virulent BVDV-2c has been reported recently with high mortalities in cattle in Germany and Netherlands, where coexistence of 3 distinct genomic variants was evident with unusual mutations in two variants. Additionally, co-circulation of novel and highly divergent BVDV-3 viruses in cattle has recently been reported from India and BVDV-3 associated mucosal disease cases in some countries are on the rise. Although BVDV spread from wild ruminants to cattle has a low to moderate risk, risk is high for BVDV transmission from sheep and goats. Emergence of other ruminant pestiviruses include Tunisian sheep pestivirus, Turkish sheep pestivirus and Pronghorn antelope pestivirus. Furthermore, emergence of border disease virus (BDV) in wild ruminants and increasing reports of natural transmission of BDV from sheep and goats to cattle leading to generation of PI cattle and further cattle to cattle transmission pose additional challenges for control in BVD free countries. Virological screening for identification and elimination of PI animals has been the hallmark for BVD control. However, acutely infected animals with highly virulent BVDV strains have recently been observed as a high risk of spread and should not be ignored. The present lecture will focus mainly on emergence of novel BVDV strains and other ruminant pestiviruses, and its implications on BVD control at global level so that adequate changes in monitoring and control strategies can be formulated and implemented.

\section{Emergence of transboundary viral diseases of pigs in North Eastern States: a threat for India's main land}

\section{N. N. Barman, A Sen, T Dutta, D. Hemadri, S. S. Patil, Suresh, B. C. Bera}

Email ID for Correspondence: nnbarman@gmail.com Department of Microbiology, College of Veterinary Science, Assam Agricultural University, Khanapara, Guwahati, Assam- 781022, India

The north eastern region of India shares highly porous and sensitive frontiers with China, Myanmar, Bangladesh and Bhutan. Again, rapid globalization opens frequent international movements of men/material/animals, including vector population and bears an ever increasing threat of incursions of exotic, trans-boundary diseases crossing the borders to India's main land through this North East corridor. A retrospective as well as prospective monitoring of emerging viral diseases of pigs is being carried out under DBT sponsored project involving three core NE laboratories, four National Labs and eight directorates of animal husbandry and veterinary in NER. Epidemiological data, various clinical samples and tissue samples of pigs were 
collected from eight states of NER. To generate disease incidence as well as sero-prevalence data of various infectious diseases of pigs, meta-analysis was conducted using the $\mathrm{R}$ Open source scripting software 3.4.3. Various molecular tools were used to confirm classical swine fever (CSF), porcine reproductive and respiratory syndrome (PRRS), porcine circovirus-associated disease (PCVAD), swine influenza (SI), torque tenosus virus (TTSuV) disease and swine pox diseases. Sequencing was done to understand molecular epidemiology of all emerging swine viruses. In appropriate conditions, isolation of viruses was done. All sera samples were subjected for prevalence of antibody in unvaccinated population using in-house optimized i-ELISA/or standard commercial kits.

Classical swine fever is endemic in NER. However, new genogroups are emerging from neighbouring countries. Out of reported occurrence of CSF, annual outbreaks were recorded as 24.46. Seroprevalence of CSFV specific antibody indicated $31.00 \%$ in meta analysis. Further, CSFV genogroups 1.1, 1.2, 2.1 and 2.2 were circulating in NER. Emergence of porcine circovirus-associated disease (PCVAD) of pigs caused by Circovirus 2 was confirmed. Sofar, 124 incidences were found positive for Circovirus-2 and also authenticated by gene sequence analysis. Serological survey showed that $43.00 \%$ pigs was positive for porcine circovirus. Again, porcine reproductive and respiratory syndrome (PRRS) was identified as new emerging pathogen of pig in the state Meghalaya followed by in Mizoram. The incidence was recorded as $13.03 \%$ in these two states. Authenticated sero-prevalence of PRRS was $2.00 \%$. All the samples were screened by RT-PCR assay for detection of ORF7 and ORF4 genes and sequence analysis showed close grouping with PRRSV isolates of genotype 2 (North American type) from China. Swine influenza virus was detected in pigs of NER. Out of 69 nasal swabs from pigs, 13 samples showed positive amplification of matrix gene of influenza A virus. Sequencing of PCR amplicons (244 bp) revealed 90-99.9\% identity to the influenza A viruses. In these study, torque tenosus virus (TTSuV) a small non-enveloped virus, containing single-stranded, negative sense circular DNA was identified in limited NE states. The virus commonly acts as cofactor with PCV and PRRS. Out of 51 samples 4 and 8 samples were found positive for TTSuV1 and TTSuV2, respectively. Along with climate change swine pox has been re-emerged in North Eastern States. A total of 9 outbreaks were confirmed as swine pox. On sequence analysis, SWPV isolates shared maximum identity (99-100\%) with the previously reported SWPV isolate from India (AVA121) as well as from other countries. Swine pox virus showed a distinction from other poxviruses. Isolation of CSFV, PCVADV, SIV, SWPV was done successfully. Confirmation of these emerging viral pathogens of pigs circulation in NER is a valuable information and warrants preparedness to curb spreading of these viruses to India's mainland.

\section{Role of Next Generation Sequencing in discovery of neglected viral pathogens of family Reoviridae}

\author{
Yadav $\mathbf{P}^{1}$, Shete $\mathbf{A}^{2^{*}}$ and Mourya $\mathbf{D}^{3}$ \\ Email ID for Correspondence: anitaaich2008@gmail.com, \\ shete.aa@niv.co.in \\ ${ }^{1}$ Scientist E and Head of department, Maximum Containment \\ Facility,ICMR- NIV Pune; ${ }^{2}$ Scientist D, Maximum Containment \\ Facility ICMR- NIV Pune; ${ }^{3}$ Director, Indian Council of Medical \\ Research, National Institute of Virology, Pune
}

Detection of novel pathogenic viruses in the clinical samples has special significance in the public health owing to the fact that many recent disease outbreaks have been reported from the novel pathogens. The Next-Generation Sequencing (NGS) has revolutionized the genomic approach for detection of the viruses. The unbiased approach of sequencing implied in NGS has a major role in the detection of unknown or uncharacterized pathogens. Total RNA extractions was performed using supernatants and pellets obtained from cell culture and mice brain suspensions. Libraries were prepared using the TruSeq LT Stranded mRNA Library Preparation Kit. The denatured libraries were loaded onto Illumina Miniseq mid-output 150 reagent cartridges. The resulting fastq files analyzed using CLC Genomic Workbench. We have characterized four previously un identified reoviruses from India. Wad Medani virus (WMV), isolated in 1954 from a Hyalomma marginatum ticks pool from Pune, Kammavampattai virus (KVPTV) isolated in 1963, from Sturnia pagodarum, Brahmani Myna bird from Tamil Nadu. One tick isolate was identified as Kundal virus a member of the coltivirus genus. Equine encephalosis virus (EEV), a reovirus, isolated from a dead horse in 2008, first time from India. Identifications of previously uncharacterized isolate lead to design assays for these viruses. Molecular and serological assays developed can be used for further studies. Diagnosis of the pathogenic viruses using NGS technologies can play a vital task in dealing with the global viral epidemics. This in turns can help in preparing for the emerging viral infections which can emerge as a major public health challenge.

\section{Expression of VP2 protein of bluetongue virus serotype- 1 in baculovirus system and its potential use as a serotype specific diagnostic antigen}

\author{
Chand $\mathbf{K}^{1}$, Sharma $\mathbf{P}^{2}$, Biswas $\mathbf{S ~ K}^{3}$, Lohumi $\mathrm{A}^{4}$ \\ and Ramakrishnan M.A
}

Email ID for Correspondence: virusshield@gmail.com Division of Virology, ICAR-Indian Veterinary Research Institute, Mukteswar Campus, Nainital -263 138, Uttarakhand, India

Bluetongue (BT) is an arthropod-borne disease of domestic and wild ruminants, caused by bluetongue virus (BTV). At present, worldwide 27 distinct BTV serotypes have been recognized. The BTV genome consists of ten segmented (dsRNA) which codes for seven structural and four non-structural proteins. BT is enzootic in India and amongst the 23 serotypes reported to be circulated in the country, BTV-1 is one of the major concerns. Detection of serotypes is necessary for taking up effective control measures including vaccination. VP2 is the most variable of the BTV proteins and is the major serotype determinant. Therefore, the experiment was designed to produce recombinant VP2 protein and evaluate its diagnostic potential for detection of type-specific antibody against BTV-1 serotype. The truncated N-terminal BTV (1)-VP2 (aa636), gene of BTV serotype-1 was amplified, cloned and expressed in baculovirus expression system. For the recombinant baculovirus expression in Sf21 cells, the VP2 gene, cloned into an appropriate baculovirus expression vector $\left(\mathrm{pFastBac}^{\mathrm{TM}}\right)$ and transposed into the bacmid DNA of DH10Bac ${ }^{\mathrm{TM}}$. The recombinant bacmids are then isolated and used to transfect Sf21 cells. The analysis of the expressed recombinant BTV (1)-VP2 proteins on SDS polyacrylamide gel revealed the protein corresponding to the theoretical molecular weights of BTV(1)-VP2(aa636). By separating the soluble and insoluble proteins of the Sf21 cell lysate, also revealed that the recombinant BTV (1)-VP2 proteins were soluble since they were only found in the supernatant fraction. Further, the diagnostic potential of the recombinant VP2 was evaluated by indirect ELISA using HIS against BTV-1 and serum sample of sheep and goat origin. The recombinant VP2 antigen based i-ELISA will be 
a good choice for detection of type specific antibody against BTV-1 in the sero-epidemiological studies. The type-specific i-ELISA may be a suitable alternative to the cumbersome process of conventional serum neutralization assay.

\section{Molecular epidemiology and genetic analysis of coronaviruses from large and small ruminants}

\author{
Sircar $\mathrm{S}^{1^{*}}$, Malik Y. $\mathrm{S}^{2}$, Bhat $\mathrm{S}^{3}$, Agarwal R. $\mathrm{K}^{4}$, Abhisek ${ }^{5}$, Sankar \\ $\mathrm{M}^{6}$ and Muthuchelvan $\mathrm{D}^{7}$
}

Email ID for Correspondence: shubhankar.sircar@gmail.com $1,2,3,4,5$ ICAR - Indian Veterinary Research Institute, Izatnagar, Bareilly, Uttar Pradesh-243122, India; ${ }^{6,7}$ ICAR - Indian Veterinary Research Institute, Mukteswar, Nainital, Uttarakhand-263138, India

Coronavirus $(\mathrm{CoV})$ is a significant cause of diarrhea in calves and goat kids, winter dysentery in adult cattle, and also respiratory infections in calves. Despite its global connotation, epidemiological data pertaining to $\mathrm{CoV}$ infection in animals from India is occasional. Therefore, a survey was conducted to detect $\mathrm{CoV}$ infection in dairy calves and goat kids and analyze the virus isolates genetically. From April 2017 to March 2018, 151 fecal samples from cattle $(n=65)$, buffalo $(n=22)$ and caprine $(n=64)$ were screened for CoV infection, respectively using RT-PCR based detection system targeting the most consensus region from nucleocapsid gene $(\mathrm{N})$ segment for the $\mathrm{CoV}$. Amplicons of $668 \mathrm{bp}$ were cloned in pDrive vector and sequenced from both sides followed by sequence and phylogenetic analysis. RT-PCR based findings confirmed the positivity of $\mathrm{CoV}$ in $16.92 \%(11 / 65)$ of cattle, $22.73 \%(5 / 22)$ of Buffalo and 9.38\% (6/64) of caprine samples, respectively. Overall percent positivity for the $\mathrm{CoV}$ infection was $14.56 \%$ (22/151). Cloning and sequencing of representative $\mathrm{CoV}$ positive samples from cattle for $\mathrm{N}$ gene and replicase gene (ORF1b) revealed that the study strains are quite distinct and remain outside the major clade comprising of different species of $\mathrm{CoV}$ in the phylogram. The findings of study affirms that $\mathrm{CoV}$ infection exists in large and small ruminant population in the country and additionally the strains of $\mathrm{CoVs}$ circulating in Indian livestock population are genetically diverse. More surveillance studies are needed to know the exact status of $\mathrm{CoV}$ infections in farm animals.
CRISPR/Cas9- Mediated Genomic Deletion of the HERC-5 gene in B95a (Marmoset B-lymphocyte cells) to limit the PPRV replication

Shikha Saxena ${ }^{a}$, Amit Ranjan Sahua, Aruna Pandeya, Sajad Ahmad Wani $^{\mathrm{a}}$, Piyali Mondal ${ }^{\mathrm{a}}$, Raja Ishaq Nabi Khan ${ }^{\mathrm{a}}$, Waseem Akram Malla $^{a}$, Sonalika Mahajan ${ }^{\mathrm{a}}$, Bina Mishrab, Ashok K Tiwari ${ }^{c}$, Bishnu Prasad Mishra a , Raj Kumar Singh ${ }^{\text {a }}$, Deepak Kumar $^{\mathrm{a}^{*}}$, Ravi Kumar Gandham ${ }^{\mathrm{d}^{*}}$

Email ID for Correspondence: saxena.shikha96@gmail.com ${ }^{\mathrm{a}}$ Division of Veterinary Biotechnology, ICAR-IVRI, Izatnagar, Bareilly, UP-243122; ${ }^{\mathrm{b}}$ Division of Biological Products, ICAR-IVRI, Izatnagar, Bareilly, UP-243122; ${ }^{\mathrm{c}}$ Division Standardization, ICARIVRI, Izatnagar, Bareilly, UP-243122; ${ }^{\mathrm{d}}$ National Institute of Animal Biotechnology, Hyderabad, AP- 500075

Peste des petits ruminants (PPR), a highly contagious disease affecting goats and sheep. Sungri/96 vaccine strain is widely used for mass vaccination programs in India. Among the differentially expressed highly connected genes in goats and sheep, HERC-5 was found to be highly upregulated antiviral gene, identified previously by transcriptome analysis. B95a cells express high level of SLAM and extensively used for the infection of PPRV. In this study the role of the HERC- 5 gene in isgylation has been studied by knockout with CRISPR-cas9 in B95a cells for the PPRV replication. In this study to knockout the HERC-5 gene by CRISPR/cas9, three gRNA (guide RNA) were designed by four different softwares. Three common gRNA in all the softwares were chosen for the study. The activity of the gRNA was checked by in vitro cleavage assay. One gRNA was found to be best among three and cloned in GeneArt CRISPR.Ofp vector. The cloning was confirmed by PCR, and Sanger sequencing. The (GeneArtCRISPR.HERC5) was transfected in B95a cells to study the gene knockout and PPRV replication. Among the three gRNA for the HERC-5 gene the one in exon 1 was found to be the best by in vitro cleavage assay. The first gRNA1 of HERC- 5 gene was cloned in GeneArtCRISPR.Ofp and the genomic DNA of the cloned product was analysed by sequencing. By transfection of the HERC5 clone, the OFP expression was studied by FACS. The gene editing efficiency in B95a cells was confirmed cleavage assay and sequencing. After gene editing the B95a cells studied for PPRV replication by titration and Real time PCR. The study demonstrates the role of HERC5 gene as an antiviral agent. Our findings contribute to a growing body of evidence suggesting that HERC5 is a novel host restriction factor.

Publisher's Note Springer Nature remains neutral with regard to jurisdictional claims in published maps and institutional affiliations. 